\title{
Parameter Space Mapping for Blood Oxygenation Measurement with Low Field NMR
}

\author{
by \\ Dion Gary Thomas
}

\author{
A thesis \\ submitted to the Victoria University of Wellington \\ in fulfilment of the \\ requirements for the degree of \\ Master of Science \\ in Physics.
}

Victoria University of Wellington

2018 



\begin{abstract}
Blood oxygenation is a critical physiological parameter for patient health. The clinical importance of this parameter means that measurement of blood oxygenation is a routine part of care. Magnetic resonance provides a way to measure blood oxygenation through the paramagnetic effect of deoxy-haemoglobin, which decreases the $T_{2}$ relaxation time of blood. This effect has been well characterised at high fields ( $>1.5 \mathrm{~T}$ ) for use in Magnetic Resonance Imaging, and it is a contributing factor to the Blood Oxygenation Level Dependent contrast used in functional MRI. However there are relatively few studies of this effect at low magnetic fields, and these have only looked at extreme levels of oxygenation/deoxygenation. To study this effect for potential application in a low-field device, we measured this effect to determine how factors such as oxygenation, field strength and CPMG echo time affect the $T_{2}$ of blood.

A continuous flow circuit, similar to a cardiopulmonary bypass circuit, was used to control parameters such as oxygen saturation and temperature, before the blood sample flowed into a variable field magnet (set at fields between 5-40 $\mathrm{MHz}$ ), where a series of CPMG experiments with echo times ranging from $1 \mathrm{~ms}$ to $20 \mathrm{~ms}$ were performed to measure the $T_{2}$. Additionally, the oxygen saturation was continually monitored by an optical sensor, for comparison with the $T_{2}$ changes. This allowed us to test the sensitivity of this effect at low fields.

These results show that at low fields, the $T_{2}$ relaxation change still follows the trends shown in the literature, with a dependence on $B_{0}$ squared, and on the fraction of deoxyhaemoglobin squared. Additionally, these results were also compared with two theoretical models for the dependence on echo time, which have previously been tested at high fields: the Luz-Meiboom equation, and the Jensen and Chandra model. Both models gave good agreement with the data measured at low fields. These experiments show that the $T_{2}$ changes in blood due to oxygenation are still visible at low field, and that this technique should be feasible in a low field device.
\end{abstract}




\section{Acknowledgments}

Firstly I need to thank my supervisors: Sergei Obruchkov, Shieak Tzeng and Petrik Galvosas for their excellent supervision, and for giving me the opportunity to pursue this research. In particular I would like to thank them for their encouragement when the baby-MRI decided to choke a month before I was due to give a talk about results collected on it. I would like to think that the panic and stress that caused was worth it.

Another big thanks goes to the other members and friends of the VUW NMR group over the last 2 years. Thank you for making this such a fun place to work, and for all of your help and friendship. To my other past supervisors, Marcel and Tim, thank you for introducing me to NMR and for letting me spend my honours year here.

Next, I would like to thank my fantastic friends and flatmates. Thanks to them for putting up with my work stories, and for helping to keep me sane. Of course, I must also thank Wilbur, who is somehow always excited to see me get home.

I would not have been able to complete this work without the care and support I have received from my family. Thanks to my Mum and Dad for their encouragement, advice and support, and for treating me to weekend brunches. And to my brother Adrian for his motivated assistance in acquiring research materials. Thanks also to my family in the Philippines, especially my Lola, for their support and prayers.

I gratefully acknowledge funding support from the New Zealand Ministry of Business, Innovation and Employment, and the Australian and New Zealand Society for Magnetic Resonance for a travel grant to present this work at the ANZMAG conference.

I look forward to more experimenting in the future 


\section{Contents}

1 Introduction 1

2 Background $\quad 5$

2.1 Nuclear Magnetic Resonance ... . . . . . . . . . . . . 5

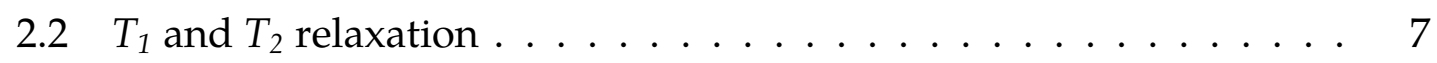

2.3 Spin echoes and CPMG . . . . . . . . . . . . . . 9

2.4 PGSE experiments . . . . . . . . . . . . . . . . 11

2.5 Blood and oxygen saturation . . . . . . . . . . . . . . 13

2.6 Oxy- and deoxy-haemoglobin . . . . . . . . . . . . . . . . . . . . . . . . . . . . . . . . . 14

$2.7 \mathrm{sO}_{2}$ measurement . . . . . . . . . . . . . . . . 16

$2.8 T_{2}$ changes due to oxygenation in blood . . . . . . . . . . . . 17

2.9 Literature on NMR measurement of oxygen saturation . . . . . . 19

3 Experimental Setups 23

3.1 Stopped flow experimental setup . . . . . . . . . . . . . 23

3.1 .1 Flow circuit and pump ................ 23

3.1 .2 Oxygenator and Gas Mixer . . . . . . . . . . . . . . 25

3.1.3 Blood collection . . . . . . . . . . . . . 25

3.1 .4 Oxygenation measurement ............. 26

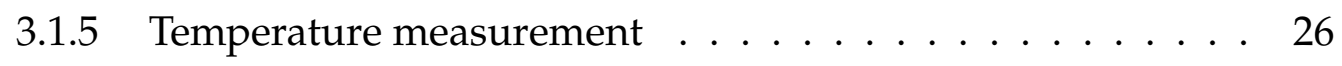

3.1 .6 NMR setup . . . . . . . . . . . . . . . . . . . . . . . . . . . . . . 27

3.2 Continuous flow setup . . . . . . . . . . . . . . . . . . 29

3.2.1 Flow Circuit Changes . . . . . . . . . . . . . . . . . . 29

3.2.2 Baby-MRI magnet . . . . . . . . . . . . . 30

3.2.3 Coil Assemblies and Electronics . . . . . . . . . . . . . 31

3.2.4 Flow measurement by NMR ... . . . . . . . . . . . . . 33

3.2.5 Optical $\mathrm{sO}_{2}$ measurement ............... 33

4 Stopped flow $\mathrm{sO}_{2}$ measurements 37

4.1 Experimental Protocol . . . . . . . . . . . . . . 37 


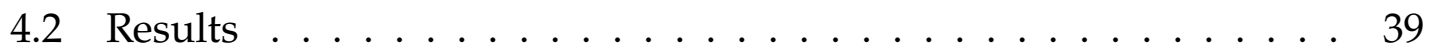

4.2.1 Spinsolve results ................. 39

4.2 .2 Halbach results . . . . . . . . . . . . . . . . . 40

4.2 .3 MOLE results . . . . . . . . . . . . . . . . 42

4.2 .4 Field comparison . . . . . . . . . . . . . . 44

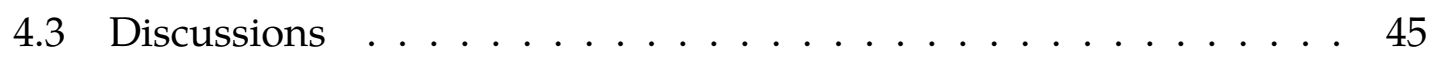

4.4 Summary . . . . . . . . . . . . . . . . 46

5 Continuous measurements of $\mathrm{T}_{2}$ and $\mathrm{sO}_{2} \quad 49$

5.1 Experimental Protocol . . . . . . . . . . . . . . . . . . . . . . 49

5.1 .1 NMR Experiments .................. 50

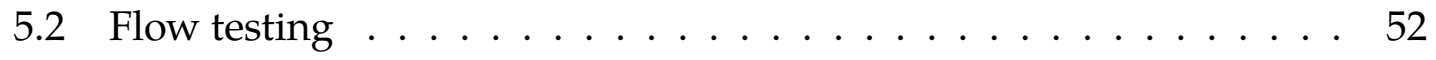

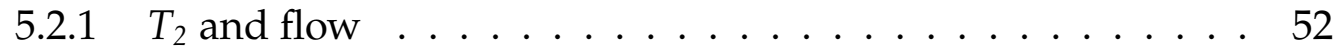

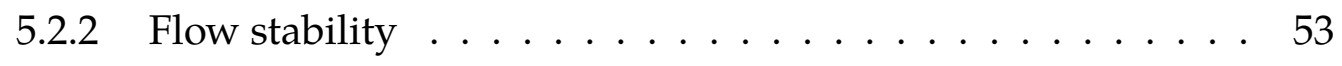

5.3 Blood results . . . . . . . . . . . . . . . 55

$5.3 .140 \mathrm{MHz} \ldots \ldots \ldots \ldots \ldots$

$5.3 .220 \mathrm{MHz} \ldots \ldots \ldots \ldots \ldots$

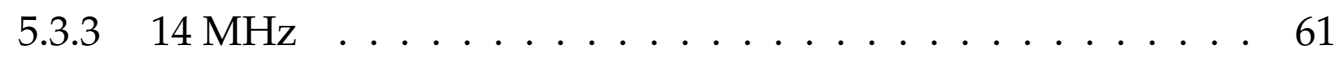

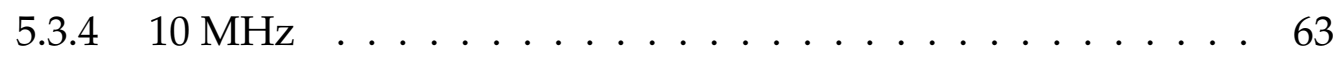

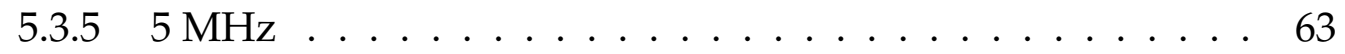

5.3 .6 Dependence on $\mathrm{sO}_{2} \ldots \ldots \ldots \ldots$

5.3 .7 Dependence on Field . . . . . . . . . . . . . . . . 67

5.4 Discussion . . . . . . . . . . . . . . . 70

5.4.1 Confounding factors . . . . . . . . . . . 71

5.4 .2 Comparison with literature . . . . . . . . . . 73

5.4.3 Background $T_{2}$ decrease: Haemolysis . . . . . . . . . 75

5.4.4 Oxygen saturation measurement from $T_{2} \ldots \ldots$

5.5 Summary . . . . . . . . . . . . . . . . . 80

6 Models for $T_{2}$ relaxation enhancement 81

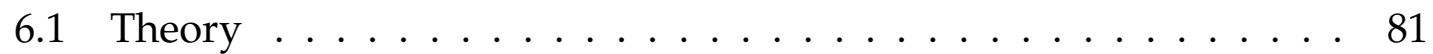

6.2 Experimental Setup . . . . . . . . . . . . . . . . 83

6.3 Results ............................. 85

6.3.1 $T_{2}$ Measurement ................ 85

6.3 .2 Model fitting . . . . . . . . . . . . . . 86

6.4 Discussion . . . . . . . . . . . . . . . . . 90

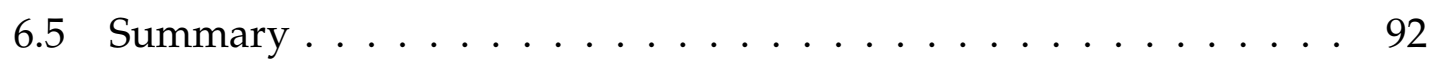


CONTENTS

7 Conclusions and Future Work 


\section{List of Figures}

2.1 Effect of the $B_{1}$ field $\ldots \ldots \ldots \ldots \ldots \ldots \ldots \ldots \ldots$

2.2 Inversion recovery experiment $\ldots \ldots \ldots \ldots \ldots$

2.3 Transverse relaxation due to an inhomogeneous field . . . . . . . 9

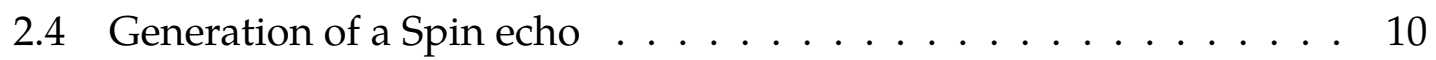

2.5 CPMG pulse sequence . . . . . . . . . . . . . . . 11

2.6 Pulsed Gradient Spin Echo (PGSE) experiment . . . . . . . . . . . 12

2.7 Illustration of red blood cells . . . . . . . . . . . . . . . . . 14

2.8 Graph showing relationship between $\mathrm{pO}_{2}$ and $\mathrm{sO}_{2}$ in blood . . . . 16

3.1 Schematic of the Stopped flow setup . . . . . . . . . . . . . . 24

3.2 Magnets used in stopped flow experiments . . . . . . . . . . 27

3.3 Schematic of the continuous flow circuit . . . . . . . . . . . 30

3.4 Variable field baby-MRI magnet . . . . . . . . . . . . . . . . 31

3.5 Probe and Tuning and Matching equipment . . . . . . . . . . 32

3.6 Optical $\mathrm{sO}_{2}$ sensor . . . . . . . . . . . . . . . . . . . . . . 34

3.7 Calibration data for optical $\mathrm{sO}_{2}$ sensor $\ldots \ldots \ldots \ldots \ldots$

4.1 Experimental protocol for stopped flow experiments . . . . . . . . 38

4.2 Experimental setup for stopped flow experiments . . . . . . . . . 39

4.3 Examples of CPMG decays measured on the Spinsolve for oxygenated and deoxygenated blood . . . . . . . . . . . . . 40

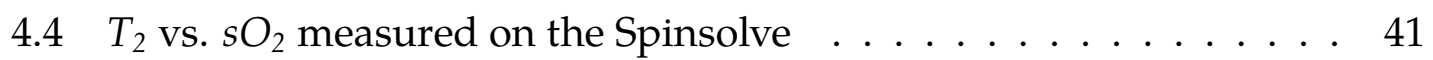

4.5 Stopped flow $\mathrm{T}_{2}$ vs. $\mathrm{sO}_{2}$ measured on the Halbach system . . . . . 42

4.6 Stopped flow $T_{2}$ vs. $\mathrm{sO}_{2}$ measured on the MOLE system . . . . . . 43

4.7 Change in $R_{2}$ for the three stopped flow systems . . . . . . . . . 44

4.8 Centrifuged blood samples from start and end of experiment . . . . 46

5.1 Experimental setup for continuous flow experiments (gas mixer and analyser not shown $) \ldots \ldots \ldots \ldots$. . . . . . . . . . . 51 
5.2 Examples of CPMG echo trains for doped water samples at different flow rates . . . . . . . . . . . . . . . . . . 5 53

5.3 Measured $T_{2}$ values at different flow rates . . . . . . . . . . . . 54

5.4 Spikes in continuous $T_{2}$ measurements . . . . . . . . . . . 55

5.5 PGSE data showing flow stability . . . . . . . . . . . . . . 56

5.6 Flow rate stability for different continuous flow setups . . . . . . 57

$5.7 \quad T_{2}$ measurements during blood oxygenation ramping at $40 \mathrm{MHz}$ field 58

$5.8 T_{2}$ measurements during blood oxygenation ramping at $20 \mathrm{MHz}$ field 60

$5.9 T_{2}$ measurements during blood oxygenation ramping at $14 \mathrm{MHz}$ field 62

$5.10 T_{2}$ measurements during blood oxygenation ramping at $10 \mathrm{MHz}$ field 64

$5.11 \mathrm{~T}_{2}$ measurements during blood oxygenation ramping at $5 \mathrm{MHz}$ field 66

$5.12 \mathrm{R}_{2}$ vs $\mathrm{sO}_{2}$ at different field strengths . . . . . . . . . . . . 68

5.13 Change in $R_{2}$ at different fields . . . . . . . . . . . . . . . . 69

5.14 Relationship between $\mathrm{K}_{0}$ on $\mathrm{sO}_{2}$ at different fields $\ldots \ldots$. . . . 70

$5.15 K_{0}$ slope dependence on $B_{0} \ldots \ldots \ldots \ldots \ldots \ldots$. . . . . . . . . . . . . . . .

5.16 Absorption spectra for samples of separated plasma in $5 \mathrm{MHz}$ experiment ....................... 76

5.17 Blood haemolysis experiment for $5 \mathrm{MHz}$ and $40 \mathrm{MHz}$ results . . . . 77

5.18 Back-predicted $\mathrm{s}_{2}$ from $\mathrm{NMR}$ measurements at $14 \mathrm{MHz}$. . . . . . 80

6.1 Different predictions of Luz-Meiboom and Jensen Chandra models 84

6.2 CPMG decays measured at the field of $20 \mathrm{MHz}$, at the different echo times. . . . . . . . . . . . . . . . . 86

6.3 Luz-Meiboom and diffusion model fits at different fields . . . . . . 89

6.4 Field dependence of $G_{0}$ and $K_{0} \quad \ldots \ldots \ldots$ 


\section{List of Tables}

3.1 Tuning and matching settings used in the continuous flow experi-

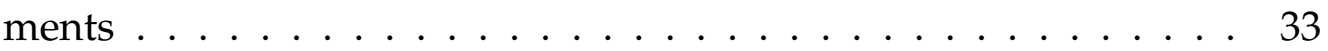

5.1 Measured $T_{2}$ dependence on speed . . . . . . . . . . . . 54

5.2 Fit parameters for dependence of $\mathrm{K}_{0}$ on $\mathrm{sO}_{2} \ldots \ldots \ldots \ldots$

6.1 Previous studies of $T_{2}$ shortening and dependence on exchange time 82

6.2 Best fit values to the experimental data at different fields . . . . . . 87 


\section{List of Acronyms}

BOLD Blood Oxygenation Level Dependent

CPB Cardiopulmonary Bypass

CPD Citrate/Phosphate/Dextrose

CPMG Carr Purcell Meiboom Gil

FID Free Induction Decay

fMRI functional MRI

Hct Haematocrit

MOLE Mobile Lateral Explorer

MRI Magnetic Resonance Imaging

NIRS Near-Infrared Spectrocsopy

NMR Nuclear Magnetic Resonance

PGSE Pulsed Gradient Spin Echo

RBC Red Blood Cell

RF Radio Frequency

SSR Sum of Squared Residuals 


\section{Chapter 1}

\section{Introduction}

Blood oxygenation is a critical parameter for assessing patient health and the management of disease. Oxygen saturation measurements are a routine part of care, and changes in these measurements can act as warning signs of acute/serious problems in the body. Low levels of oxygen can cause tissue damage and cell death, particularly in sensitive tissue such as the brain, where even brief periods of hypoxia can have catastrophic effects. Low oxygen levels can also be an issue for neonates, who can have circulatory and pulmonary systems which are still developing. Being able to quickly detect changes in oxygenation means that action can be taken to limit and/or prevent ill effects to patients.

The typical method for measuring blood oxygenation in patients is through pulse oximetry, a non-invasive method which measures the change in absorbance at various wavelengths of light to detect the presence of oxygenated and deoxygenated haemoglobin. This method can produce quick measurements of the blood oxygen saturation, while also being relatively cheap and robust. As it measures the blood, this technique provides systemic information about Oxygen saturation, measured at a single point on the finger or ear lobe. However, these blood saturation values are not necessarily representative of Oxygen levels in specific parts of the body. Problems with blood flow (ischemia) to sensitive areas in the body, can cause significant damage, and do not necessarily show up on pulse oximetry. Because of this, physicians desire local tissue oxygenation measurements, but they are difficult to measure.

Techniques for measuring local tissue oxygenation in the brain include the use of NIRS (Near-Infrared Spectroscopy), and the use of oxygen measuring polarographic electrodes. NIRS is a non-invasive method where the changes in light absorption from oxy / deoxy-haemoglobin are observed at near-infrared wavelengths, 
which can penetrate into tissue, similar to pulse oximetry. This technique is commercially available and already used regularly, particularly for monitoring neonates. However, it can be difficult to measure the local oxygenation of deeper structures, as it depends on light traveling through the tissue and returning to the sensor. Oxygen electrodes can also be used, which can be inserted into the desired area for measurement. These give a direct readout of the $\mathrm{pO}_{2}$, in the tissue surrounding the probe. However, inserting these oxygen electrodes into the tissue is extremely invasive, which has limited the use of these sensors.

Nuclear Magnetic Resonance (NMR) presents an alternative way to measure blood oxygen saturation. It has been known since the early 1980s that the saturation of blood is linked to its transverse relaxation time $T_{2}$. This relationship is caused by the change in the magnetic properties of haemoglobin when bound to oxygen. This effect is a contributing factor to BOLD (Blood Oxygen Level Dependent) contrast which underlies fMRI (functional Magnetic Resonance Imaging). Non-invasive measurement of local oxygen saturation has also been demonstrated using this effect. These applications have been developed using high-field ( $>1.5$ T) imaging systems, whose large size and cost means that these techniques are not in clinical use.

In the last 25 years, developments in portable magnetic resonance, using single sided magnets and coils, have meant that NMR can be used outside of laboratories and MRI suites. These magnetic resonance instruments produce a sweet spot, where the magnetic field and coil sensitivity combine to produce an NMR signal from protons inside this region. Combining this with the oxygen dependent $T_{2}$ change could produce an instrument which is sensitive to local changes in tissue oxygenation. However, single sided NMR systems have limitations on the strength and homogeneity of the magnetic field they produce, due to the use of permanent magnets, and the geometry of the system. This means that it is important to understand how factors like field strength and homogeneity affect how oxygenation dependent $T_{2}$ changes can be observed in this type of system.

In this thesis, this parameter space has been mapped out to determine how these factors affect the magnitude of the oxygenation $T_{2}$ effect in whole blood. Chapter 2 presents background information on NMR relaxation, the transport of oxygen in blood, and the origin of the oxygenation dependent $T_{2}$ change. Chapter 3 describes the two versions of the experimental setup used for the two series of experiments in this thesis. Chapter 4 presents the results of stopped flow experiments into the $T_{2}$ change at a series of oxygenation steps, while chapter 5 
presents the results of experiments measuring $T_{2}$ changes continuously as $\mathrm{sO}_{2}$ is slowly decreased. Chapter 6 evaluates an alternative model proposed by Jensen and Chandra to describe the process causing decreases in $T_{2}$. 


\section{Chapter 2}

\section{Background}

\subsection{Nuclear Magnetic Resonance}

Nuclear Magnetic Resonance (NMR) is an effect most often associated with chemical spectroscopy, or magnetic resonance imaging. This work in this thesis is closer to the technique of NMR relaxometry, the study of relaxation. This section will give a brief overview of the physics of NMR, using the semi-classical model and the Bloch equations. It describes NMR occurring to protons $\left({ }^{1} \mathrm{H}\right)$, which is the most common nucleus used for NMR and what is used in this thesis, although NMR can be observed with any nuclei with non-zero spin. For a more complete discussion, the reader is directed to one of the many textbooks on the subject, including books by Abragham [1] and Callaghan [2] or Brown et al. [3].

Protons have a magnetic moment due to their intrinsic angular momentum, or 'spin'. To observe nuclear magnetic resonance, protons are put into a magnetic field $\left(B_{0}\right)$ that interacts with the protons magnetic moment, lowering the energy of magnetic moments aligned with $B_{0}$ (along $z$ ). Following the Boltzman distribution, this energy difference causes an increase in the fraction of protons in the lower energy state, generating a net magnetisation aligned with the $B_{0}$ field. As the size of the net magnetisation depends on the relative difference of spins towards and against the field, the net magnetisation becomes stronger when a larger $B_{0}$ field is used. Any imbalance created is typically very small, which means that the NMR signal generated is very weak.

A magnetic moment in a magnetic field experiences a torque, which causes it to rotate around an axis along the magnetic field. This is called Larmor precession, and gives rise to the Larmor frequency $\omega$, which defines the rate of rotation. Protons in a magnetic field precess at a frequency given by Equation 2.1, where $\gamma$ 

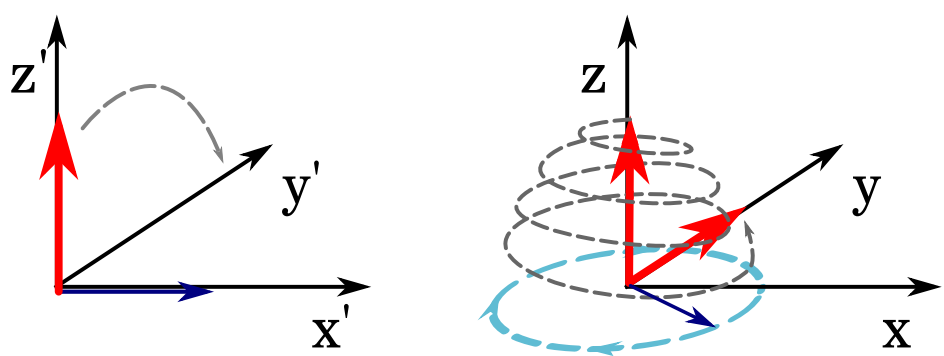

Figure 2.1: Applying a $B_{1}$ field along $x^{\prime}$ causes the magnetisation to rotate towards $y^{\prime}$. Transforming this to the laboratory frame shows that the $B_{1}$ field must oscillate at the precession rate, and causes the magnetisation to spiral down into the transverse $(x y)$ plane

is the gyromagnetic ratio $\left(2.56 \times 10^{8} \mathrm{rads}^{-1} \mathrm{~T}^{-1}\right.$ for protons $)$. At equilibrium, the net magnetisation is along $z$, so precession is not visible.

$$
\omega=\gamma B_{0}
$$

The effect of the $B_{1}$ field can be seen by considering a frame $\left(x^{\prime}, y^{\prime}, z^{\prime}\right)$ which rotates around the $z$ axis at this precession frequency (a 'rotating frame', when compared to the stationary 'lab frame') In the rotating frame, the net magnetisation still lies along the $z$-axis. Applying an additional magnetic field, along $x^{\prime}$ (i.e. perpendicular to $B_{0}$ ) to the $z$-axis causes the magnetisation to rotate around $x^{\prime}$ towards $y^{\prime}$, following the same precession principle. The strength and time that the $B_{1}$ field is applied for can be changed to control the amount of rotation, leading to $90^{\circ}$ and $180^{\circ}$ pulses.

Transforming this into the lab frame means that the $B_{1}$ field becomes a magnetic field oscillating at the Larmor frequency, while the components of the magnetisation along $x$ and $y$ correspond to a rotating magnetisation in the sample. This produces the resonance condition for NMR - the frequency of the $B_{1}$ field needs to match the precession frequency to cause maximum rotation. These can be created and detected using a coil, as the rotating magnetic field from the sample induces a voltage in the coil due to Faraday's Law. This NMR signal is known as the Free Induction Decay (FID). 


\section{$2.2 \quad T_{1}$ and $T_{2}$ relaxation}

The NMR signal from a sample decays due to relaxation with two main mechanisms. It can be caused by the return of net magnetisation to equilibrium, or by a loss of coherence across a sample.

As mentioned above, the equilibrium state of a sample in a $B_{0}$ field is for the net magnetisation to align with the $B_{0}$ magnetic field. When this is disturbed, for example, in an NMR experiment or when the sample is first placed in the $B_{0}$ field, the protons in the sample exchange energy to return to the equilibrium. This process, also known as spin-lattice relaxation, is described by Equation 2.2, where $\mathrm{M}_{0}$ is the equilibrium magnetisation, and the time constant for relaxation is $T_{1}$.

$$
\frac{\mathrm{d} M_{z}}{\mathrm{~d} t}=\frac{1}{T_{1}}\left(M_{0}-M_{z}\right)
$$

The $T_{1}$ of a sample can be determined by an inversion recovery experiment. A $180^{\circ}$ pulse is applied to the sample, and the magnetisation remaining is measured after a range of variable delay time $\left(t_{d}\right)$ by applying a $90^{\circ}$ pulse and taking the intensity of the FID. This can be used to fit a curve with the equation

$$
M\left(t_{d}\right)=M_{0}\left(1-2 e^{-\frac{t_{d}}{T_{1}}}\right)
$$

In most cases, the $T_{1}$ controls how rapidly an experiment can be repeated. A general rule is to set the repetition time $\left(T_{R^{-}}\right.$the delay between experiments) to $5 \times T_{1}$, so that the sample magnetisation recovers to $>99 \%$ of its original equilibrium level. Otherwise the signal produced will be weaker, as it depends on the magnetisation along the $z$ axis. This also removes any remaining transverse magnetisation, as the protons return to equilibrium. Because of this, $T_{2}<T_{1}$.

The $x$ and $y$ components of a sample's magnetisation (the transverse magnetisation) also undergoes relaxation. The transverse magnetisation is generated by the combination of magnetisation from protons across the sample. The NMR signal is generated when the magnetic moments of all of the protons in a sample precess at the same rate, and are all aligned in the same direction (i.e. have the same phase), creating coherence. If protons in part of the sample experience a different magnetic field, they precess at a different rate and lose coherence with the rest of the sample. This reduces the overall transverse magnetisation of the sample, which decreases following Equation 2.3, where $T_{2}$ is the time constant for this relaxation.

$$
\frac{\mathrm{d} M_{x y}}{\mathrm{~d} t}=-\frac{1}{T_{2}}\left(M_{x y}\right)
$$




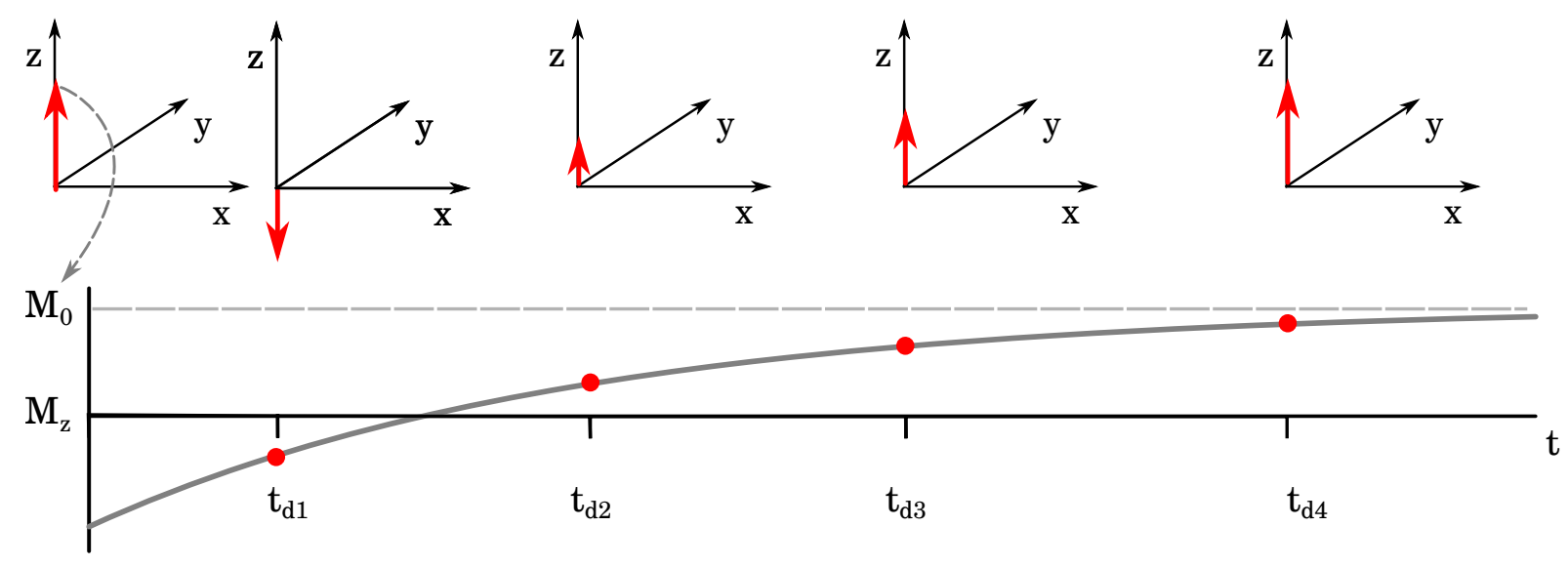

Figure 2.2: $T_{1}$ relaxation leads to the recovery in the equilibrium magnetisation $\left(\mathrm{M}_{0}\right)$. The Inversion Recovery experiment works by applying a $180^{\circ}$ pulse, then measuring the magnetisation by applying a $90^{\circ}$ pulse at time $t_{d}$ to generate an FID. Repeating the experiment with a range of $t_{d}$ s allows the samples $T_{1}$ to be measured.

In a physical sample, protons are always experiencing different magnetic fields due to the fluctuating magnetic moments of surrounding protons. The dephasing from this process is random and non-reversible. The physical properties of a sample control the amount of interaction between protons on surrounding molecules, and the rates of this intrinsic $T_{2}$ relaxation, for example, solids have extremely fast $T_{2}$ relaxation times, on the order of 10 s of microseconds, while water can have a $T_{2}$ on the order of seconds.

$T_{2}$ relaxation can also be increased by the presence of paramagnetic ions in the sample, such as $\mathrm{Cu}^{2+}, \mathrm{Mn}^{2+}$ and $\mathrm{Gd}^{3+}$. These affect the magnetic fields experienced by protons in the sample and cause additional dephasing. Their effect is characterised by a relaxivity $r$, measured in $\mathrm{s}^{-1} / \mathrm{mmolL}^{-1}$, which describes the dependence on concentration. This is due to the additive nature of relaxation rates: when multiple processes cause relaxation, the relaxation rates add together

$$
\frac{1}{T_{2}}=R_{2}=\frac{1}{T_{2 \text { intrinsic }}}+r_{2} c_{\text {metal }}
$$

Additionally, another process reduces the transverse magnetisation of a sample. $T_{2}{ }^{*}$ relaxation is associated with the homogeneity of the $B_{0}$ field, as different magnetic fields across the sample will produce different precession rates, and cause protons to dephase. This process is illustrated in Figure 2.3. 

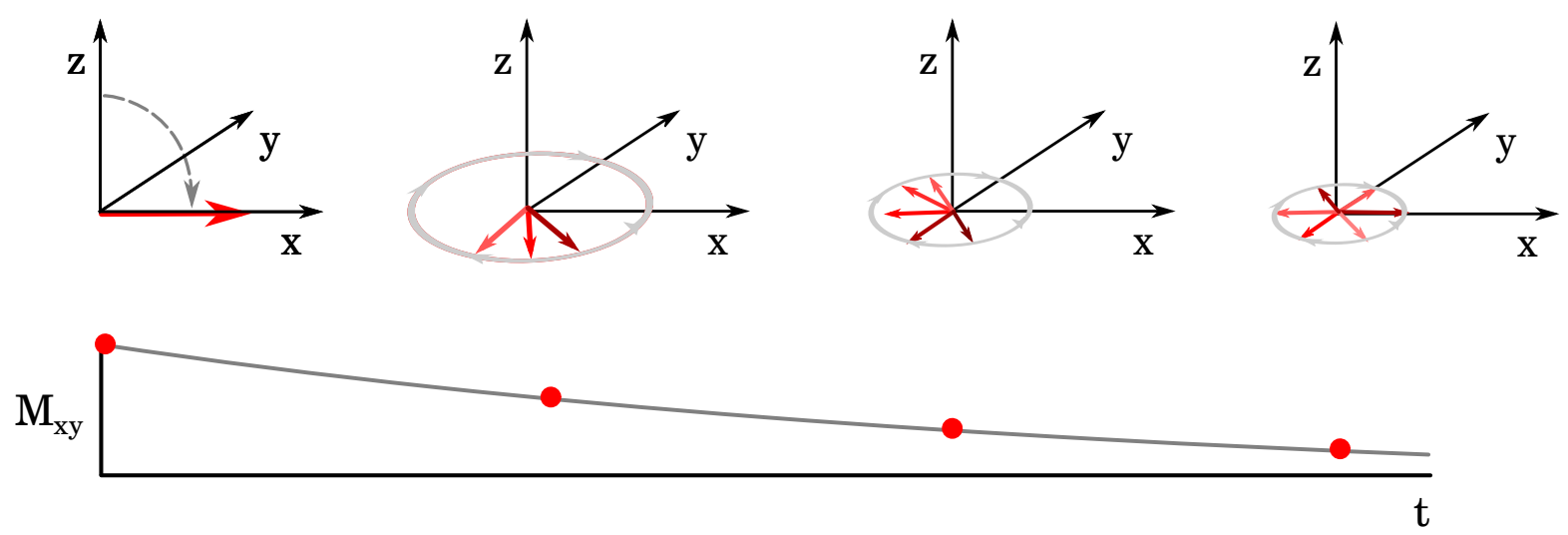

Figure 2.3: Variations in the $B_{0}$ field cause protons in different locations to precess at different rates (shown in red shading). As the total magnetisation is the sum of the proton magnetic moments, the different magnetisations start cancelling out and cause a decay in the transverse component of the magnetisation, called $T_{2}{ }^{*}$ relaxation.

The precession of the net magnetisation around the $B_{0}$ field, and the effect of relaxation on the net magnetisation can be expressed using the phenomelogical Bloch equation Equation 2.4.

$$
\frac{\mathrm{d} \mathbf{M}}{\mathrm{d} t}=\gamma \mathbf{M} \times \mathbf{B}+\frac{1}{T_{1}}\left(M_{0}-M_{z}\right)-\frac{1}{T_{2}} M_{x y}
$$

\subsection{Spin echoes and CPMG}

The spin echo was first described by Hahn in 1950[4]. By applying a $180^{\circ}$ pulse a time $\tau$ after a $90^{\circ}$ pulse, he observed a signal forming at $2 \tau$. He showed that this is due to the spread of phase caused by the $B_{0}$ field inhomogeneity being reversed, which allows the protons to rephase and regenerate a net transverse magnetisation that can be detected.

The formation of the spin echo is shown illustrated in Figure 2.4. The inhomogeneous $B_{0}$ field causes protons across the sample to have different Larmor frequencies, meaning that they precess at different rates, and accumulate different amounts of phase as time continues. The different phases cause the net transverse magnetisation of the whole sample to decay, with a shorter time constant $T_{2}{ }^{*}$. Applying a $180^{\circ}$ refocusing pulse $\tau$ after the $90^{\circ}$ pulse causes the magnetic moments of each proton to rotate through $180^{\circ}$ around the $x$ (or $y$ ) axis, so that the differ- 

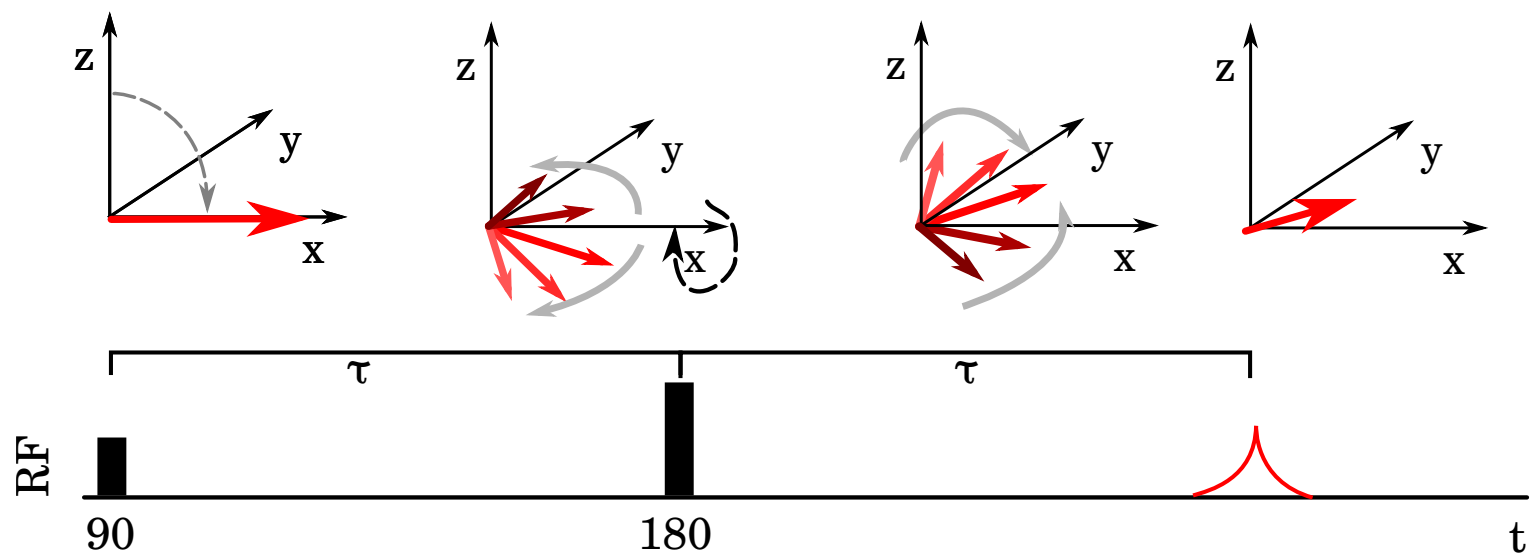

Figure 2.4: Generation of a spin echo: as protons precess at different rates, they accumulate different amounts of phase (shown by red shading). Applying a $180^{\circ}$ pulse a time $\tau$ after the $90^{\circ}$ pulse causes the magnetisation to be rotated around the $x$-axis, so the different rates unwind their different phases, producing an echo at $2 \tau$.

ent precession rates unwind the different accumulated phase. Thus unwinding causes the magnitude of the net transverse magnetisation to increase, reaching a maximum at $2 \tau$, which is the centre of the echo. The spin echo will still have a reduced intensity due to the intrinsic $T_{2}$ relaxation processes described above. However, it removes the effects of the inhomogeneous $B_{0}$ field, and allows the measurement of the intrinsic $T_{2}$ relaxation process.

This rephasing process relies on the protons experiencing the same $B_{0}$ field in the period before and after the $180^{\circ}$ pulse. If protons move across an inhomogeneous field during this time, they may not experience the same field and not be complete refocussed at $2 \tau$. This can be due to diffusion (the random movement of protons in the sample) or processes such as chemical exchange (explored below).

Once the magnetisation has been refocused in the spin echo, the transverse magnetisation decays following the $T_{2}{ }^{*}$ relaxation time again. Carr and Purcell proposed repeating the refocussing pulses, to create more echoes and further map out the $T_{2}$ relaxation [5]. In this experiment, the refocussing pulses are evenly spaced by an echo time, as shown in Figure 2.5. Meiboom and Gill improved this experiment by adjusting the phase of the $180^{\circ}$ pulses relative to the $90^{\circ}$ pulse, such that they rotate the magnetisation around the $y$-axis rather than $x$-axis [6]. This reduces the errors caused by an incorrect $180^{\circ}$ pulse. With this modification, the CPMG experiment is commonly used for fast and robust measurements of the $T_{2}$ 


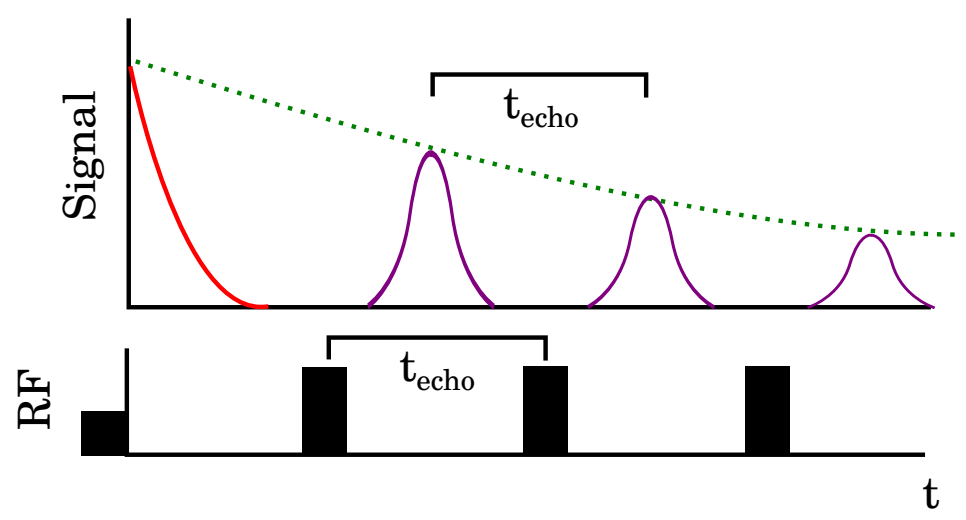

Figure 2.5: Repeatedly applying $180^{\circ}$ pulses produces a series of spin echoes. Measuring the intensity of the echoes (purple) allows the true $T_{2}$ relaxation (green) to be measured.

of a sample.

$$
S(t)=S_{0} e^{\frac{t}{T_{2}}}
$$

The intensity of echoes in a CPMG experiment follows a monoexponential decay (for samples with a single relaxation component). The $T_{2}$ can be measured by the slope of a semi-log plot, or by non-linear fitting of the function 2.5. While measurements of the intrinsic $T_{2}$ should not be affected by the echo time used, process like exchange and diffusion can alter the measured $T_{2}$. For example, for protons moving in a magnetic gradient, a longer echo time means that protons experience a wider range of $B_{0}$ fields before being refocussed. This makes the refocusing less effective, and results in a shorter observed $T_{2}$, following Equation 2.6. Similarly, exchange processes can cause protons to experience different magnetic fields in the timescale of the experiment, causing the refocusing to be less efficient. Exchange processes, and their applicability to blood are discussed further in section 2.8 .

$$
\frac{1}{T_{2}}=\frac{1}{T_{2}}+\frac{\gamma^{2} G^{2} D \tau^{2}}{12}
$$

\subsection{PGSE experiments}

Pulsed Gradient Spin Echo (PGSE) experiments combine the spin echo with magnetic field gradients to measure the displacement of protons in a sample. 

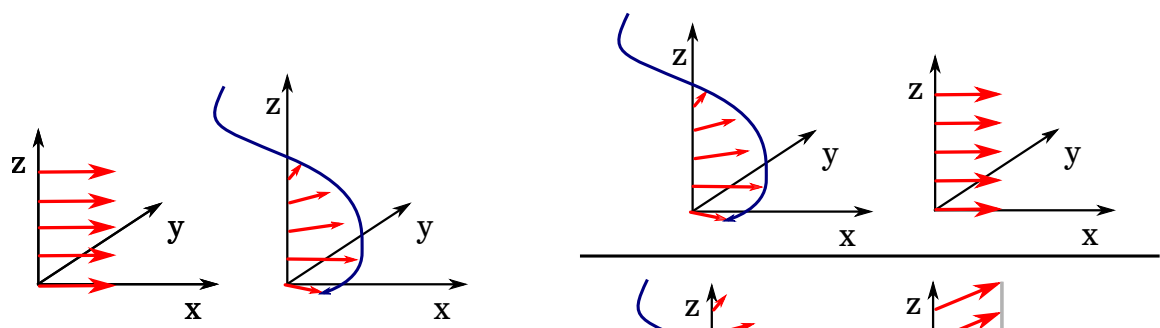

$\Delta$
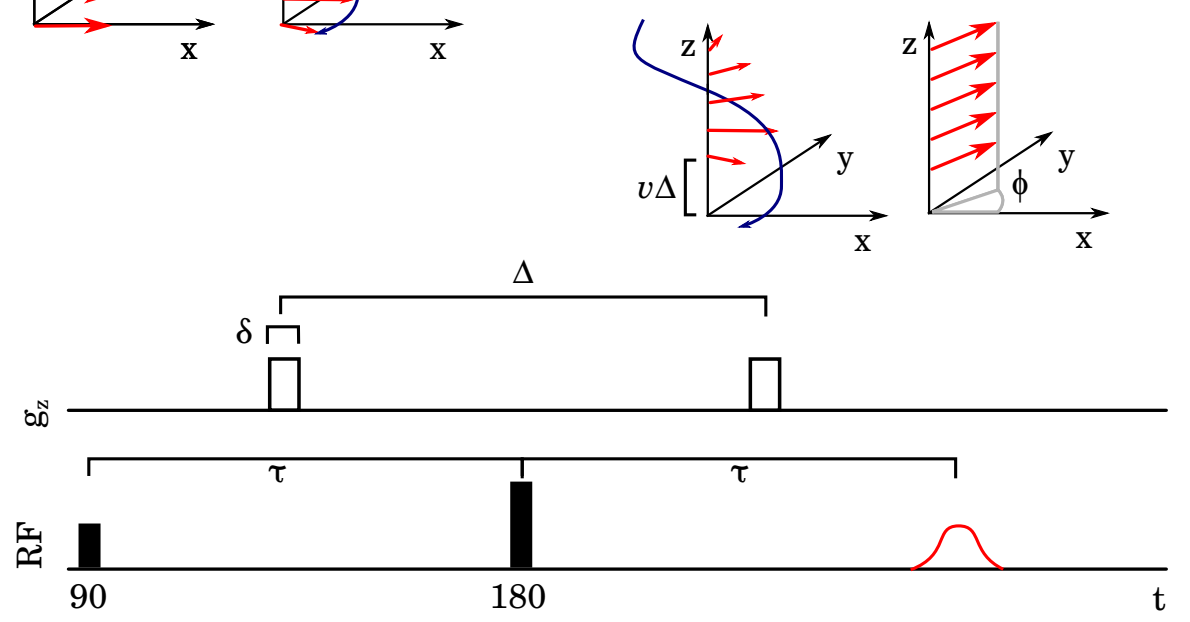

Figure 2.6: Pulsed Gradient Spin Echo experiment: Applying a gradient changes the precession frequency to be dependent on the position along $\mathrm{z}$, and imparts a phase difference depending on the time it is active ( $\delta$ ) (shown by the blue helix). Using a spin echo, and repeating the gradient pulse will unwind the this added phase if the protons have not moved (upper image). If the protons have moved together (e.g. flow), the second gradient pulse will bring them back into phase with each other, but leave an additional phase $(\phi)$ which can be measured to determine the displacement.

Traditionally, a magnetic field gradient creates a known change in the $B_{0}$ field, which is linearly related to its position: $B_{0}(z)=B_{0}+g(z)$ This allows protons to be spatially resolved, as they will have different Larmor precession frequencies at different locations. This principle is the basis of Magnetic Resonance Imaging (MRI).

Stejskal and Tanner proposed the PGSE experiment in 1965 [7]. Their experiment uses a pulsed magnetic field gradient, which creates a range of precession rates across the sample for a short time, so that protons accumulate a phase related to their position. A $180^{\circ}$ pulse is then applied, and an identical pulsed field gradient is reapplied. This causes the phase accumulated in the first pulse to be reversed before the spin echo is formed. Assuming there is no movement, the protons in the sample will be completely rephased, and the normal spin echo will be recovered. 
Diffusion (random movement) of protons means that the rephasing is not as effective, as the phase accumulated in the first pulsed gradient will not be totally removed. This means that some of the protons will cancel each other out, and cause the intensity of the spin echo to be reduced. By running experiments with a range of gradient strengths $(g)$ and measuring the echo intensity, the diffusion coefficient (D) for the sample can be obtained using Equation 2.7, where $\delta$ is the length of time the pulsed gradient field is active, and $\Delta$ is the time between the two gradient pulses.

$$
S(G)=S_{0} e^{-\gamma^{2} g^{2} \delta^{2}\left(\Delta-\frac{\delta}{3}\right) D}
$$

In contrast, uniform motion of protons in a sample (such as flow) produces a different effect. Rather than the intensity of the spin echo decreasing, the phase of the spin echo is shifted. That is, the spin echo will form with the transverse magnetisation rotated slightly around the $x y$ plane when compared to a measurement with no flow (or no gradient), as the second gradient pulse 'overcorrects' the dephasing. The velocity of the sample can be determined using two methods $[8,9,10]$. By measuring the phase and intensity at a range of gradient strengths, the velocity can be found using a Fourier transform. This produces a spectrum with a peak at the propagator, the average displacement in the sample. Alternatively, taking the phase $(\phi)$ of the echo measured with a single gradient strength, and comparing it to a reference where there is no gradient, the flow velocity can be measured using Equation 2.8. This second 'one-shot' method was used in these experiments, as it requires less measurements than the Fourier method to complete the same measurement.

$$
v=\frac{\phi}{\gamma \Delta \delta g}
$$

\subsection{Blood and oxygen saturation}

Blood contains multiple components, including red blood cells, white blood cells, platelets, and plasma. Red Blood Cells (RBCs) are associated with oxygen transport, while white blood cells are part of the immune system. Platelets are involved in the blood clotting process, and plasma is the mixture of water, nutrients and proteins which these components are suspended in. Blood is pumped around the circulatory system by the heart, and transports nutrients to all parts of the body. On average, blood makes up 7\% of a person's body weight, making a volume of about $5 \mathrm{~L}$. 


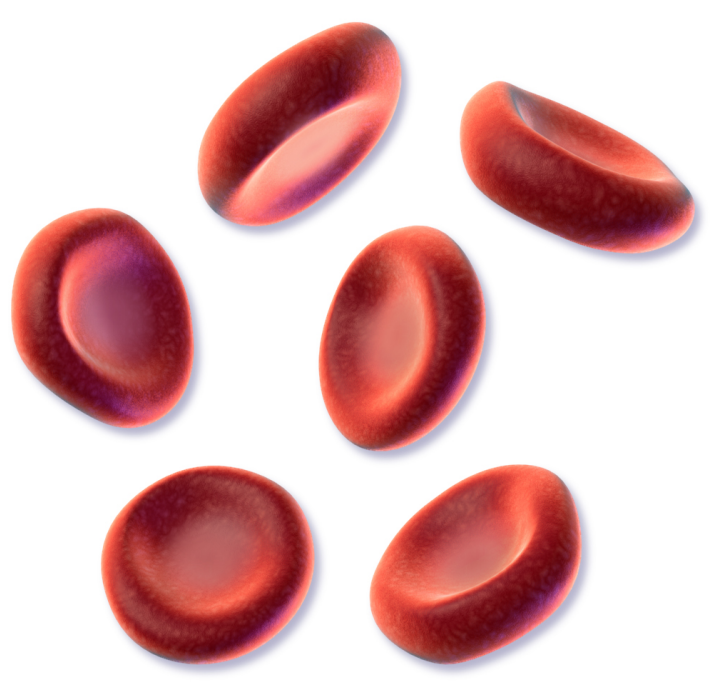

Figure 2.7: Illustration of red blood cells. Source: Bruce Blaus https : //commons . wikimedia.org/wiki/File:Blausen_0761_RedBloodCells.png

In addition to the nutrients carried in the plasma, blood is responsible for the transport of oxygen from the lungs to the body. Most of the oxygen transported is carried by red blood cells, with a small amount dissolved in blood plasma (typically $<3 \%$ ). These are shown in Figure 2.7, and have a biconcave disc shape, with a mean diameter of $7.8 \mu \mathrm{m}$, and mean thickness of $2.5 \mu \mathrm{m}$. RBCs contain no cell nucleus and are very deformable, which allows them to travel through narrow capillaries in tissue. While they have no nucleus, they still metabolise slowly to maintain membrane deformability and ion transport through the cell membrane. RBCs have a typical lifetime in the body of 120 days.

The fraction of blood volume in red blood cells is called the haematocrit (typically around 0.4 in healthy adults) The primary role of RBCs is to contain and transport haemoglobin in the blood, and they can contain very high concentrations (up to $34 \mathrm{~g} / \mathrm{L}$ ) of haemoglobin inside.

\subsection{Oxy- and deoxy-haemoglobin}

Haemoglobin is a protein which can reversibly bind to oxygen to improve its transport in the body [11]. By binding the oxygen to haemoglobin, blood can transport 30-100 times more oxygen than relying on dissolved oxygen in the plasma. It contains four heme groups, each containing an iron ion $\left(\mathrm{Fe}^{2+}\right)$ which can reversibly bind to oxygen. In the lungs, oxygen diffuses into the blood and RBCs, and is taken 
up by haemoglobin to form oxy-haemoglobin. This increases the oxygen saturation $\left(\mathrm{sO}_{2}\right)$, which is defined as the fraction of all haemoglobin bound to oxygen in Equation 2.9 (in terms of concentration) [12].

$$
s \mathrm{O}_{2}=\frac{c_{o x y H b}}{c_{\text {oxyHb }}+c_{\text {deoxyHb }}}
$$

After leaving the lungs, the $\mathrm{sO}_{2}$ will be around $97 \%$, and the $\mathrm{pO}_{2}$ (the partial pressure of oxygen - proportional to the concentration of $\mathrm{O}_{2}$ ) will be around $95 \mathrm{mmHg}$. As blood travels around the body, oxygen diffuses from the blood into the tissue, which has a lower $\mathrm{pO}_{2}$ due to metabolism. The oxygen dissolved in plasma decreases, decreasing the $\mathrm{pO}_{2}$. This change triggers the oxy-haemoglobin to release oxygen, forming deoxy-haemoglobin and effectively buffering the $\mathrm{pO}_{2}$ change. In venous blood, after leaving tissue, the $\mathrm{sO}_{2}$ will be between $20 \%$ to $70 \%$, and have a $\mathrm{pO}_{2}$ between $20 \mathrm{mmHg}$ to $40 \mathrm{mmHg}$ (depending on the demand for oxygen in the tissue) [11].

In addition to these two forms of haemoglobin, other dys-haemoglobins can also be created, including met- and carboxy-. Met-haemoglobin is formed when the iron in the complex is further oxidised to $\mathrm{Fe}^{3+}$. Carboxy-haemoglobin is formed when carbon monoxide ( $\mathrm{CO}$ ) binds to haemoglobin, forming a bond much stronger than oxygen does, leading to the loss of oxygen transport capacity. These other forms are not expected to be created in this experiment.

The relationship between $\mathrm{sO}_{2}$ and $\mathrm{pO}_{2}$ is non-linear due to effects such as cooperative binding. Binding the first oxygen molecule to one of the heme groups causes a change in the shape of the protein which makes binding at the other heme groups easier. This makes the $\mathrm{sO}_{2}$ increase more steeply (as a function of $\mathrm{pO}_{2}$ ) once the first oxygen is bound. This effect also works in reverse, causing the protein to release oxygen. Physiologically, this causes a release of oxygen in areas with low $\mathrm{pO}_{2}$, which helps to deliver oxygen to tissue that needs it.

Other factors also affect the relationship between $\mathrm{sO}_{2}$ and $\mathrm{pO}_{2}$ [11]. One example is the $\mathrm{pH}$, where an increase in acidity (e.g. 7.4 to 7.2 ) causes a decrease in oxygen affinity, and the release of more oxygen from haemoglobin. This dependency underlies the Bohr effect: $\mathrm{pH}$ in the blood is also affected by dissolved $\mathrm{CO}_{2}$, which exists in equilibrium with carbonic acid in the blood. An increased $\mathrm{pCO}_{2}$ causes more carbonic acid to be formed, lowering the $\mathrm{pH}$ of the blood. This causes oxygen to be released in areas where $\mathrm{CO}_{2}$ is being produced due to metabolism.

Changes in the $\mathrm{sO}_{2}$ can be measured using the different optical and magnetic properties of oxy- and deoxy-haemoglobin. The binding of oxygen to haemoglobin 


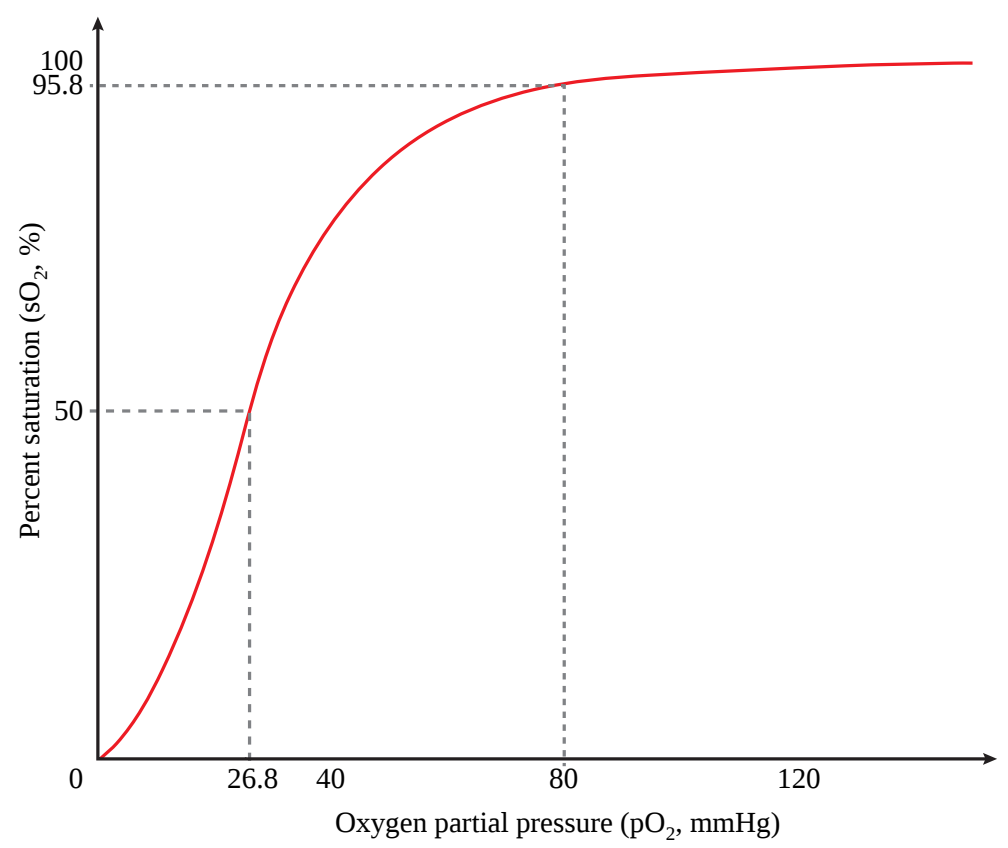

Figure 2.8: Graph showing relationship between $\mathrm{pO}_{2}$ and $\mathrm{sO}_{2}$ in human blood. Source: https://commons.wikimedia.org/wiki/File:Hemoglobin_saturation_ curve.svg

causes them to have slightly different optical absorption spectra. In particular, the deoxy-haemoglobin absorbs strongly in the red region of the spectrum. This causes a colour change, where oxygenated blood appears red, and deoxygenated blood appears almost black.

Oxygen binding to haemoglobin also causes a change in the electronic structure of the iron atom [13]. In the oxygenated state, the iron atom complex has no unpaired electrons. In the deoxygenated state however, the iron complex contains unpaired electrons, making it paramagnetic. This increases the magnetic susceptibility of the cytoplasm inside the RBC. Changes in susceptibility across a sample produce inhomogeneity in the $B_{0}$ magnetic field, and the field generated by the susceptibility difference between RBC and plasma creates the decreasing $T_{2}$ effects used in this thesis. The mechanism for this decrease is described in section 2.8 below.

\section{$2.7 \mathrm{sO}_{2}$ measurement}

The gold standard method for measuring the $\mathrm{sO}_{2}$ of blood is co-oximetry, which relies on the optical absorption changes in oxy-/deoxy- haemoglobin [12]. This 
method typically uses spectroscopic measurements of absorption at multiple wavelengths to identify the concentrations of oxy-haemoglobin, deoxy-haemoglobin and other dys-haemoglobins in a blood sample.

Alternatively, the $\mathrm{sO}_{2}$ can be found using knowledge of the $\mathrm{pO}_{2}$ of the sample, and the relationship between $\mathrm{pO}_{2}$ and $\mathrm{sO}_{2}$. The $\mathrm{pO}_{2}$ of a sample can be measured using a Clark electrode, which uses a redox reaction with a rate dependent on the $\mathrm{pO}_{2}$. The Clark electrode contains a platinum/silver cathode/anode pair behind an oxygen permeable membrane. Oxygen is reduced to water following the chemical equation below, and the current generated by the electrode is proportional to $\mathrm{pO}_{2}$. This method is used in the iStat in this thesis to measure $\mathrm{sO}_{2}$.

$$
\mathrm{O}_{2}+4 \mathrm{e}^{-}+4 \mathrm{H}^{+} \rightarrow 2 \mathrm{H}_{2} \mathrm{O}
$$

These methods both require taking samples of blood to measure. Pulse oximetry is a non-invasive technique which is commonly used to measure $\mathrm{sO}_{2}$ in patients. A pulse oximeter shines light through a part of the patient, typically a finger, and measures the light transmitted or reflected from the tissue. The absorbance from the tissue can be rejected by using the fact that the arterial blood flow is pulsatile, meaning that the component of absorption varying in time can be assigned to the blood [14]. The differing absorbance at multiple wavelengths is used to find the fraction of oxy-haemoglobin in blood.

While in theory, this could be done using the Beer-Lambert law with known extinction coefficients of oxy- and deoxy-haemoglobin, effects such as scattering from red blood cells mean that empirical calibration methods are used to convert the measured light intensities to $\mathrm{sO}_{2}$ [14]. In two wavelength pulse oximeters, like used in this thesis, a quadratic calibration curve is used to approximate the relationship between the ratio of light intensities $\mathrm{R}$, and the $\mathrm{sO}_{2}$. Pulse oximeters with more than two wavelengths can apply more advanced algorithms to measure $\mathrm{sO}_{2}$. These devices are very common in clinical practice, and are relatively cheap and robust.

\section{8 $\quad T_{2}$ changes due to oxygenation in blood}

As mentioned above, changes in oxygenation cause the fraction of haemoglobin bound to oxygen to change. Decreasing the oxygenation means there is more deoxy-haemoglobin which, due to its increased paramagnetism, causes a larger susceptibility change between the RBC cytoplasm and the surrounding plasma. 
While the change between intracellular and extracellular is difficult to measure, this change can be measured for whole samples of blood, and found to be $\Delta \chi_{D O}=$ $0.27 \mathrm{ppm}$ (cgs) [15]. Changes in susceptibility cause variations in the magnetic field, which causes the refocusing pulses in the CPMG experiment to be less effective in recovering the phase. This causes increased dephasing, which is observed as a decrease in $T_{2}$.

Thulborn introduced the use of the Luz-Meiboom equation to describe the effect of $\mathrm{sO}_{2}$ on the $\mathrm{T}_{2}$ of blood. This equation comes from the study of a chemical exchange process, where protons on an ammonium ion exchange with the solvent [16]. Protons bound to the ammonium ion have a different chemical shift, which combined with the exchange, causes increased dephasing and a shorter $T_{2}$. Luz and Meiboom show that this process leads to a $T_{2}$ decrease dependent on the echo time given by Equation 2.11 [16], where $p_{i}$ is the fraction of protons in state $i, \delta_{i}$ is the shift of protons in state $i, t_{e c}$ is the echo time, and $\tau_{e x}$ is the average time between exchanges. It also includes $T_{20}$ to account for the $T_{2}$ when there is no exchange contribution.

$$
\frac{1}{T_{2}}=\frac{1}{T_{20}}+\sum_{i} p_{i} \delta_{i}^{2} \tau_{e x}\left(1-\frac{2 \tau_{e x}}{t_{e c}} \tanh \frac{t_{e c}}{2 \tau_{e x}}\right)
$$

Some authors have proposed that this is a similar situation to red blood cells, where the susceptibility change due to haemoglobin causes a different $B_{0}$ field in the cytoplasm when compared to the plasma, and protons exchange across the cell membrane [17]. Others have proposed that the dephasing is caused by diffusion through intracellular and/or extracellular gradients generated by the susceptibility change $[18,19,20]$. The exchange time then becomes the time for spins to experience the range of fields in the gradient.

Wright [21] applied this exchange model to blood, expressing it with more relevant parameters. In Equation 2.12 the summation over the states becomes the haematocrit, as this relative population of protons in cytoplasm and plasma, and the frequency difference given by a term dependent on the $\mathrm{sO}_{2}$, the field strength $\omega_{0}$, and a dimensionless factor $\alpha$ which is dependent on the susceptibility change of deoxy-haemoglobin, and the geometry of the red blood cell.

$$
\frac{1}{T_{2}}=\frac{1}{T_{20}}+\left(P_{A}\right)\left(1-P_{A}\right)\left[\left(1-s O_{2}\right) \alpha \omega_{0}\right]^{2} \tau_{e x}\left(1-\frac{2 \tau_{e x}}{t_{e c}} \tanh \frac{t_{e c}}{2 \tau_{e x}}\right)
$$

Jensen and Chandra investigated this problem using the weak-field approximation to derive expressions for the signal in a CPMG experiment from protons 
diffusing in a weakly inhomogeneous field [22]. This method uses a correlation function $K(t)$ that describes the variations in the magnetic field as protons diffuse. The true correlation function is normally not known analytically however, so approximations are used. By approximating this correlation function with a simple exponential decay Equation 2.13, they found that the relaxation rate in a CPMG experiment is given by Equation 2.14 [22].

$$
\begin{gathered}
K(t)=K_{0} e^{-t / \tau} \\
\frac{1}{T_{2}}=\frac{1}{T_{20}}+\gamma^{2} K_{0} \tau_{e x}\left(1-\frac{2 \tau_{e x}}{t_{e c}} \tanh \frac{t_{e c}}{2 \tau_{e x}}\right)
\end{gathered}
$$

Comparing Equation 2.14 with Equation 2.12 shows that it has a similar form to the Luz-Meiboom equation, but with a more general "correlation time" $\tau$, and the factor $K_{0}$ representing the variance of the magnetic field. Because of this, the correlation time is not directly connected to an exchange process, which could explain why the Luz-Meiboom formula agrees with experimental results, but also gives a range of exchange times. In practice, this $K_{0}$ parameter describes how dependent the $T_{2}$ is on echo time. A larger value of $K_{0}$ means that the inhomogeneities are stronger, and cause a larger dephasing effect as the echo time increases.

This exchange model is used in this research, as it is most commonly used in the literature. An alternative model which more accurately characterises the diffusion of protons around red blood cells has also been developed [22], and is investigated and compared with the exchange equation in chapter 6 .

\subsection{Literature on NMR measurement of oxygen satu- ration}

The effect of $\mathrm{s}_{2}$ on the $\mathrm{T}_{2}$ of blood was originally described by Thulborn in 1982, who investigated how $T_{2}$ changed as a function of oxygenation, magnetic field strength and haematocrit [23]. Thulborn found that:

$$
\frac{1}{T_{2}}=R_{2} \propto\left(100-s O_{2}\right)^{2}
$$

Using a variety of spectrometers and NMR systems, he also showed that the strength of this effect scales with $B_{0}$ squared, and that this effect requires that the red blood cells are intact (as no change in $T_{2}$ occurred when the cells were lysed.) 
From this initial study, other researchers studied how the effect can be detected at lower fields. Gomori measured the $T_{2}$ of samples of oxygenated and deoxygenated blood at fields ranging from $0.19 \mathrm{~T}$ to $1.4 \mathrm{~T}$, and found that changes were still visible at these lower fields [18]. This sort of experiment was also completed by Brooks, who found that $T_{2}$ decreases were visible at fields between $0.05 \mathrm{~T}$ and 1.5 T [19]. Brooks mapped out the dependence on field strength more finely than Gomori, and was able to show that the size of the effect still scales with $B_{0}$ squared at low fields. In these studies, the samples of blood were measured with either completely oxygenated and completely deoxygenated blood, typically obtained by adding sodium dithionite to deoxygenate the blood chemically. There are a handful of other studies of this effect at these low field strengths, such as Bryant [17] and Stadelmann [24], although like the studies by Brooks and Gomori, these were only done using samples of deoxygenated and oxygenated blood.

More recently, this effect has been studied in-vitro for applications in high field imaging systems. Silvennoin [25], Stefanovic [26], Chen [27] and Gardener [28] scanned samples of blood with varying oxygenation levels in MRI scanners (1.5, $2.35,3$ or $4.7 \mathrm{~T}$ ) to study the change in $T_{2}$ as a function of oxygenation, haematocrit and CPMG echo time. These studies showed that the effect follows the same quadratic trend found by Thulborn, and shows good agreement with the theoretical models discussed in chapter 6.

Another method of studying $\mathrm{T}_{2}$ changes with $\mathrm{sO}_{2}$ in-vitro was described by Meyer et al., who used a continuous flow loop to measure $T_{2}$ at varying levels of $\mathrm{sO}_{2}$, with a spectrometer at $4.7 \mathrm{~T}$ [29]. This method was more recently used by the group of Peter van Zijl, who has published multiple studies [30,31,32] on the dependence of $\mathrm{T}_{2}$ on $\mathrm{sO}_{2}$, haematocrit, and on field strength for imaging systems. These studies have looked at magnetic field strengths from 3.0 T to $16.4 \mathrm{~T}$.

The effect of $\mathrm{sO}_{2}$ on $\mathrm{T}_{2}$ has also been applied in in-vivo imaging, with a pioneering study by Wright [21], who measured changing oxygen saturation in the body using $T_{2}$ changes. More recently, other researchers have developed methods for imaging $\mathrm{sO}_{2}$ in blood based on this $T_{2}$ effect [33]. $T_{2}$ Relaxation Under Spin Tagging (TRUST)-MRI can be used to isolate the protons in blood, and find $s \mathrm{O}_{2}$ from $\mathrm{T}_{2}$ and a calibration curve [34]. Measurement of oxygenation using $T_{2}$ values collected with different echo times has also been demonstrated [35]. While these methods provide good agreement with other measurements of $s \mathrm{O}_{2}$, they are not typically used in clinical practice.

There are also other MR methods for detecting changes in $\mathrm{sO}_{2}$ which rely 
on the oxy-/deoxy-haemoglobin susceptibility change. The Blood Oxygenation Level Dependent (BOLD) contrast used in functional imaging relies on the susceptibility change of blood in vessels in the brain. The induced field inhomogeneity causes additional $T_{2}{ }^{*}$ relaxation, lowering the observed signal in each pixel. By tracking the signal changes over time, regions of the brain using oxygen can be identified. Measurements of phase, and susceptibility mapping technique can also be used to directly measure the susceptibility change of blood vessels $[36,37,15]$. This gives a more direct measurement of $s \mathrm{O}_{2}$, as there is no reliance on the diffusion/exchange effect and has recently been demonstrated as a potential method for calibrating the $T_{2}$ effect described above [38]. 


\section{Chapter 3}

\section{Experimental Setups}

In all experiments in this thesis, a flow circuit was used to contain the blood and provide control over parameters like oxygenation. This circuit is similar to what is used in cardio-pulmonary bypass (CPB) surgery, where a patient's blood is pumped and oxygenated by a heart lung machine. Over the course of this research, the experimental setup was improved, leading to two main versions - the original stopped flow setup, and the continuous flow setup.

\subsection{Stopped flow experimental setup}

\subsubsection{Flow circuit and pump}

A schematic of the flow stopped setup is shown in Figure 3.1. The main components are the roller pump and the oxygenation membrane, with the blood collection bag acting as a reservoir. These are all joined by $1 / 4^{\prime \prime}$ medical grade PVC tubing. The total volume of this circuit was typically $60 \mathrm{ml}$, with the blood bag containing approximately $450 \mathrm{ml}$.

The roller pump is used to generate flow around the circuit, by rotating the pump head to drive 2 rollers that squeeze the walls of the tube and force the blood along. The pump is also designed for use in CPB surgery, and is a Stockert SIII (Sorin Group, Munich Germany), with a flow rate controllable from $0.01 \mathrm{~L} / \mathrm{m}$ to $3.3 \mathrm{~L} / \mathrm{m}$ (at 250rpm) when used with the $1 / 4^{\prime \prime}$ tube. These versions of the flow circuit included a bypass section controlled by clamping the tubes so that the blood flow could be directed to around the reservoir, allowing for faster changes in the oxygenation due to a lower effective volume. One important setting on the pump is the occlusion, which controls how much the tubing is squeezed as the pump 


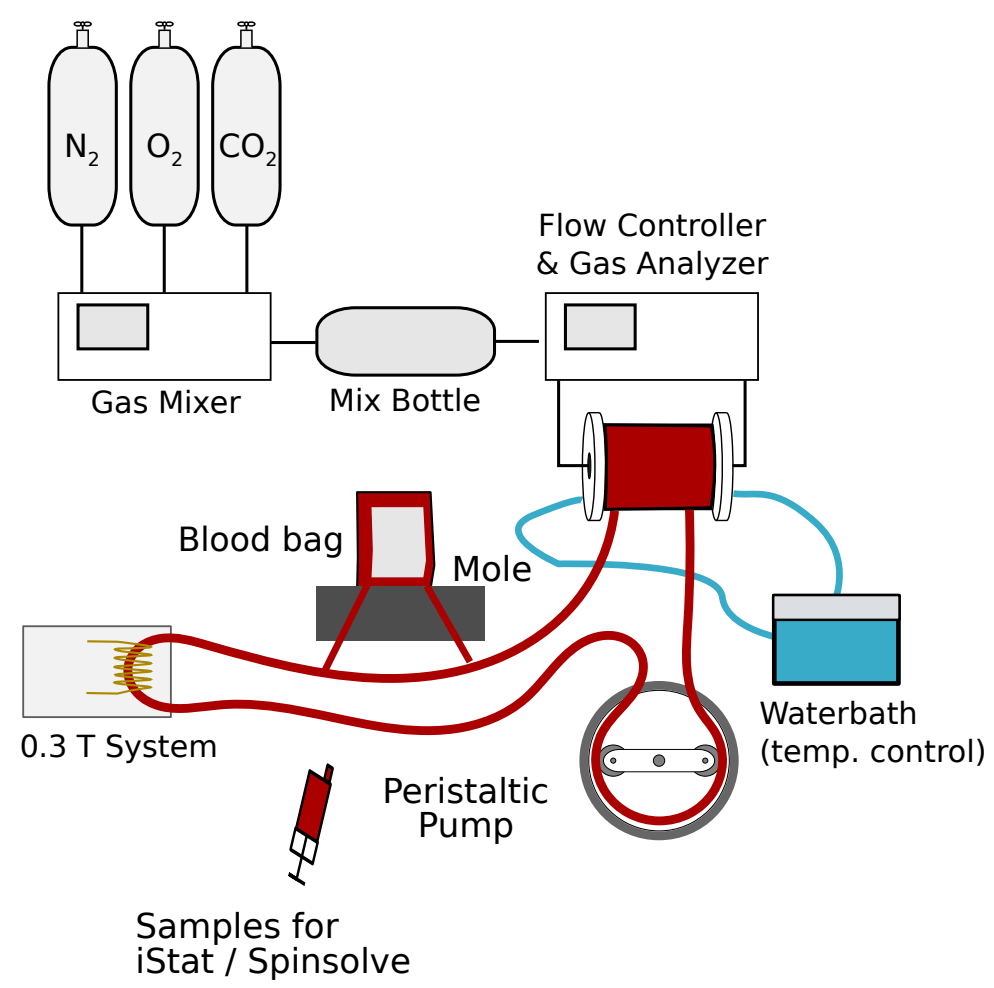

Figure 3.1: Schematic of the Stopped flow setup

head rotates. Having the occlusion set too loosely causes the pump to become ineffective, as the blood can flow backwards through the pump, while setting it too tightly was found to cause damage to the red blood cells as they travel through the pump. The model of pump used in these experiments does not have any sort of readout of the occlusion setting, so this had to be set at the correct value by following a testing procedure each time the occlusion was changed.

The occlusion testing required a piece of the $1 / 4$ " tubing in the pump, with one end going up vertically. The other end was connected to an IV bag of saline so that the water level was just above the pump. The pump head was tightened, and the pump was switched on to raise the water level in the tube, then switched off. The occlusion was then loosened until the water level started to fall, then tightened to the point where the level was stable. 


\subsubsection{Oxygenator and Gas Mixer}

A hollow-fibre membrane oxygenator was used to control the oxygenation of the blood in the circuit. This was an Affinity Pixie oxygenator (Medtronic, Minneapolis MN US), designed for use in paediatric CPB procedures. It allows for gas exchange and temperature control of the blood flowing through, with the oxygenation controlled by the mix of gases going into the oxygenator. This paediatric model was chosen to minimize the required priming volume, and it still provided more than enough capacity to oxygenate the blood at the flow rates used. The temperature of the blood was regulated by flowing water from a temperature controlled waterbath through the oxygenator, which has a separate path built into it for this purpose. The water bath temperature was set to $38^{\circ} \mathrm{C}$, although this corresponded with a blood temperature at the outlet of around $34^{\circ} \mathrm{C}$ at maximum flow rates (with lower temperatures at lower flow rates.)

The gas mix was set using a Dansensor MapMix 3 gas mixer (Dansensor, Ringsted Denmark), which allowed control of the different proportions of $\mathrm{N}_{2}, \mathrm{O}_{2}$, and $\mathrm{CO}_{2}$ flowing through the oxygenator. The Oxygen fraction was typically varied from $0 \%$ up to $21 \%$, the value of atmospheric air. Nitrogen was used as a nonOxygen containing gas, and between $2.5-5 \% \mathrm{CO}_{2}$ was also added to ensure the $\mathrm{pH}$ of the blood sample remained stable over the course of the experiment (as these are linked by the bicarbonate buffer system in blood). Gases for all experiments were used as supplied from BOC and were instrument grade or higher. The output of the gas mixer flowed into a pressurised buffer tank before flowing into a MapCheck Provectus gas analyser (Dansensor, Ringsted Denmark), which allowed us to check the fraction of the three gases which flowed into the oxygenation membrane. This gas analyser also provided some flow regulation, although this was supplemented with an adjustable flow valve. Typically, the gas flow rate was set to only $1 \mathrm{~L} / \mathrm{min}$ to $2 \mathrm{~L} / \mathrm{min}$ to avoid creating bubbles in the oxygenator.

\subsubsection{Blood collection}

Samples of whole blood were collected by venipuncture of the antecubital vein in healthy volunteers $(n=13$, median age $=23)$ at Otago medical school. This procedure was approved by the Central Health and Disability Ethics Committee (reference 15/CEN/175), and all volunteers provided written, informed consent. Approximately $450 \mathrm{ml}$ of blood was collected into a blood bag containing $66.5 \mathrm{ml}$ Citrate Phosphate Dextrose (CPD) anticoagulant/preservative solution (Leuko- 
trap WB system, Haemonetics, Braintree MA US). CPD solution is rated for storage of red blood cells for up to at least 3 weeks of storage [39]. Blood samples were held at room temperature following collection, before undergoing leukoreduction filtering to remove white blood cells, as it has been shown that the presence of white blood cells can adversely affect the condition of red blood cells in storage [39]. Samples were then moved to a refrigerator and stored at $4{ }^{\circ} \mathrm{C}$ until required for experiments (up to 30 days for stopped flow experiments, up to 10 days for continuous flow experiments).

\subsubsection{Oxygenation measurement}

The $\mathrm{sO}_{2}$ of the blood in the circuit was measured by blood gas analysis in an iStat handheld blood analyser (Abbott Point of Care, Abbott Park IL US.) This uses small sample of blood $(0.95 \mu \mathrm{l}$ for the CG8+) that is loaded into a disposable test cartridge, containing the required sensors for specific tests. The loaded cartridge is then inserted into the handheld analyser unit, to actually complete the measurements. The measurement process typically takes 2 minutes. For these experiments, the iStat CG8+ cartridge was used, which allows for measurement of $\mathrm{pO}_{2}$, $\mathrm{pH}, \mathrm{pCO}_{2}$, Haematocrit, $[\mathrm{Na}],[\mathrm{K}],[\mathrm{Ca}]$ and Glucose concentration. The $\mathrm{pO}_{2}$ is measured using a microelectrode, as described in section 2.7.

From these measurements, the iStat calculates the $\mathrm{sO}_{2}$ of the sample, based on the $\mathrm{pO}_{2}, \mathrm{pCO}_{2}$ and $\mathrm{pH}[40]$. The iStat uses the following equation, which assumes Oxygen saturation follows the normal affinity curve (Figure 2.8).

$$
s \mathrm{O}_{2}=100 \times \frac{X^{3}+150 X}{X^{2}+150 X+23400}
$$

where $\quad X=p \mathrm{O}_{2} \times 10^{0.48(p H-7.40)-0.0013([\mathrm{HCO} 3]-25)}$

While it takes into account the effect of $\mathrm{CO}_{2}$ and $\mathrm{pH}$ on the $\mathrm{pO}_{2} / \mathrm{sO}_{2}$ relationship, it does not include the effect of 2,3-DPG (diphosphoglycerate) which affects haemoglobin's oxygen affinity. It also does not take into account altered haemoglobins (e.g. met-haemoglobin). These are not expected to cause a significant decrease in the accuracy of the iStat.

\subsubsection{Temperature measurement}

The temperature of the blood flowing in the circuit was also monitored during experiments. A set of K-type thermocouples was used with a Picolog TC-08 USB 


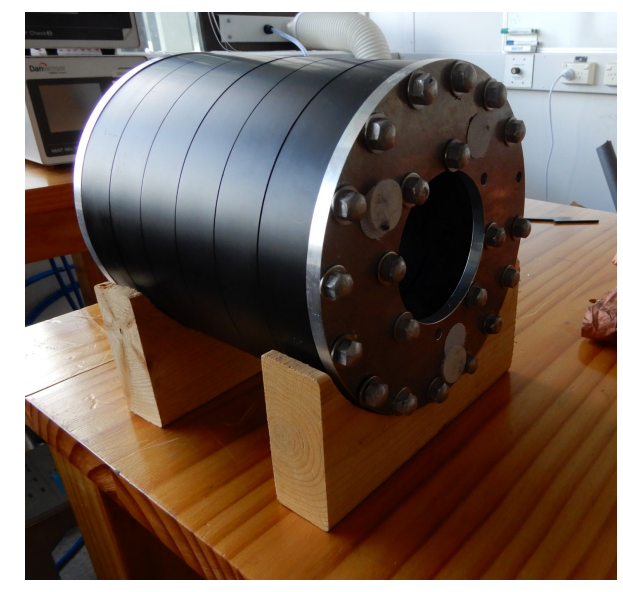

(a) Halbach magnet $(0.3 \mathrm{~T})$

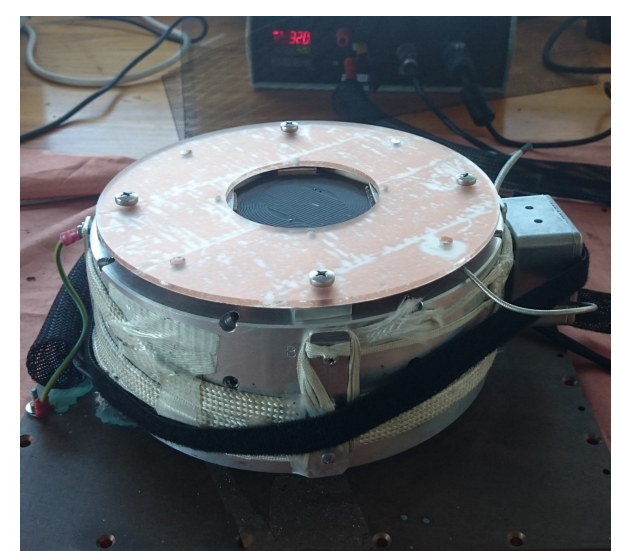

(b) NMR MOLE (0.1 T)

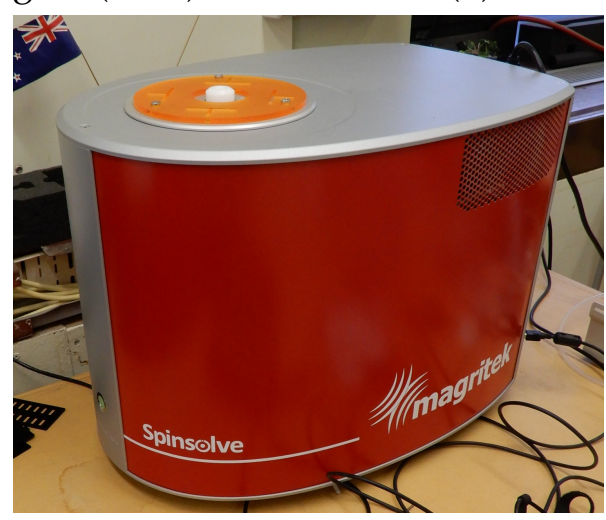

(c) Spinsolve (1 T)

Figure 3.2: Magnets used in stopped flow experiments

temperature logger (Pico Technology, St Neots UK). Temperature was measured at the oxygenator outlet, and at a spot between the two NMR systems. To maintain good thermal contact between the blood and the thermocouples, they were mounted in Luer-lock temperature connectors included in the Affinity Pixie kits. These have a hollow gold tip which extends into the tube, which the thermocouples were pressed into, allowing for temperature measurements while keeping the circuit sealed.

\subsubsection{NMR setup}

For these experiments, 3 different permanent magnet systems were used to obtain data at 3 different field strengths.

The first system was a $12 \mathrm{MHz}(0.3 \mathrm{~T})$ Halbach magnet array (Figure 3.2a), with $9 \mathrm{~cm}$ diameter bore and approximately $20 \mathrm{~cm}$ long. This magnet was repurposed 
from a previous experiment in the lab, and specifications are not available for it. When combined with the home-built coil and holder system described in subsection 3.2.3, the FID linewidth produced by the magnet was $12 \mathrm{kHz}$, which is relatively broad.

Because it uses permanent magnets, it requires a stable temperature in order to have a stable field. This was set up using a temperature-controlled water bath, which pumped water at a constant $30^{\circ} \mathrm{C}$ through tubes wrapped around the magnet. On top of this, the magnet was wrapped in a layer of foam, and a layer of mylar sheeting to insulate it from the room. To decrease the effect of electrical noise on the measurements, the magnet assembly was also surrounded by a thin copper mesh blanket (visible in stopped flow setup photo in Figure 4.2).

The second magnet system used was the NMR MOLE, previously developed by Manz et al.[41] (Figure 3.2b). The NMR MOLE operates at a field of $0.1 \mathrm{~T}$ and is a single sided device, which produces a 'sweet spot' in the region above the magnet. The sweet spot is where the combination of magnetic field and RF produced by the coil on the surface are able to create resonance, which defines the volume where signal comes from. In this case, the sweet spot was a pair of regions marking out a $3 \mathrm{~cm}$ circle over the RF coil. In the initial experiments, the tube was positioned over the sweet spot, but this tended to move during the experiment. For the last 3 experiments, the whole blood bag was placed on top of the MOLE, avoiding this issue. As with the Halbach array, the MOLE is also sensitive to temperature so an electronic temperature controller and wire heater were used to keep it stable at $29^{\circ} \mathrm{C}$.

The design of the magnet array in the MOLE creates strong magnetic field gradients across the sweet spot. This creates a wide range of resonance frequencies, and meant that it was not possible to measure an FID from the system. It was also found that this limited the possible range of CPMG echo times, as echo times longer than $1 \mathrm{~ms}$ caused excessive signal attenuation.

The third magnet system used was a Magritek Spinsolve (Magritek, Wellington NZ), which is another permanent magnet based NMR system operating at $1 \mathrm{~T}$ (Figure 3.2c). The Spinsolve is designed for chemical spectroscopy, so has a very homogeneous field and can produce a linewidth of less than $0.1 \mathrm{~Hz}$. It also uses standard $5 \mathrm{~mm}$ NMR sample tubes, so it could not be used inline in the flow circuit like the other two systems. Because of this, experiments on the Spinsolve required withdrawing a small amount of blood and transferring it into an NMR tube. This meant that that there were typically delays between removing the blood from the 
circuit and taking measurements on it, which may cause changes in the state of the blood.

Both the Halbach and MOLE systems were controlled on computers running Magritek Prospa (Magritek, Wellington NZ), and used Magritek Kea 2 (Magritek, Wellington NZ) spectrometers to run the NMR experiments. The Spinsolve was also controlled from a computer with a newer version of Prospa. CPMG experiments were run using the default CPMG macros included in Prospa, with batch scripts set up to automate taking measurements at multiple echo times.

\subsection{Continuous flow setup}

To be able to get better data on how $T_{2}$ is affected by $s_{2}$ at different fields, we decided to move to a continuous flow method, where the $\mathrm{sO}_{2}$ was slowly ramped from high to low oxygenation, while the $T_{2}$ was constantly measured. This required a number of changes to the stopped flow setup, including the use of the baby-MRI magnet, and the development of a system for continuous $\mathrm{sO}_{2}$ tracking.

\subsubsection{Flow Circuit Changes}

When first moving on to the measurements using continuous flow, the same flow circuit setup used in the stopped flow experiments was used. It was found that the design of the circuit, with the pump connected directly in line after the NMR system caused unacceptable variations in the flow rate through the probe, creating spikes in the measurements of $T_{2}$ (see subsection 5.2.2). This is due to the two roller design of the pump, which means that at a specific point in its rotation, the flow rate drops. To remedy this issue, the circuit was rearranged to include an additional empty blood bag between the magnet and the pump. A steady flow rate was generated by allowing blood to flow under gravity, with the flow regulated with a screw clamp. The screw clamp was set at the beginning of the experiment to give a flow rate of $1 \mathrm{~cm} / \mathrm{s}$ to $2 \mathrm{~cm} / \mathrm{s}$, which was measured by PGSE experiments. This gave a stable flow rate and constant $T_{2}$ measurements.

Additionally, the bypass section was removed from the circuit. It was found that portions of the blood would stagnate in this section and in the Y-joints and flow into the main circuit at random. As the oxygen saturation in the main circuit was changing, the blood in this section would lag behind and cause slugs of blood with different oxygenation to flow through the main circuit, which disrupted the gentle ramps in $\mathrm{sO}_{2}$ that were generated by the oxygenator and upper reservoir. 


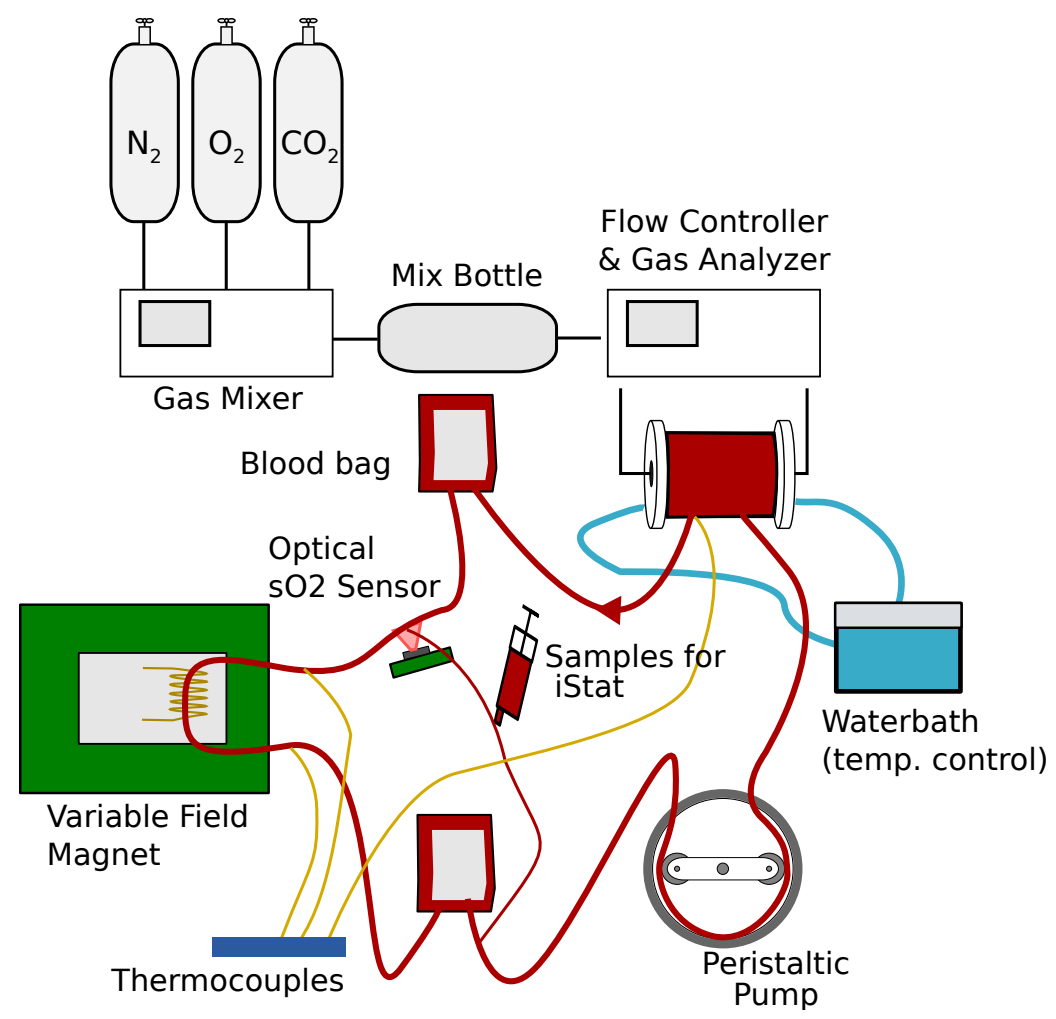

Figure 3.3: Schematic of the continuous flow circuit

The remaining parts of the flow circuit, including the oxygenator, gas mixers, and sampling methods remained the same from the stopped flow experiments. Additional thermal insulation was added to the tubing and blood bags to improve temperature stability. A diagram of the continuous flow version of the circuit is included in Figure 3.3.

\subsubsection{Baby-MRI magnet}

After the difficulties in working with the permanent magnet systems, the research moved onto the baby-MRI magnet. This is a cryogen-free magnet system (Cryogenic Limited, London UK), where the field can be changed from $0 \mathrm{~T}$ to $1.5 \mathrm{~T}$ by adjusting the current in the superconducting coils. The bore has a diameter of $8 \mathrm{~cm}$, and is $60 \mathrm{~cm}$ long. The magnetic field it generates is much more homogeneous than the permanent magnet systems, and it was possible to measure an FID with a $100 \mathrm{~Hz}$ linewidth. It also has a 3-axis gradient coil set, which allows it to be used for imaging and PGSE experiments.

The experiments were controlled by a Kea 2 NMR spectrometer (Magritek, Wellington NZ) with a gradient controller. An external RF amplifier (American 


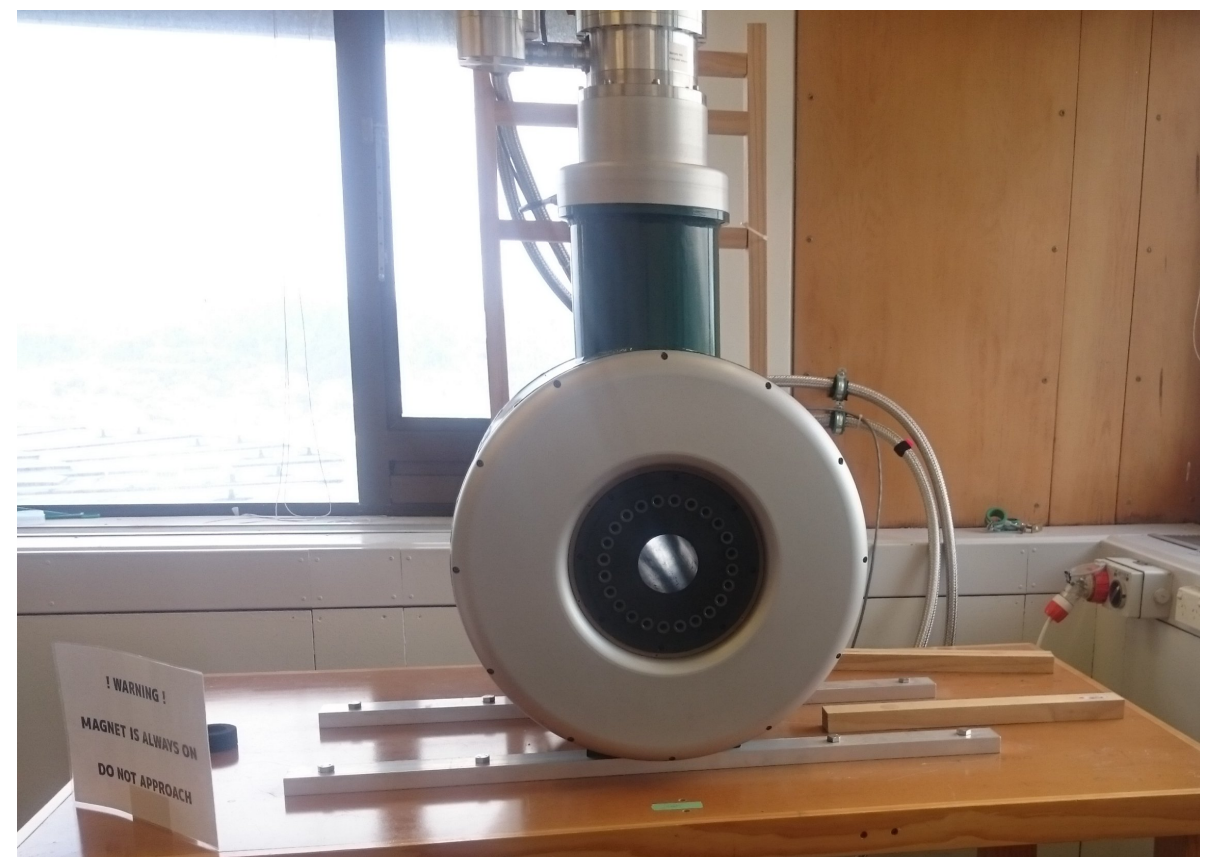

Figure 3.4: Variable field baby-MRI magnet

Microwave Technologies) is used, along with Bruker BAFPA-40 gradient power amplifiers (Bruker, Billerica, MA USA). These were used with the custom built probe, tuning and matching circuit and RF coils described in subsection 3.2.3. To help stabilise the sample temperature, the gradient set was warmed by flowing water through the cooling water tubes from a water bath set to $28^{\circ} \mathrm{C}$.

It was also discovered that the magnet power supply unit (used to change the magnet current and field strength) creates interference which disrupted NMR experiments. This occurred when echo times were approximately $8 \mathrm{~ms}$ to $12 \mathrm{~ms}$, and caused CPMG echo trains that were not exponential decays. While the exact cause of this effect is unknown, it was remedied by ensuring the magnet power supply was switched off during measurements.

\subsubsection{Coil Assemblies and Electronics}

A new coil holder, coil assembly and tuning and matching circuit was designed to fit into the baby-MRI system and also used in the Halbach magnet array experiments in chapter 4 . It was designed with exchangeable coils and tuning and matching capacitors so that the probe could be tuned and matched at multiple fields / frequencies. It consists of a probe (outer piece), which holds the coil assembly and the tuning and matching circuit inside the bore of the magnet (which had the same $8 \mathrm{~cm}$ diameter in both the Halbach and baby-MRI). 


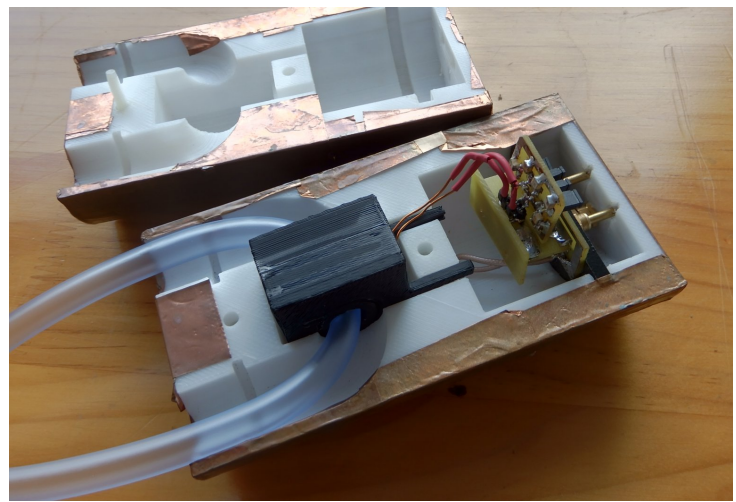

(a) Probe assembly

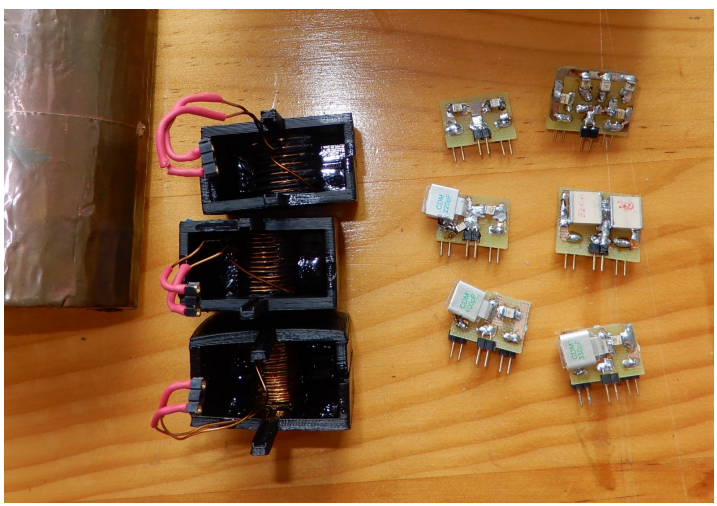

(b) Exchangeable coils and capacitor boards

Figure 3.5: Probe and Tuning and Matching equipment

The coils are hand-wound solenoids $2 \mathrm{~cm}$ long, and with a diameter of $1 \mathrm{~cm}$, so that the $1 / 4$ " tube can be passed through the coil. Different numbers of turns were used for the three different coils, to be able to use the coils at different frequencies (More turns cause more inductance, and a lower resonant frequency.) In the initial design of the coil assembly, the coil was constructed from $0.67 \mathrm{~mm}$ Copper wire wrapped around a rolled up acetate cylinder. A second version of the coil assemblies used 3D printed forms made from ABS plastic (to decrease unwanted signal), with the same wire and number of turns.

The tuning and matching circuit was also designed and produced for this probe. It allows for the capacitors to be exchanged, using small PCBs with different capacitors attached. The circuit also contains variable capacitors which are used for fine tuning the match frequency. While the higher frequencies only require small changes in capacitance to be tuned and matched using only the variable capacitors $(40 \mathrm{MHz}-60 \mathrm{MHz}$ can be tuned with $20 \mathrm{pF}$ or less from the variable capacitors), tuning and matching to lower frequencies required much higher capacitance and inductance. Switching the different capacitors allowed the probe to be tuned and matched at frequencies ranging from $2 \mathrm{MHz}$ to $60 \mathrm{MHz}$.

As the probe is located inside the magnet, the capacitors are all of a nonmagnetic type (AVX Hi-Q (AVX, Greenville SC US) or MCM series (Cornell Dubilier CDE, New Bedford MA US) and are also rated for high voltage (>500 V.)

$S_{11}$ simulations were run in Qucs Spice (qucs . sourceforge . net) to find the appropriate capacitor values $\left(C_{m} / C_{t}\right)$ to tune and match a range of frequencies with the different coils. These were then used to find the configurations in Table 3.1 for the tuning and matching circuit that were used in experiments. The configurations were tested by measuring $S_{11}$ on a spectrum analyser, before attaching to 
the Kea for wobbling.

\begin{tabular}{|c|c|c|}
\hline Frequency $(\mathrm{MHz})$ & Coil & Capacitor board $\left(\mathrm{C}_{\mathrm{m}} / \mathrm{C}_{\mathrm{t}}\right.$ in $\left.\mathrm{pF}\right)$ \\
\hline 40 & A (8 turn) & $4.7 / 1$ \\
20 & A (8 turn) & Switched $63+4.7$ \\
14 & C (10 turn) & Switched 33+18 \\
12 & C (10 turn) & Switched 63 \\
10 & C (10 turn) & $100 / 12$ \\
5.2 & E (14 turn) & $220 / 24$ \\
\hline
\end{tabular}

Table 3.1: Tuning and matching settings used in the continuous flow experiments

\subsubsection{Flow measurement by NMR}

In the continuous flow loop, the flow rate was set by adjusting a screw clamp on the tube, which meant that the flow rate could not be set directly (e.g. by setting the pump rate.) Because of this, flow measurements were done using NMR velocimetry, using the gradient coils of the baby-MRI magnet. A pulse sequence for measuring the flow rate in the circuit using a Pulsed Gradient Spin Echo (PGSE) experiment was developed, following Callaghan [10]. This pulse sequence is shown in Figure 2.6. As discussed in section 2.4, the experiment uses two gradient pulses to encode displacement into the phase of the spin echo, so that with knowledge of the phase difference and $\Delta$, the velocity of the spins can be determined. This pulse sequence was implemented in Prospa, and allows the user to set pulse parameters such as gradient direction, number of gradient steps and echo time. It can be used to measure using both the Fourier method, and the One-shot method.

To investigate variations in the flow rate, the pulse sequence also allows for a single reference measurement (where $g=0 \mathrm{~T} / \mathrm{m}$ ) followed by repetitions of the same $g$-step. This allowed for measurements of faster variations of the flow rate, when compared with the multiple gradient-step method - in this experiment the temporal resolution is set by $T_{R}$.

\subsubsection{Optical $\mathrm{sO}_{2}$ measurement}

Making measurements with continuously flowing blood required continuous monitoring of the $\mathrm{sO}_{2}$ of the blood going into the magnet system. This meant that the 


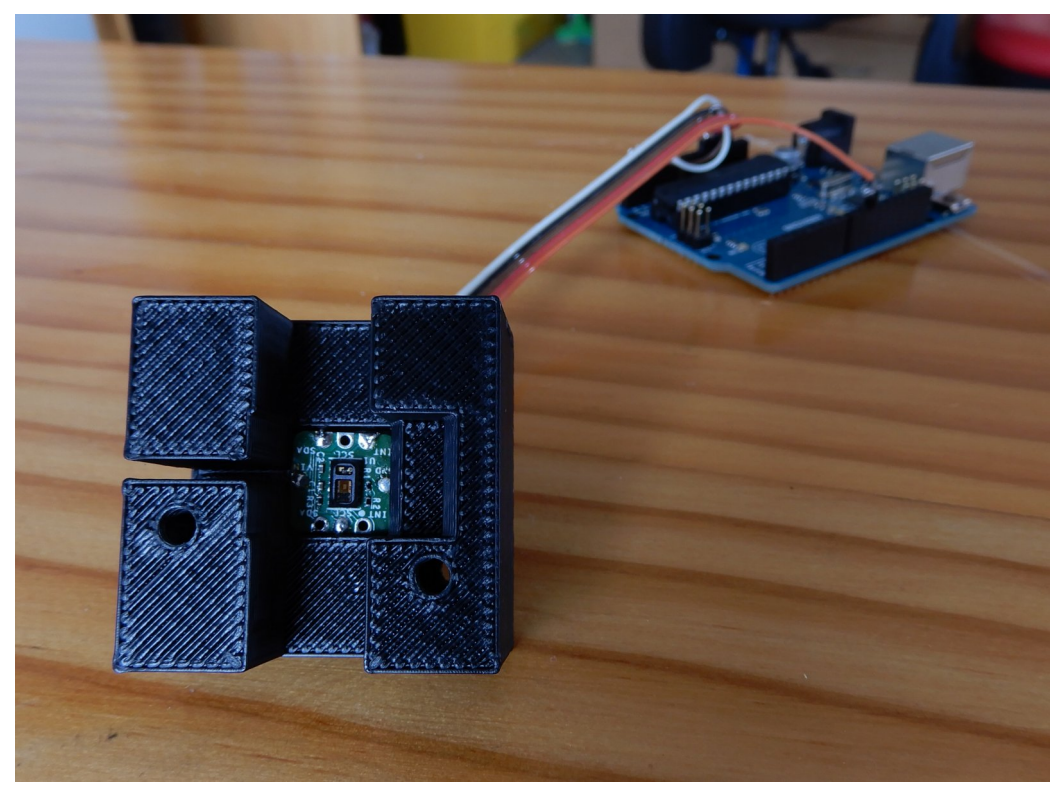

Figure 3.6: MAXREFDES117 Pulse oximeter module in 3D printed mount, connected to the Arduino.

iStat would not be suitable for these experiments, due to the length of time it took to measure each sample (approximately two minutes), and the cost of cartridges. While there were a number of possible techniques for this, such as polarographic or fibre-optic $\mathrm{pO}_{2}$ sensors, it was decided to use an optical/NIR setup.

This optical system was designed around the MAX30102 sensor (Maxim Integrated, San Jose CA US), which is an integrated pulse oximetry/heart rate module. It contains all the components for two wavelength pulse oximetry including a $660 \mathrm{~nm}$ red LED, a $880 \mathrm{~nm}$ IR LED, LED driver circuits, a photodiode and 18-bit ADC, and a digital ambient light cancellation filter. The sensor is included on an evaluation board (MAXREFDES117) (Maxim Integrated, San Jose CA US), which includes a voltage regulator and level shifter to allow it to be connected to, and supplied from $5 \mathrm{~V}$ electronics. This module was mounted in a 3D printed holder, which allowed it to be clipped onto a tubing joint used in the flow circuit, shown in Figure 3.6. It was arranged so that the sensor was shining perpendicular to a flat section of the tubing, to try and reduce any reflections from the plastic. The polystyrene plastic used in the joint did not cause significant absorption of the reflected light from the blood at either the red or IR wavelengths.

The sensor measures the red and IR light reflected by the blood by alternately pulsing the red and IR LEDs and measuring the photodiode output. In this experiment, the LED pulse time was $411 \mu$ s (the longest possible value to get the most 

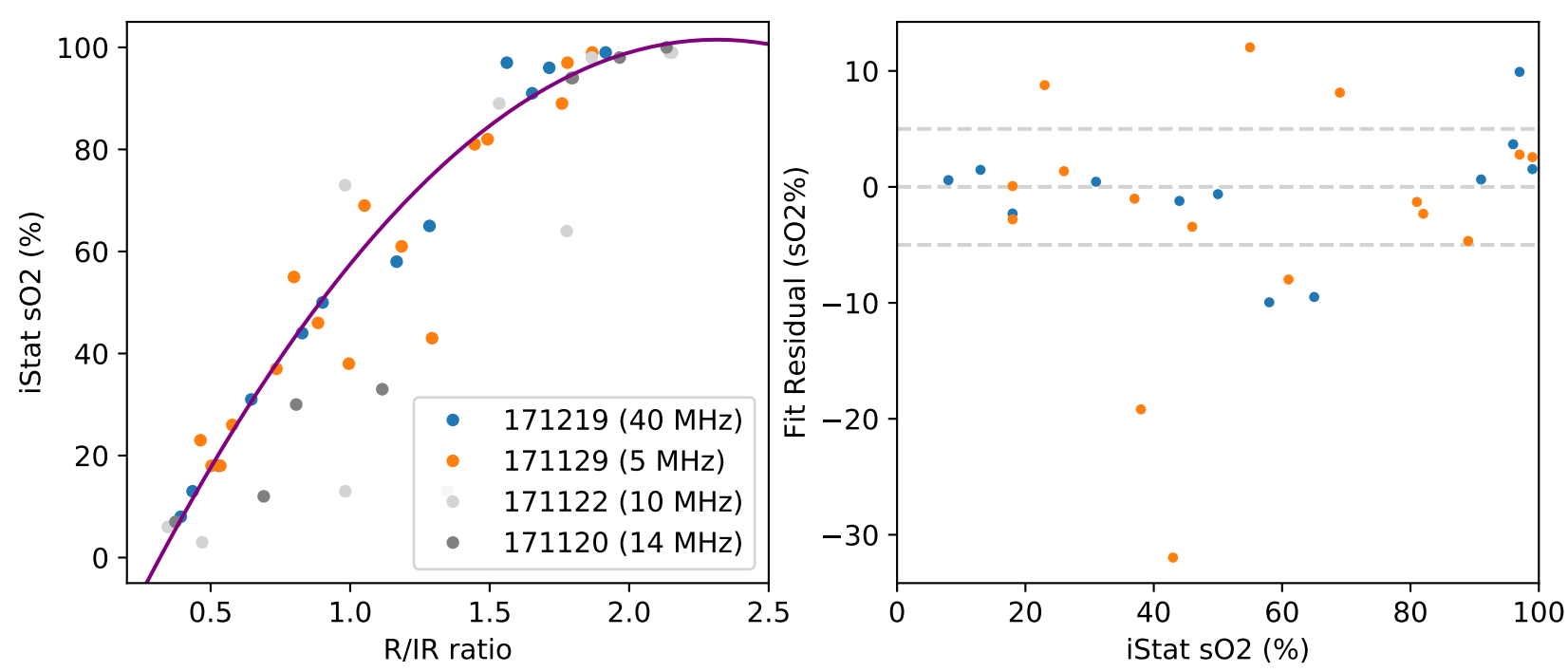

Figure 3.7: Calibration data for optical $\mathrm{sO}_{2}$ sensor. Dashed lines on residual plot show $\pm 5 \%$.

sensitivity), and the sample rate set to $100 \mathrm{~Hz}$ with a $4 \mathrm{x}$ averaging filter to give 25 samples per second. Other parameters were set to the suggested values in the evaluation demo. The MAX30102 sensor communicates over $\mathrm{I}^{2} \mathrm{C}$ to an Arduino board, that reads the incoming data and sends it to the computer over USB to be logged. This data is collected by a Python application, which generates a live plot of the data from the sensor and averages it further (100x) to reduce the amount of data stored. The application saves values of the red intensity and IR intensity every 4 seconds.

As discussed in section 2.7, the ratio of red and IR intensities is typically converted to $\mathrm{sO}_{2}$ using an empirical calibration curve. To create this curve, samples were collected while the oxygenation was being decreased, and the $\mathrm{sO}_{2} \mathrm{~S}$ measured using the iStat. The ratio for each sample was also measured, and used to fit a quadratic curve to find $s \mathrm{O}_{2}$ as a function of $\mathrm{R}$. The procedure for this process improved over the course of the experiments, to try and ensure that what was sampled from the circuit and tested on the iStat was on the sensor at the time it the ratio was measured.

To get a good distribution of data points across $R$, samples were taken every 0.1 units, approximately 2 corresponds to oxygenated blood, and 0.3 is completely deoxygenated. Before sampling, approximately $2 \mathrm{ml}$ was syringed from the sample tube (thin tube in Figure 3.3) and flushed to the other bag by syringe to remove 
the volume of the tube. A $0.5 \mathrm{ml}$ sample was taken by syringe and measured as soon as possible on the iStat. When this was not immediately possible, syringes were capped with parafilm to try and avoid any changes in $\mathrm{sO}_{2}$ due to absorbing Oxygen from the air.

Results for this calibration process from different experimental runs are shown in Figure 3.7. Because of the fast change in $\mathrm{sO}_{2}$ at intermediate oxygenations (section 2.6), it was more difficult to ensure that the blood sampled for iStat measurements hadn't changed from the blood measured at the sensor, so there is a larger scatter in the data points in the middle of the curve. This is also reflected in the fit residuals, which are typically less than $5 \%$ at high and low $\mathrm{sO}_{2}$, but increase to $10 \%$ between $50 \%$ to $70 \%$. Variations in the technique used for earlier calibration runs meant there is also more scatter for these points (points in grey in Figure 3.7). These results were used to find a calibration curve (Equation 3.1), which was used when processing experiments at all fields.

$$
s \mathrm{O}_{2}=-25.5 R^{2}+118 R-35
$$




\section{Chapter 4}

\section{Stopped flow $\mathrm{s}_{2}$ measurements}

As a first step in this research, experiments on blood using a stopped flow setup were completed, using a similar protocol and design used in previous work by the group. Stopped flow measurements are the typical method used in the literature for these blood oxygenation $T_{2}$ experiments, and there a number of examples in the literature $[19,17,18]$ (although as mentioned in the introduction, relatively few at low field). These experiments used the stopped flow experimental setup from chapter 3, with 3 different magnet systems at different fields to observe changes in $T_{2}$ at a number of levels of oxygenation. These also provide a benchmark for the continuous flow setup, as it removes any effects which occur because of flow.

\subsection{Experimental Protocol}

For this series of experiments, measurements of $T_{2}$ were made at a series of oxygenation steps, which we attempted to set by adjusting the gas mix going into the oxygenator. These steps are shown graphically in Figure 4.1 After the flow circuit was assembled, the blood sample was loaded into the tubes and oxygenator, using the standard connectors on the bag. The pump was switched on, and gas at $21 \% \mathrm{O}_{2}$ was flowed through to bring the oxygenation and temperature of the blood up to the $100 \%$ starting point for the experiments. The blood was allowed to flow through the circuit for a period of time to allow the temperature to stabilise, before an initial test with the iStat. During this time, the probes were tuned and matched, and pulse sequence parameters such as pulse power and $B_{1}$ frequency were calibrated (using FIDs on the Halbach system, and CPMG detection on the MOLE.)

If this showed that the $\mathrm{pH}, \mathrm{sO}_{2}$ and $\mathrm{HcT}$ were normal, the flow was stopped 


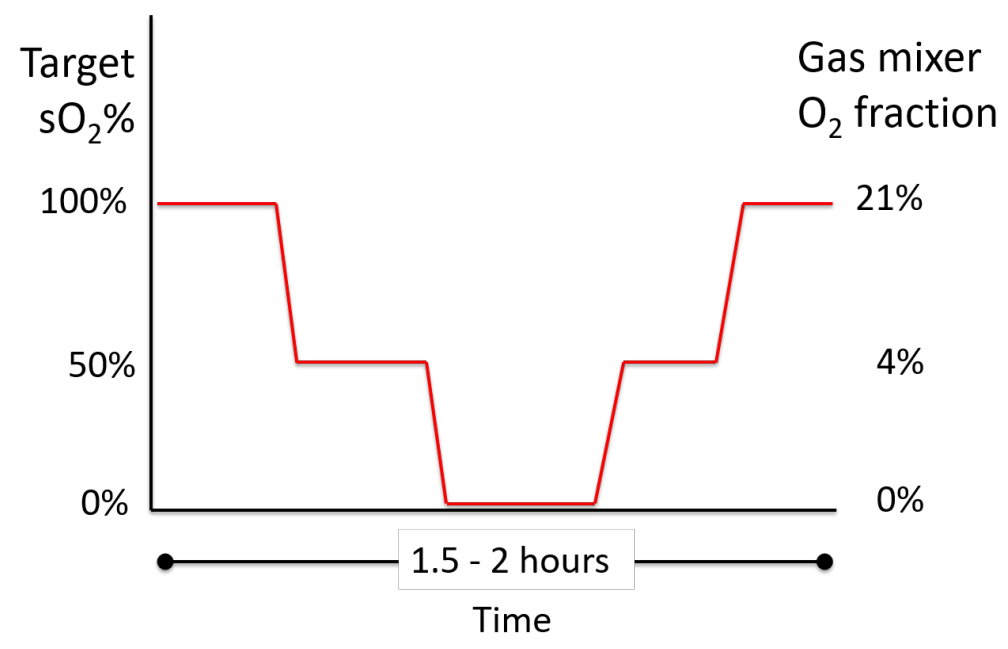

Figure 4.1: Experimental protocol for stopped flow experiments

and measurements on the two in-line NMR systems were started. If the blood $\mathrm{pH}$ was significantly below 7 , it was adjusted by adding sodium bicarbonate solution. Typically $T_{R}=2.1 \mathrm{~s}$, with echo times of $0.25 \mathrm{~ms}, 0.5 \mathrm{~ms}, 1 \mathrm{~ms}$ and $5 \mathrm{~ms}$ on the Halbach, and $0.25 \mathrm{~ms}, 0.5 \mathrm{~ms}$ and $1 \mathrm{~ms}$ on the MOLE (these were limited by the magnetic field gradients in each system). The number of echoes was adjusted to create an echo train $600 \mathrm{~ms}$ long. 4 scans with phase cycling were used on the Halbach, but the poorer signal to noise on the MOLE meant that 16 scans were required. Once these measurements had completed, the pump was turned on briefly to agitate the blood and minimize any effects of settling before repeating the NMR measurements. A $2 \mathrm{ml}$ sample of blood in the circuit was also syringed into a clean $5 \mathrm{~mm}$ NMR tube for the Spinsolve measurements. These tubes were sealed with the caps provided by the manufacturer. On the Spinsolve, typical experimental parameters were $T_{R}=20 \mathrm{~s}$ with a 4 scan phase cycle, and with 4 echo times: $0.5 \mathrm{~ms}, 2.5 \mathrm{~ms}, 5 \mathrm{~ms}$ and $10 \mathrm{~ms}$. Again, the number of echoes was set to give an echo train with a fixed length of $10 \mathrm{~s}$.

The $\mathrm{sO}_{2}$ was then lowered to an intermediate value (around 50\%). Flow was turned back on and the gas mix set to $5 \% \mathrm{O}_{2}$, which we had found produced these levels of oxygenation. After waiting for the $\mathrm{sO}_{2}$ to reach the desired level, a sample of blood was taken from the flow circuit and the iStat was used to confirm the $\mathrm{SO}_{2}$. The flow was stopped, two sets of NMR measurements were completed as above, and a sample was removed for the Spinsolve.

This process was repeated again to measure $T_{2}$ at a very low oxygenation $(10 \%$ or less), by setting the gas mix to $0 \% \mathrm{O}_{2}$. Afterwards, these steps were reversed to 


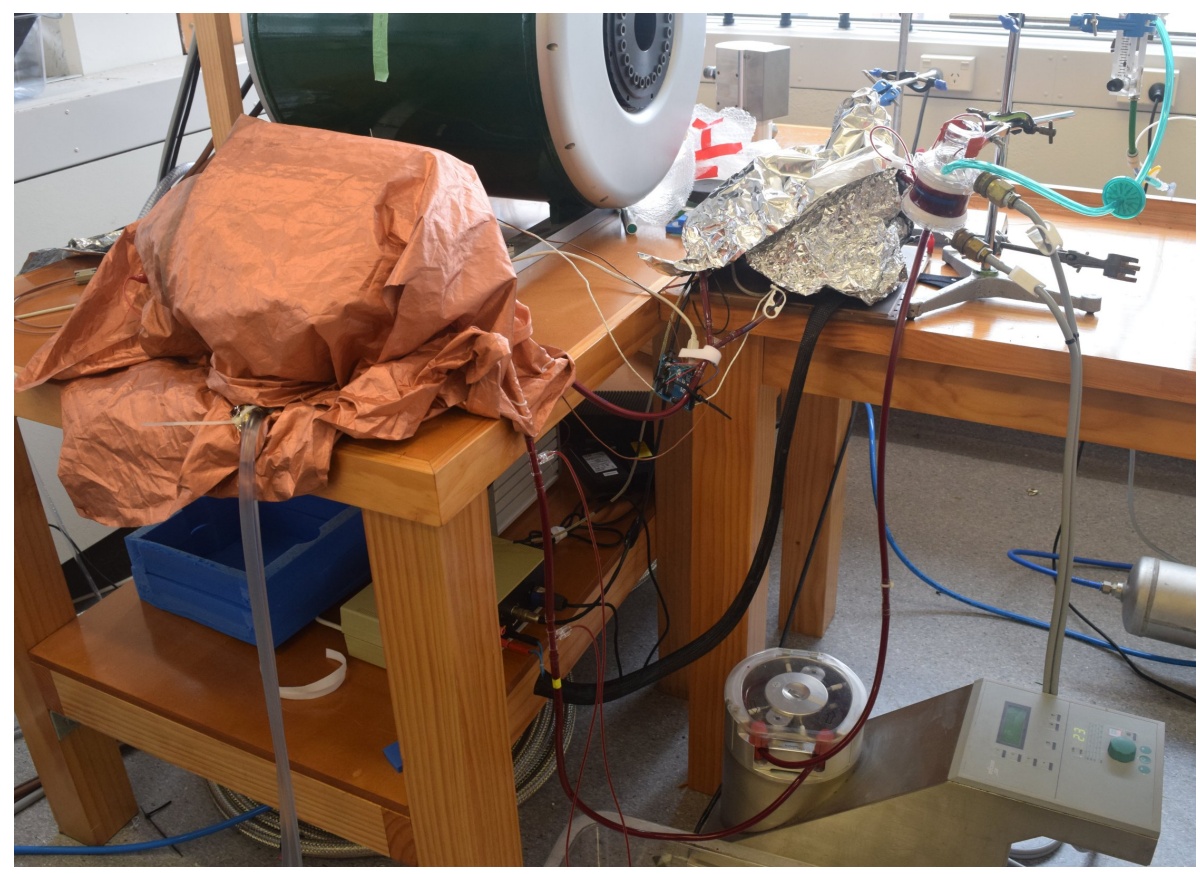

Figure 4.2: Experimental setup for stopped flow experiments (gas mixer and analyser not shown)

increase the oxygenation again, taking a measurement at an intermediate value of $s \mathrm{O}_{2}$ on the way up as well.

Data was extracted and processed using Python, in JuPyter notebooks. Each echo train is phased, and each echo is summed to give a single value for the signal at each echo time. The resulting decay is fit to a monoexponential decay using non-linear least squares fitting, which results in a single $\mathrm{T}_{2}$ value at each $\mathrm{sO}_{2}$.

\subsection{Results}

\subsubsection{Spinsolve results}

Two examples of the CPMG decays measured on the Spinsolve are shown in Figure 4.3. Plotting the log of the echo amplitudes against time gives a straight line, which indicates that the decay is mono-exponential. This agrees with what is reported in the literature.

This data also shows even-echo rephasing occurring in the decays measured with long echo times (in red). Where this was visible, only the even echoes were used in the curve fitting routine to find $T_{2}$.

Decays were measured at a range of oxygenations and fitted to obtain $T_{2}$. These 
(a) $\mathrm{sO}_{2}=95 \%$

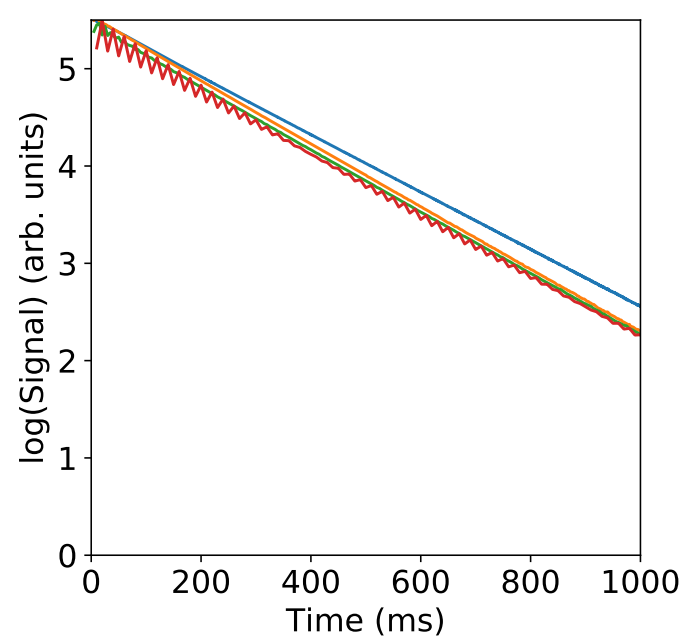

(b) $\mathrm{sO}_{2}=9 \%$

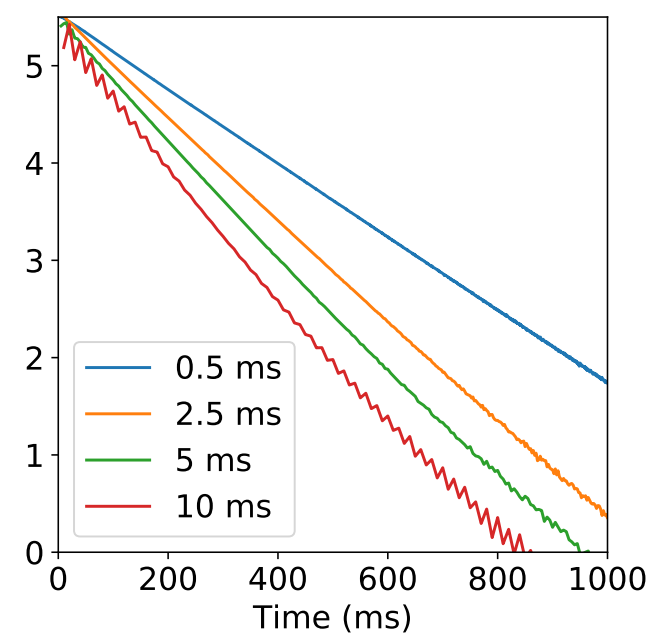

Figure 4.3: Examples of CPMG decays measured on the Spinsolve for oxygenated and deoxygenated blood

results are shown in Figure 4.4.

As the oxygenation of the blood was lowered, the $T_{2}$ decreases from $334 \mathrm{~ms}$ to $260 \mathrm{~ms}$ at the short echo time, while changes more drastically from $285 \mathrm{~ms}$ to $126 \mathrm{~ms}$ at the longest echo time. This shows that the changes in oxygenation are visible in this $1 \mathrm{~T}$ system. The shorter $T_{2}$ at long echo time is expected, as there is more time for dephasing to occur, as discussed in section 2.8. The separation between the short echo time and long echo time $\mathrm{T}_{2} \mathrm{~s}$ also increases at lower $\mathrm{sO}_{2}$. This is predicted by theory, as the susceptibility difference and induced field inhomogeneity is increased with high levels of deoxy-haemoglobin.

One difficulty we had was recovering the original $T_{2}$ value when the blood was re-oxygenated. This is shown by the two samples measured while oxygenating (triangles in Figure 4.4. A number of experiments were conducted to investigate the cause of this, and these are discussed below.

\subsubsection{Halbach results}

The Halbach magnet system was run inline with the flow circuit, making it easier to measure more data points. As with the Spinsolve results, the CPMG decays were found to be monoexponential, however there was no appearance of even echo rephasing in the CPMG decays. In this experiment, the deoxygenation causes the $T_{2}$ to decrease from $337 \mathrm{~ms}$ to $303 \mathrm{~ms}$ for the short echo time, and from 


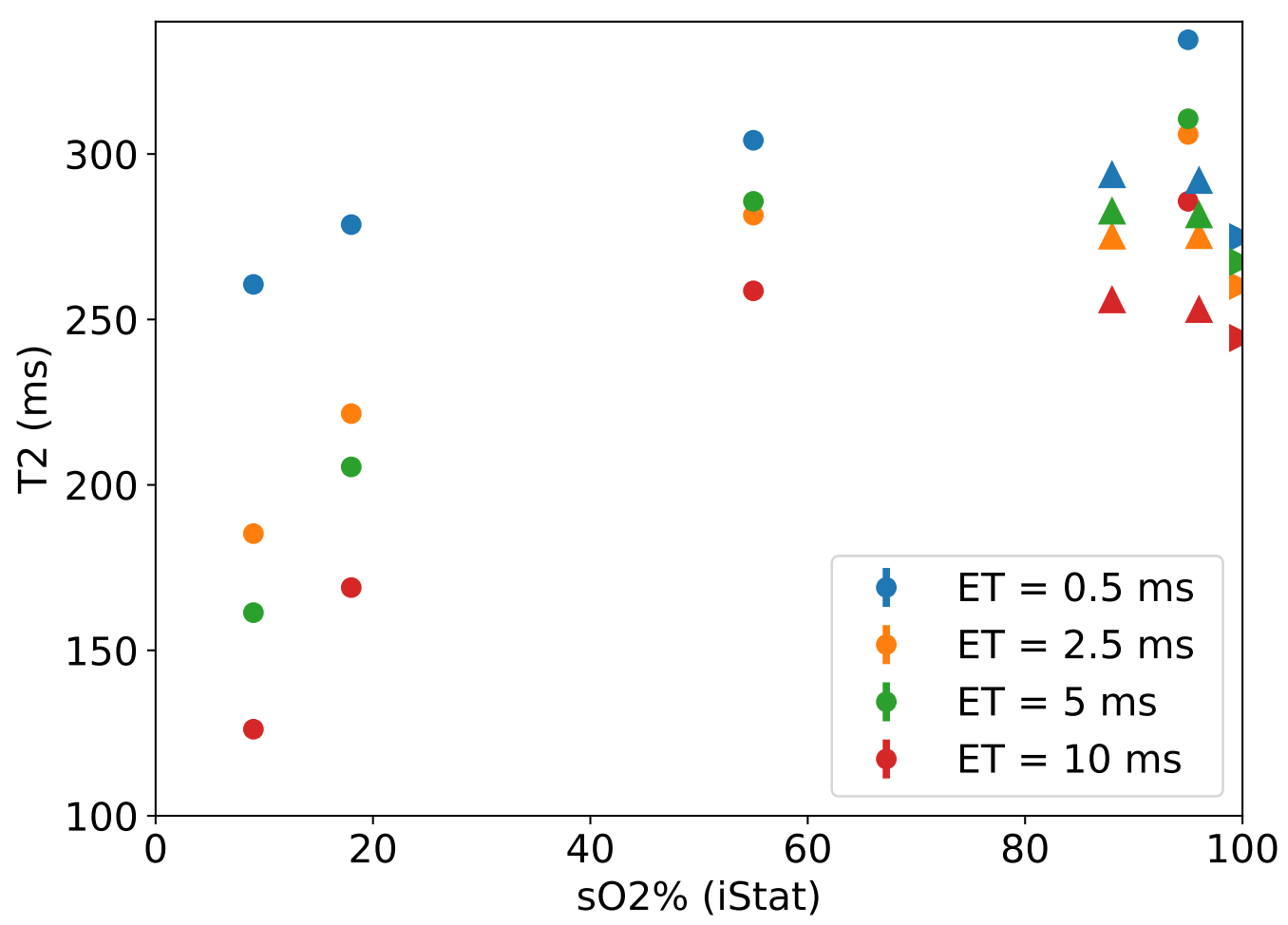

Figure 4.4: $T_{2}$ at different levels of oxygenation, measured on the Spinsolve. Circles indicate samples measured when deoxygenating the blood, while upwards pointing triangles are samples when oxygenating it (see experimental protocol). Right pointing triangles are a sample hyperoxygenated with $30 \% \mathrm{O}_{2}$

$226 \mathrm{~ms}$ to $152 \mathrm{~ms}$ for the $5 \mathrm{~ms}$ echo time. The strong field gradients in this system means that the CPMG experiments are sensitive to the diffusion of protons moving through the gradient, which causes longer echo times to measure a shorter $T_{2}$. This can be seen in the position of the $5 \mathrm{~ms}$ points (red), which are significantly lower than points at $<1 \mathrm{~ms}$. In this experiment, measurements were also completed with echo times of $8 \mathrm{~ms}$ and $12 \mathrm{~ms}$, but these did not show any trends due to the strong relaxation from the field inhomogeneities.

Like in the Spinsolve experiments, the $T_{2}$ values in this experiment did not return to the same levels they did at the start of the experiment after reoxygenating, which suggests that this change is not solely related to the sampling method and storage of the Spinsolve samples. The decrease from the initial $T_{2}$ measurement to the reoxygenated measurement appears to be approximately the same size $(40 \mathrm{~ms})$, although the decrease at the $5 \mathrm{~ms}$ echo time is much greater. This 


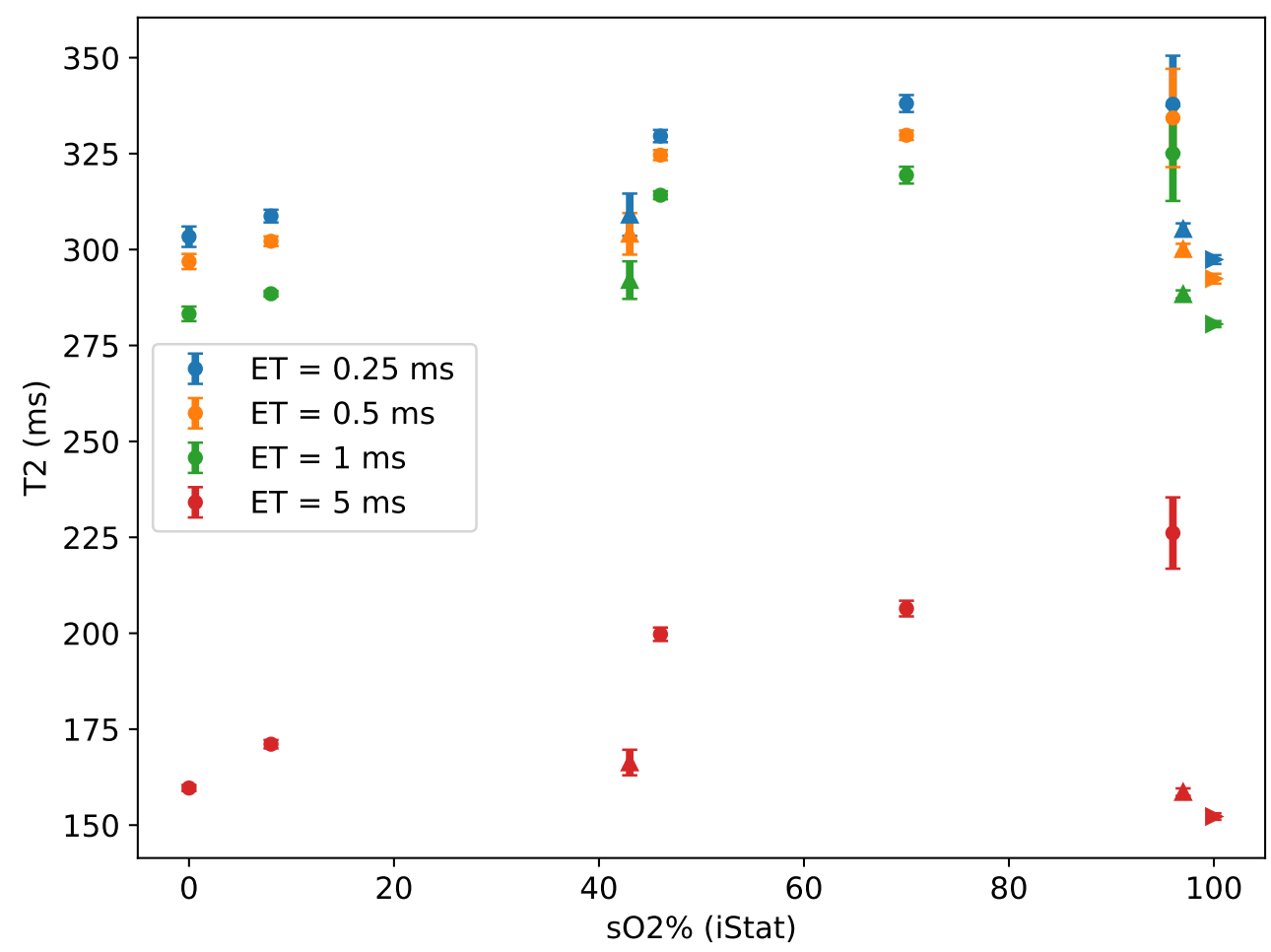

Figure 4.5: $T_{2}$ vs. $\mathrm{sO}_{2}$ measured on the Halbach system. Circles indicate samples measured when deoxygenating, upwards triangles are measured when oxygenating and right-pointing triangles are a sample oxygenated with $30 \% \mathrm{O}_{2}$. Error bars represent standard error $(n=3)$

may also be related to problems with the Halbach magnet and probe, for example, changes in magnet temperature over the experiment time or slight movements or rotations of the probe inside the magnet, which would alter the gradients seen be the sample.

\subsubsection{MOLE results}

Measurements on the MOLE had a significantly lower signal to noise than the other two systems. This is related to both the reduced field strength $(0.1 \mathrm{~T})$, and the single-sided design of the magnet system. Because of this, measurements took longer to acquire than on the other systems and the largest source of experimental uncertainty was from noise in the fitting process.

As expected, the change in $T_{2}$ as oxygenation was decreased was smaller again 


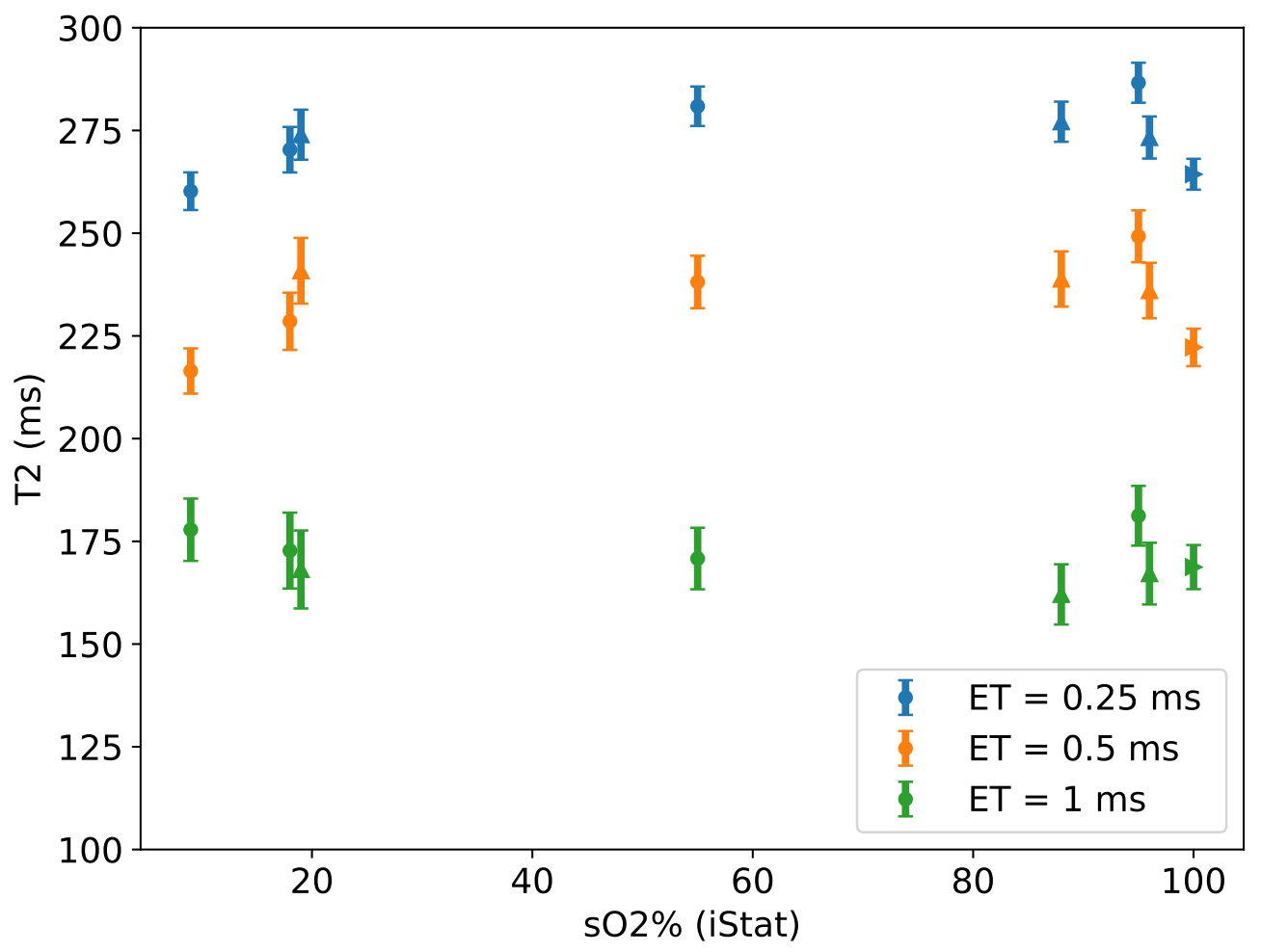

Figure 4.6: $\mathrm{T}_{2}$ vs. $\mathrm{sO}_{2}$ measured on the MOLE system. Circles indicate samples measured when deoxygenating, upwards triangles are measured when oxygenating and right-pointing triangles are a sample oxygenated with $30 \% \mathrm{O}_{2}$. Error bars represent standard error $(n=3)$

than in the higher field Spinsolve and Halbach system measurements. At the $0.25 \mathrm{~ms}$ echo time, the decrease from oxygenated to deoxygenated is $25 \mathrm{~ms}$, but at the $1 \mathrm{~ms}$ echo time, the decrease is only $5 \mathrm{~ms}$ (which is within experimental uncertainty.) Additionally, the field inhomogeneity of the MOLE means that there is a significant decrease in the measured $T_{2}$ due to diffusion through the gradients of the magnet system. Along with the decrease in the strength of the effect at this field, this could explain why there is little to no change at the $1 \mathrm{~ms}$ echo time.

These results also show that $T_{2}$ is lower after reoxygenating, with a decrease of approximately $15 \mathrm{~ms}$ between the initial $T_{2}$ and the $T_{2}$ after reoxygenation. It is unlikely to be due to changes in the magnetic field gradients as the MOLE temperature is very well controlled, and the sample was the entire blood bag, which is larger than the sweet spot of the magnet. 


\subsubsection{Field comparison}

Results for the different fields are combined in Figure 4.7, after converting the $T_{2}$ values for oxygenated and deoxygeneated blood into $R_{2}$. Although the data from the MOLE is strongly affected by its inhomogeneous field, taking the difference of the oxygenated and deoxygenated $R_{2}$ s should cancel this effect.

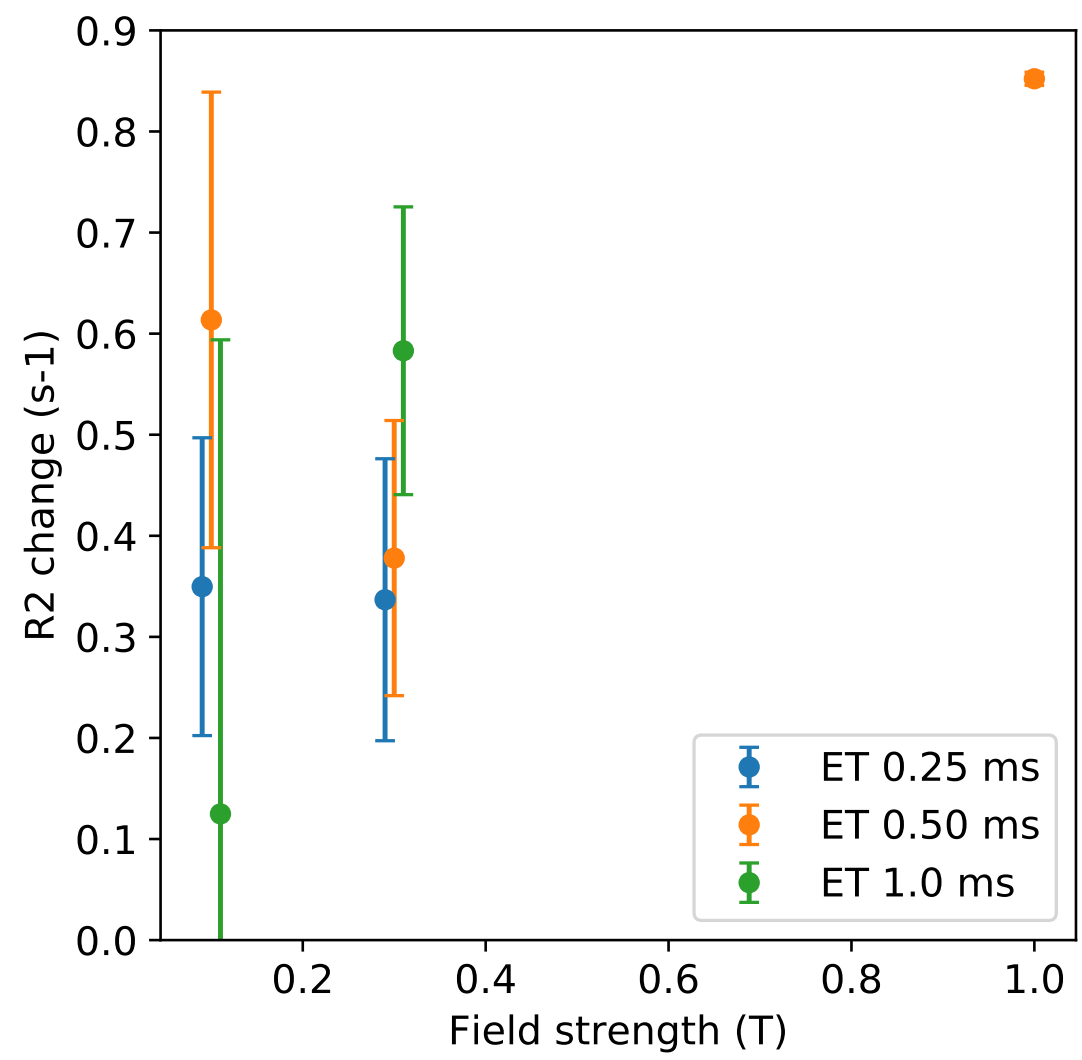

Figure 4.7: Change in $R_{2}$ for the three stopped flow systems. Data points show $R_{2}$ deoxy $-R_{2}$ oxy to remove the effect of background gradients. Note points are moved on the x-axis to allow comparison. The $1 \mathrm{~T}$ measurements used different echo times, so only the $0.5 \mathrm{~ms}$ data point is shown here

Figure 4.7 shows that the $T_{2}$ decrease is much larger at $1 \mathrm{~T}$, although data was only collected at the $0.5 \mathrm{~ms}$. Additionally, the $0.1 \mathrm{~T}$ measurement shows that the change in $R_{2}$ is higher or the same as than the data point at $0.3 \mathrm{~T}$, which does not agree with the quadratic dependence from theory, however, the uncertainty in the MOLE data points is much larger. 


\subsection{Discussions}

These results confirm that the decrease in $T_{2}$ which occurs due to decreases in $\mathrm{sO}_{2}$ is still visible at low fields, in agreement with the literature. As noted in the introduction however, there are relatively few examples in the literature to compare them to. Brooks et al. studied the field dependence of the $T_{2}$ decrease for oxygenated and deoxygenated blood, and found that the size of the $R_{2}$ change scales with $B_{0}$ squared [19, Fig.1]. For their study however, they used a more conventional $4 \mathrm{~ms}$ echo time, which makes the $R_{2}$ change from oxygenated to deoxygenated much larger (on the order of $4 \mathrm{~s}^{-1}$ at $0.5 \mathrm{~T}$.) This means that their results are not directly comparable with the results in Figure 4.7, where the echo time is less than $1 \mathrm{~ms}$, as our measurements were affected by the field homogeneity of the magnet systems.

We also encountered difficulties with recovering the initial $T_{2}$ when reoxygenating the blood. This was visible in all results above, where at high $\mathrm{sO}_{2} \mathrm{~s}$, the reoxygenated $T_{2}$ values (upwards triangles) are typically lower than the initial values (circles) by $10 \mathrm{~ms}$ to $40 \mathrm{~ms}$. In this set of experiments, several hypotheses were tested to investigate the cause of this. The fact that it occurred across all three systems (including the Spinsolve, which was used not part of the circuit) suggests that it is due to a physical change in the blood and not only related to changes in the flow circuit (although movement of the Halbach probe could not be ruled out.)

One possibility was that the blood was not completely reoxygenated, which could occur if the blood was damaged during storage or or the time at extremely low $\mathrm{sO}_{2}$, and caused the $\mathrm{sO}_{2}$ measurement on the iStat to be inaccurate. To test this, samples were hyper-oxygenated with $30 \% \mathrm{O}_{2}$ (rather than $21 \%$ ). This caused an increase in the $\mathrm{pO}_{2}$ from $110 \mathrm{mmHg}$ to $150 \mathrm{mmHg}$ in the normally reoxygenated blood to $180 \mathrm{mmHg}$ to $200 \mathrm{mmHg}$ in the hyperoxygenated blood, and should have caused more of the remaining deoxy-haemoglobin to become oxy-haemoglobin, raising the $T_{2}$. Interestingly, we observed a further decrease in $T_{2}$ in all cases, shown by the right-triangles in the results. This is not likely to be due to the paramagnetic effect of dissolved Oxygen, as this should have an extremely small effect on the $T_{2}$ at these relatively low $\mathrm{pO}_{2}$ values. Ma et al. found that dissolved Oxygen in blood plasma causes $R_{2}$ to increase by $2.3 \times 10^{-4} \mathrm{~s}^{-1} / \mathrm{mmHg}$ [42], which would correspond to a change of $0.04 \mathrm{~s}^{-1}$ at $200 \mathrm{mmHg}$.

Another possibility was that a property of the blood had changed in the circuit, with some parameter that affects $T_{2}$ being different, for example the haematocrit 


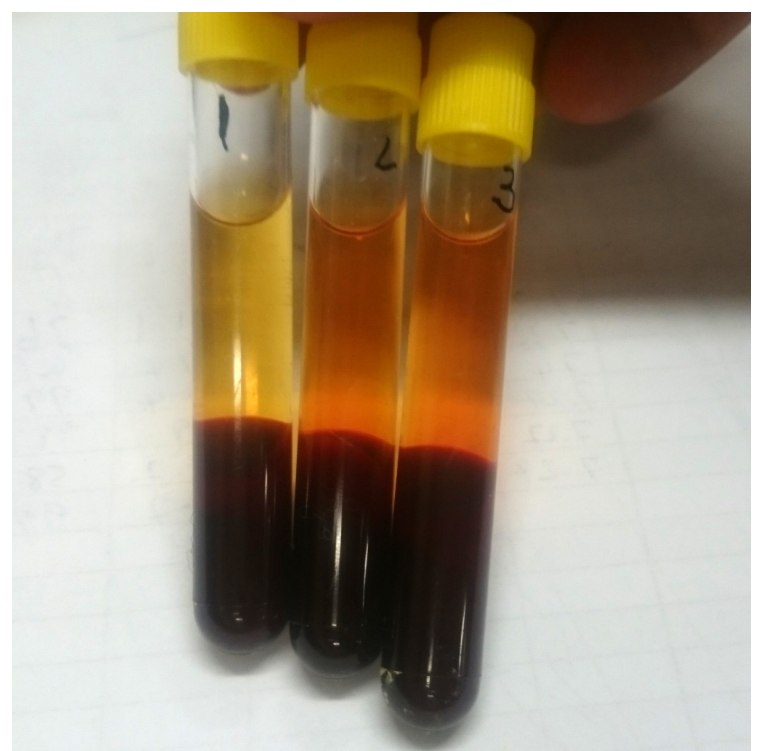

Figure 4.8: Centrifuged blood samples from before (tube 1, left), during (tube 2), and after stopped flow experiments (tube 3, right). Note the redder colour from the tubes on the right

increasing due to separation of plasma and red blood cells in the blood bag. However, testing with the iStat showed that there were not significant changes to the parameters it measured between the start and end of the experiments.

Instead, we investigated whether red blood cells were being damaged by flowing through the circuit, and that this damage caused a decrease in $T_{2}$. This would explain the $T_{2}$ decrease after hyperoxygenation, which would be caused by spending more time in the flow circuit. To test this, samples of the blood were removed from the circuit before and after the experiment. These were centrifuged to separate the red blood cells and plasma, and the colour of the plasma was compared to identify whether significant haemolysis had occured. An example of one test is included in Figure 4.8. This effect was studied more quantitatively when it occured in the continuous flow experiments described in chapter 5.

\subsection{Summary}

The results from the stopped flow experiments show that the relationship between $\mathrm{T}_{2}$ and $\mathrm{sO}_{2}$ can still be observed at low field. As expected, the $1 \mathrm{~T}$ system saw a much greater change in $T_{2}$, although changes were still visible in the $0.3 \mathrm{~T}$ and $0.1 \mathrm{~T}$ systems. This is significant, as these are representative of the field strength and homogeneities found in portable NMR devices. 
While these results confirm that changes in $\mathrm{sO}_{2}$ can be observed at low field, there were also some difficulties with this method of collecting data. As the stopped flow system requires the flow to be stopped, it is possible that the blood could be changing within the scan time due to factors such as temperature dropping, or red blood cells separating from the plasma. It was also very difficult to be able to 'hit' desired intermediate values of oxygenation. This is because of haemoglobin's strong affinity for Oxygen, which creates the very steep curve shown in Figure 2.8. To be able to reach a specific intermediate levels of oxygenation would require more precision and stability in the gas mix output than were possible with this experimental setup. Therefore, the continuous flow setup discussed in section 3.2 was designed for the next set of experiments. Because the introduction of flow adds additional complexity, these stopped flow results are used as a benchmark for the continuous flow experiments. 


\section{Chapter 5}

\section{Continuous measurements of $T_{2}$ and $s \mathrm{O}_{2}$}

The continuous flow loop experiments were designed to address some of the shortcomings of the stopped flow experiments, including the difficulties associated with measuring intermediate levels of oxygenation, and the effects of red blood cells settling when stationary. As mentioned in the introduction, measurements with continuously flowing blood have been demonstrated by Meyer [29] and the group of Peter van Zijl [43, 30, 32, 31].

The first step in this experiment was to confirm that $T_{2}$ can still be measured in a flowing sample, and to measure the effect of flow on the observed $T_{2}$. This was completed using a sample of water doped with $\mathrm{CuSO}_{4}$ to give a $T_{2}$ similar to blood. The stability of the flow rate in the circuit was also tested.

Following this, experiments with samples of blood were completed to measure $\mathrm{T}_{2}$ at a range of echo times, while $\mathrm{sO}_{2}$ was slowly ramped between oxygenated and deoxygenated. These experiments were completed at fields of $5 \mathrm{MHz}, 10 \mathrm{MHz}$, $14 \mathrm{MHz}, 20 \mathrm{MHz}$ and $40 \mathrm{MHz}$ (corresponding to approximately $0.1 \mathrm{~T}, 0.2 \mathrm{~T}, 0.3 \mathrm{~T}$, $0.5 \mathrm{~T}$ and $1.0 \mathrm{~T}$ ) to investigate the field dependence of the $T_{2}$ shortening effect.

\subsection{Experimental Protocol}

In these continuous flow experiments, the samples of blood used were 'fresher' than in the stopped flow experiments, to try and decrease any effects from haemolysis. The samples were typically used less than 3 days after collection. To prepare for the experiments, the blood was removed from the fridge and allowed to warm to room temperature while the flow circuit was assembled, and put into the mag- 
net. In most cases, the magnet was set to the correct magnetic field at least a day before (except for the $20 \mathrm{MHz}$ experiment, which was run immediately after the $40 \mathrm{MHz}$ experiment.) The water bath controlling the gradient bore temperature was also turned on, this required approximately 40 minutes to warm up and stabilise. As in the stopped flow experiments, the blood was loaded into the flow circuit, and warmed to the experiment temperature and oxygenated by flowing it through the oxygenator. During this time, the probe was tuned and matched, and NMR parameters such as pulse power and phase were calibrated. An initial sample with the iStat was measured, and a $3 \mathrm{ml}$ samples was taken for plasma separation in the centrifuge. Plasma was separated by running the centrifuge at setting ' 5 ' for 15 minutes, then removing the plasma layer by pasteur pipette.

The flow rate was set by clamping the tube between the magnet and the lower blood bag. Initially, the screw clamp was closed and the pgse-profile experiment was started. The clamp was then loosened until a flow rate around $1 \mathrm{~cm} / \mathrm{s}$ was reached, measured using the phase shift from the NMR experiment. After this was found, the screw clamp was not adjusted for the rest of the experiment, and the pump rate was set to compensate for the flow and keep a constant volume in both bags.

In these experiments the oxygenation level was slowly ramped down by flowing gas with $0 \% \mathrm{O}_{2}$ through the oxygenator while the circuit was flowing. This flowed into the upper blood bag, where mixing with the oxygenated blood helped to smooth the change in $\mathrm{sO}_{2}$ and slow down the process, so that more data points could be collected as the oxygenation decreased. In the first deoxygenation ramp at each field, samples of blood were taken for blood gas analysis on the iStat to calibrate the optical sensor, as discussed in subsection 3.2.5. This was done during deoxygenation, as we found that this had a slower rate than the oxygenation.

To oxygenate the blood, the gas mix through the oxygenator was changed to have $21 \% \mathrm{O}_{2}$. In later experiments, this was altered slightly to have a stage with $5 \%$ $\mathrm{O}_{2}$, followed by a stage at $21 \% \mathrm{O}_{2}$. This helped to slow down the oxygenation, and allow more data points to be collected. Deoxygenation and oxygenation ramps were repeated at least twice for each field/sample.

\subsubsection{NMR Experiments}

To collect the CPMG and PGSE data, a looping batch script was set up in Prospa to automatically run the experiments.

Multiple versions of the standard CPMG experiment included in Prospa were 


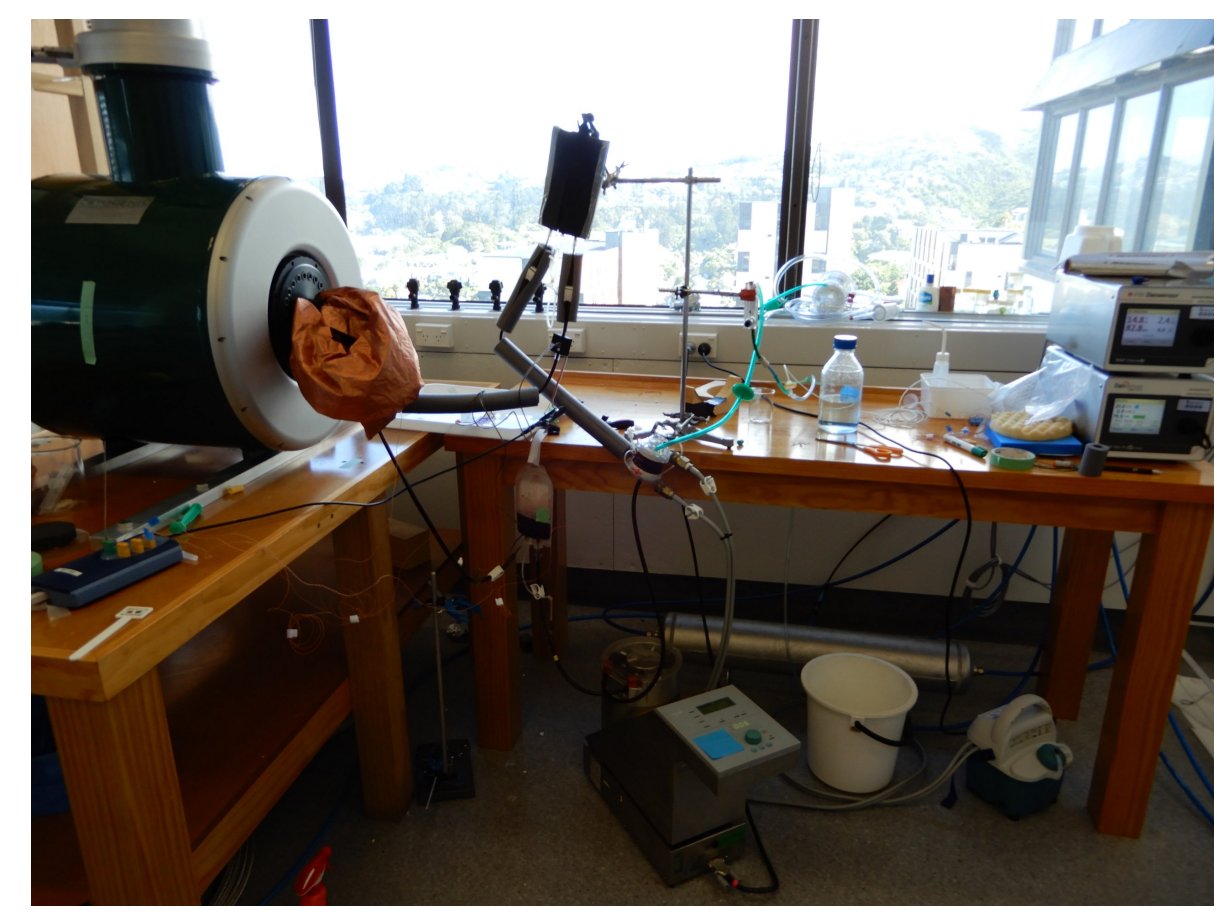

Figure 5.1: Experimental setup for continuous flow experiments (gas mixer and analyser not shown)

added with echo times equal to $1 \mathrm{~ms}, 5 \mathrm{~ms}, 8 \mathrm{~ms}, 10 \mathrm{~ms}$ and $20 \mathrm{~ms}$, and the number of echoes varied to give a total echo train length of $600 \mathrm{~ms}$. 4 scans of each CPMG experiment were used with the standard phase cycling and a $1.2 \mathrm{~s}$ interexperiment delay (the sample was flowing through the coil, bringing in 'fresh' spins, so the repetition time could be less than $5 \times T_{1}$.) Additionally, a PGSE experiment was added to monitor the flow rate, with $\Delta=16 \mathrm{~ms}, \delta=2 \mathrm{~ms}, g=$ $0 \mathrm{~T} / \mathrm{m}$ to $0.015 \mathrm{~T} / \mathrm{m}$ over 4 steps, and a $15.5 \mathrm{~ms}$ echo time. The $x$ gradients were used as the PGSE gradient, as this corresponds to the direction of flow through the coil. Equation 2.8 (restated here) was used to calculate the flow velocity.

$$
v=\frac{\phi}{\gamma \Delta \delta g}
$$

These parameters allow for measurement of velocities $<5 \mathrm{~cm} / \mathrm{s}$ without phase wrapping. A standard 1pulse FID experiment is also run, to test for any shifts in the magnetic field.

The batch script in Prospa runs each experiment, then moves to a new directory and repeats. Each cycle of 7 experiments takes approximately 45 seconds to complete. Data is also continuously collected from the optical $\mathrm{sO}_{2}$ sensor, and the Picolog temperature logger. 
The data is processed using Python in JuPyter notebooks. Each echo train is phased, and each echo is summed to give a single value for the signal at each echo time. As in the stopped flow experiments, signal before $15 \mathrm{~ms}$ was ignored in the fitting process to remove the signal from the tube. To try and minimise the effect of flow, the section of the decay used to find $T_{2}$ was also limited to the first $360 \mathrm{~ms}$ of the echo train. The $T_{2}$ and fitting uncertainty was then found by nonlinear exponential fitting of the echo train. Additionally, the time of each CPMG experiment was extracted from the file system, so that it could be compared with the $\mathrm{sO}_{2}$ measurements.

\subsection{Flow testing}

\subsection{1 $T_{2}$ and flow}

Having the sample flow through the coil causes a decrease in the measured $T_{2}$, due to the effect of added dispersion, and the outflow of excited spins from the coil. In particular, the outflow effect becomes significant as the time in the coil ( $2 \mathrm{~cm}$ long) becomes shorter than the experiment time, as the decrease in spins from the coil adds to the true $T_{2}$ decay and causes the $T_{2}$ to appear shorter. To test the impact of flow on the $T_{2}$ measurements in this experiment, a $1 \% \mathrm{NaCl}$ solution doped with $5 \mathrm{mmolL}^{-1} \mathrm{CuSO}_{4}$, was prepared to test with. This was loaded into a circuit without the oxygenator and CPMG and PGSE experiments were measured at a range of flow rates.

Figure 5.2 shows CPMG echo trains measured for the doped water measured at 4 flow rates, shown on a log scale. In the first $100 \mathrm{~ms}$, all four decays appear the same, but as the flow rate increases, the lines become curved as the signal stops being monoexponential. As expected, this effect is much larger at the highest flow rate $(4.6 \mathrm{~cm} / \mathrm{s}$.) Interestingly, this speed corresponds with a time in the coil of only $430 \mathrm{~ms}$, but signal is still being detected after this, suggesting that there is a distribution of speeds in the sample. There appears to be little effect at the two lower flow rates, and at $2.2 \mathrm{~cm} / \mathrm{s}$, the effect is not significant until approximately $300 \mathrm{~ms}$ into the decay. These decays were fitted with non-linear fitting to obtain $T_{2}$ values at different flow rates, shown in Figure 5.3.

As expected, the $T_{2}$ values measured at higher flow rates decrease from $174 \mathrm{~ms}$ to around $150 \mathrm{~ms}$, with a stronger effect measured at the $20 \mathrm{~ms}$ echo time. The decrease appears roughly linear for all but the fastest speed, and these data points were fitted to determine the trend, and the intercept where $v=0$. These fit param- 


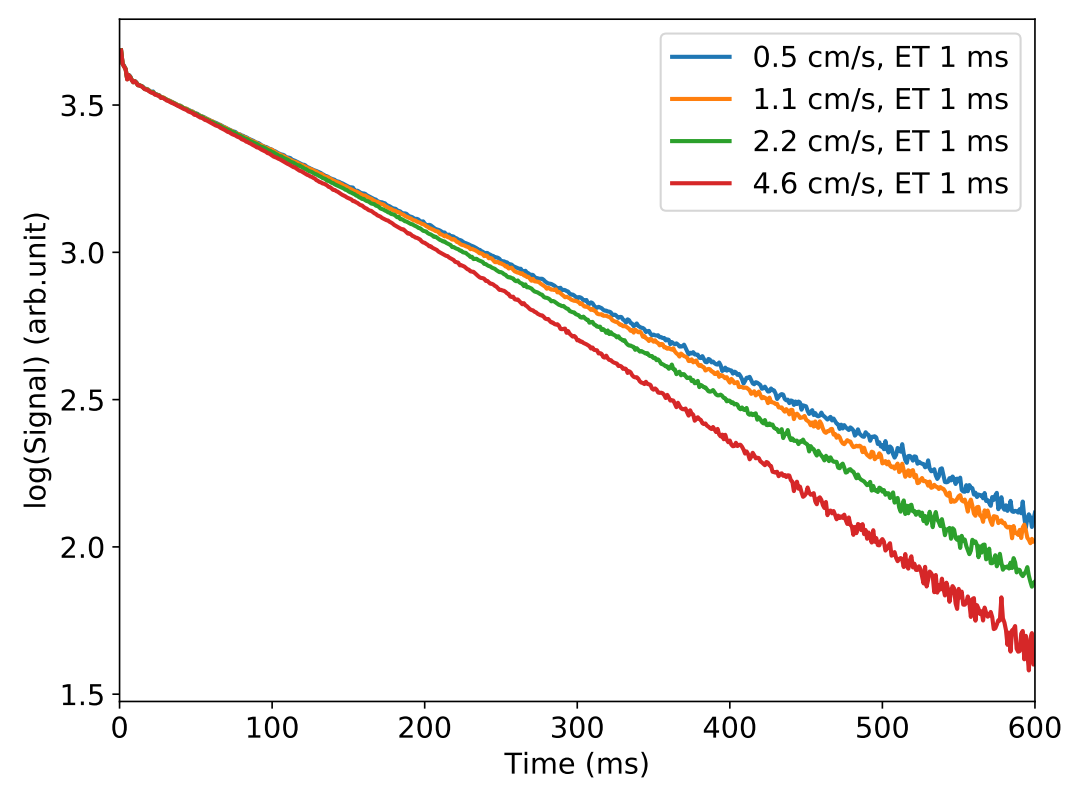

Figure 5.2: Examples of CPMG echo trains for doped water samples at different flow rates

eters are included in Table 5.1.

Because the $T_{2}$ decrease due to flow becomes negligible at low speeds (as the time in coil increases proportional to $\frac{1}{v}$ ), the intercepts of the lines of best fit overestimate the true $T_{2}$ (174 ms, measured in a separate experiment) by $4 \mathrm{~ms}$. Additionally, the slopes would be dependent on the true $T_{2}$ of the sample, as a sample with a short $T_{2}$ would be less affected by the effect of flow, and vice versa. As the $T_{2}$ of the blood will be changing during the experiment, applying the linear correction shown here would not necessarily work. However, from these experiments, the change in $T_{2}$ due to flow should be less than $10 \mathrm{~ms}$, for flow rates less than $1.5 \mathrm{~cm} / \mathrm{s}$. In blood $T_{2}$ changes due to blood oxygenation are larger than this effect - on the order of $50 \mathrm{~ms}$ at $10 \mathrm{MHz}$ - and the flow rate should stay relatively constant over the course of the experiment, so effects from flow should be relatively small.

\subsubsection{Flow stability}

The stability of the flow produced in the circuit was also tested, after early experiments showed a series of spikes in the $T_{2}$ data.

An example is shown in Figure 5.4, from an experiment with blood at $14 \mathrm{MHz}$. 


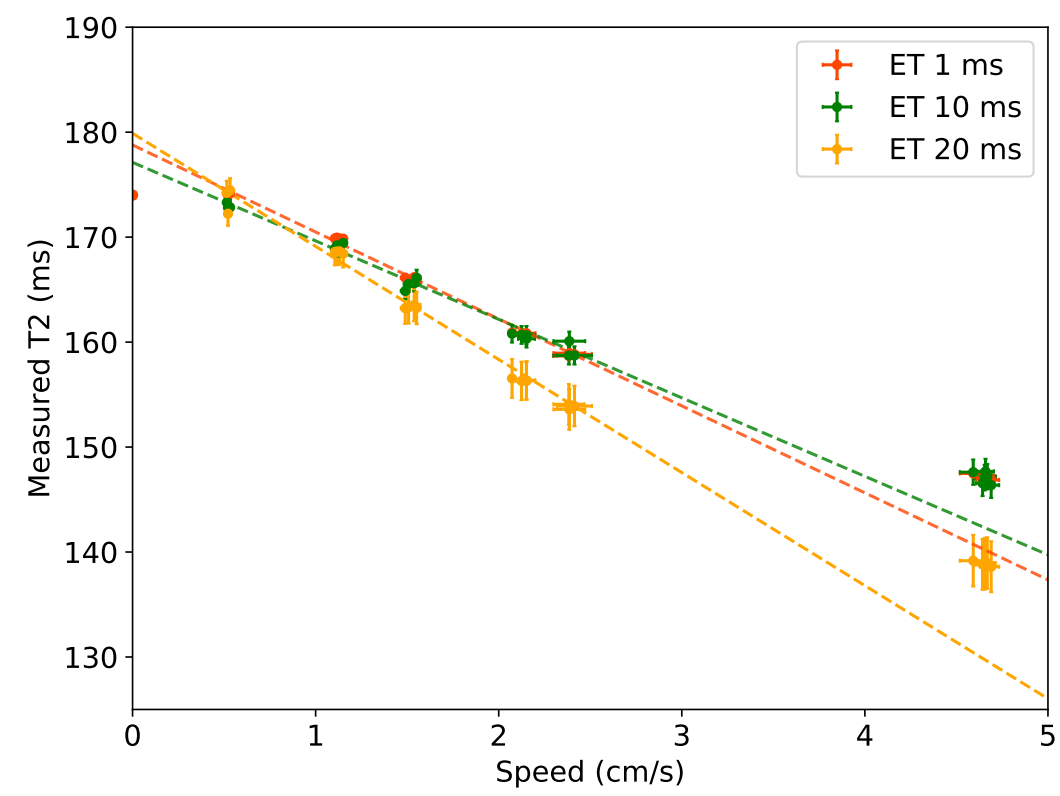

Figure 5.3: Measured $T_{2}$ values at different flow rates. $v=0$ point measured in separate experiment. Lines fitted to points where $\mathrm{v}<3 \mathrm{~cm} / \mathrm{s}$.

Table 5.1: Measured $T_{2}$ dependence on speed

\begin{tabular}{|c|cc|}
\hline Echotime $(\mathrm{ms})$ & Intercept $(\mathrm{ms})$ & Slope $(\mathrm{ms} / \mathrm{cm} / \mathrm{s})$ \\
\hline 1 & $178 \pm 1$ & $-8.2 \pm 0.2$ \\
10 & $177 \pm 1$ & $-7.4 \pm 0.3$ \\
20 & $179 \pm 1$ & $-10.7 \pm 0.3$ \\
\hline
\end{tabular}

There are large spikes of up to $40 \mathrm{~ms}$ in the measured $T_{2} \mathrm{~s}$, which makes it difficult to see trends due to changing $\mathrm{sO}_{2}$. Interestingly, there is a period of 'calm' between 355 and 380 minutes, before it goes back to the random spike pattern.

There were a number of possible causes for this effect, including absolute phase effects from the timing in the Kea spectrometer and changing sources of RF noise. This was eventually tracked down to an issue with the stability of the flow in the circuit. In these experiments, the lower bag was not included in the circuit, so that the magnet output was connected directly to the peristaltic pump. To see how this affected the flow circuit, PGSE experiments were used to measure the velocity of doped water sample flowing in the circuit.

Figure 5.5 shows the raw spin echoes collected in these experiments. In this experiment, a constant value of $g=0.01 \mathrm{~T} / \mathrm{m}$ was used so that changes in phase 


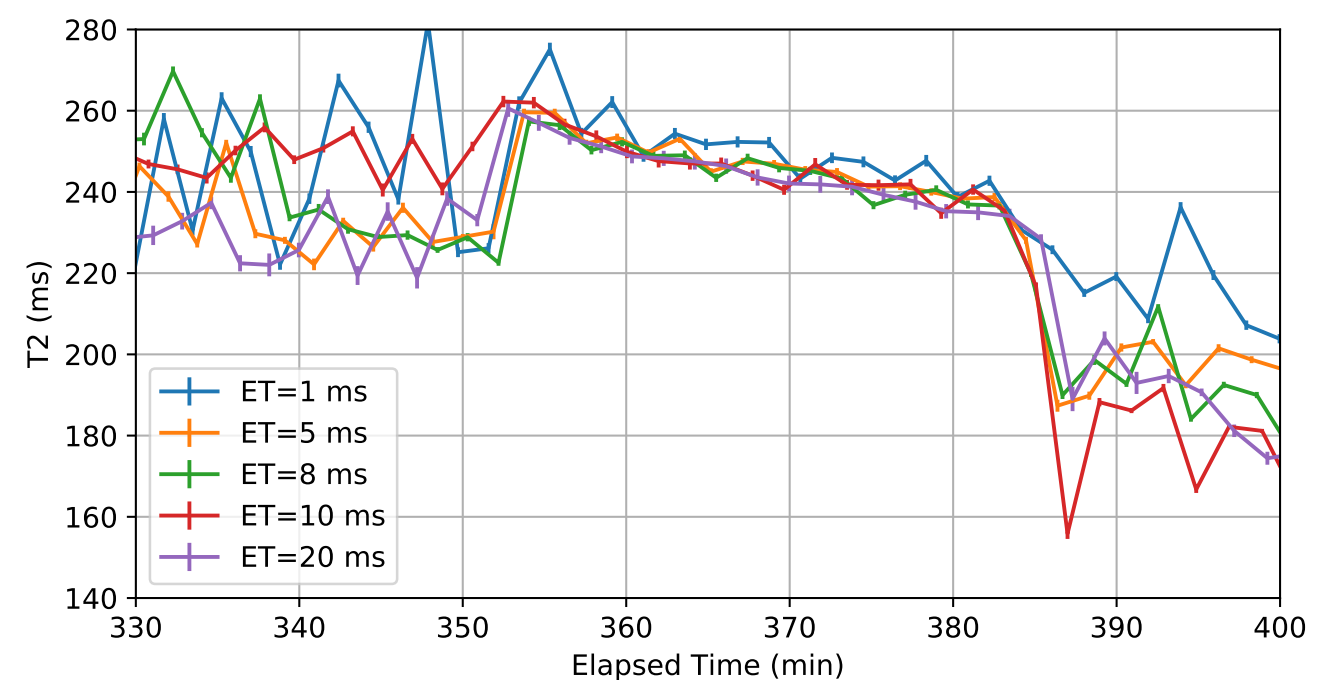

Figure 5.4: Spikes in continuous $T_{2}$ measurements from unstable flow

reflect changes in the velocity. The experiment with no flow shows no changes in phase, as expected. However, when the pump is turned on, there are clearly disturbances, which occur with a period of 13 seconds. Processing this to find the velocities gives Figure 5.6, which shows that the flow rate from the pump drops by over $50 \%$ every 13 seconds. This is half of the rotational period of the pump head (approximately 26 seconds when set to $2.3 \mathrm{rpm}$ ), which is expected as it has two rollers. This drop is from a short period of time where both rollers stop squeezing the tube as the head rotates. When this is combined with the effect of phase cycling, this could cause the spikes in the measured $T_{2}$.

The flow circuit was modified to include another blood bag between the magnet and the pump, so the blood flowed under gravity. This give a much more stable flow, as can be seen in Figure 5.5(c), and the green curve in Figure 5.6, which has a speed of $v=(1.04 \pm 0.02) \mathrm{cm} / \mathrm{s}$ over the 100 seconds it was measured for.

\subsection{Blood results}

One sample of blood was used to do measurements at each field strength. These results are shown as plots of $T_{2}$ against the experiment time, which was started when the blood sample was loaded into the circuit. The calibrated $\mathrm{sO}_{2}$ measurements from the optical sensor as also shown on the same plots. No correction is made to this data to adjust for the distance/delay between the oxygen sensor and the NMR probe, which was typically 1-1.2 minutes. Additionally, the flow rate 
(a) No flow

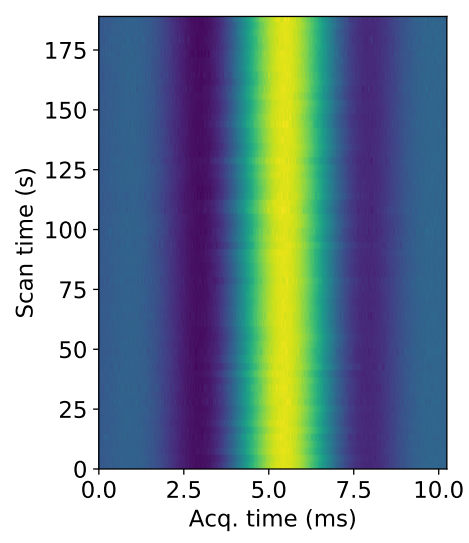

(b) Pump flow

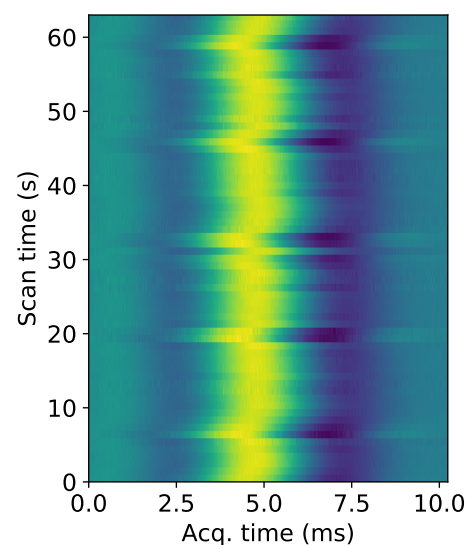

(c) Gravity flow

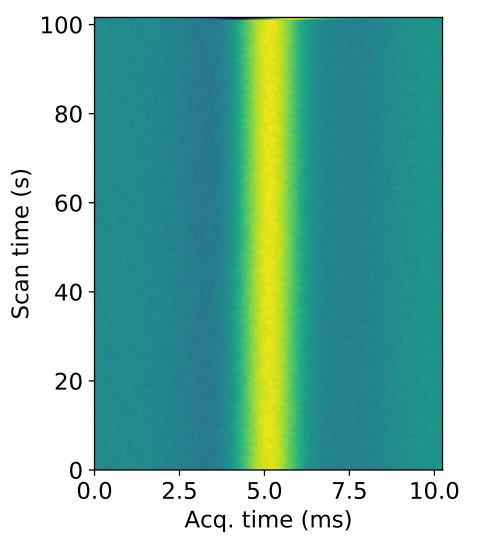

Figure 5.5: PGSE data showing stability for different flow setups. Collected with PGSE experiments using a repeated $g / q$ step so phase changes show velocity. The $\mathrm{x}$-axis shows the acquisition time, while the experiment time is on $\mathrm{y}$. Colour shows the intensity of the real part of the signal.

was monitored during the experiments but was not used to adjust the $T_{2}$ values. Temperature was also measured during each experiment, and generally stayed within $(29 \pm 4){ }^{\circ} \mathrm{C}$ (temperature control was better in some experiments, this is shown in the captions).

\subsection{1 $\quad 40 \mathrm{MHz}$}

The blood used in the $40 \mathrm{MHz}$ and $20 \mathrm{MHz}$ experiments was collected the day before the experiment. After the initial temperature stabilising section, the $\mathrm{pH}$ was 7.32 and haematocrit was 0.35. The results are shown in Figure 5.7.

At a field of $40 \mathrm{MHz}$ (approximately $1 \mathrm{~T}$ ), there is a clear drop in $T_{2}$ from when the oxygen saturation is lowered. The initial $T_{2}$ is around $350 \mathrm{~ms}$, which is quite high compared to the stopped flow results (Spinsolve at $42 \mathrm{MHz}$ measured $310 \mathrm{~ms}$ for initial $T_{2}$. There is also a $40 \mathrm{~ms}$ splitting between the short and long echo times, which should be much smaller at this oxygenation. This could be due to factors such as magnet shimming, or dispersion from flow.

As the $\mathrm{sO}_{2}$ is lowered, around 90 minutes, there are spikes visible in both the $\mathrm{T}_{2}$ and $\mathrm{sO}_{2}$. These are due to samples of blood being syringed out of the circuit to calibrate the optical sensor. The splitting between the short and long echo times also increases as the blood is deoxygenated.

The blood was assumed to be deoxygenated at 120 minutes. Here, the split- 


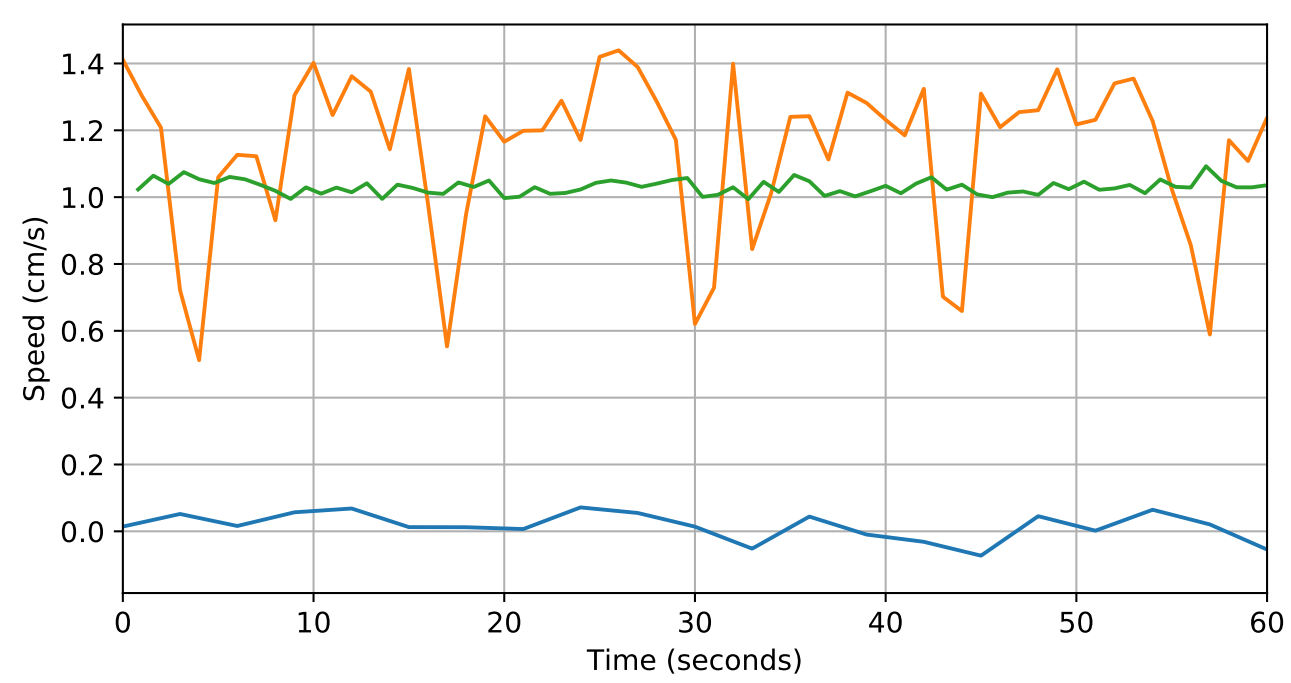

Figure 5.6: Flow rates measured by PGSE for: No flow (blue), Pump flow (yellow), Gravity flow (green)

ting between the echo times becomes much clearer - the $1 \mathrm{~ms}$ echo time has a $T_{2}$ of $250 \mathrm{~ms}$, while the $20 \mathrm{~ms}$ echo time is at $75 \mathrm{~ms}$. This agrees with the trends seen in the stopped flow results, although the actual $T_{2}$ values is higher in this experiment. The gap from minute 110-121 (and also 184-205) was due to stopping the looping batch file and running the experiments discussed in chapter 6 .

The $\mathrm{sO}_{2}$ was then increased again, with a stage at $5 \% \mathrm{O}_{2}$ followed by a stage at $21 \%$. The $T_{2}$ increases to around $280 \mathrm{~ms}$ when reoxygenated (at 181 minutes), rather than the initial $350 \mathrm{~ms}$ value. This effect was also seen in the stopped flow experiments.

During the second deoxygenation ramp, the top blood bag ran empty due to the pump being set too slow (minute 225). This stopped the smooth deoxygenation ramp, and meant that the relatively deoxygenated blood from the oxygenator was sent through the circuit without mixing with oxygenated blood in the bag. This can be seen in the optical data, as there is a sudden drop to around $30 \%$ saturated, and it then stays constant. The $T_{2}$ responds differently however, with a slight increase, followed by the expected decrease. This may be due to the deoxygenated blood mixing with the oxygenated blood in the tube as it travels from the optical sensor to the magnet. The $T_{2}$ values measured at the second deoxygenated stage (260 minutes) agree well with values in the first deoxygenated stage, although there is a spike in the $1 \mathrm{~ms}$ echo time data which does not appear in the others. 

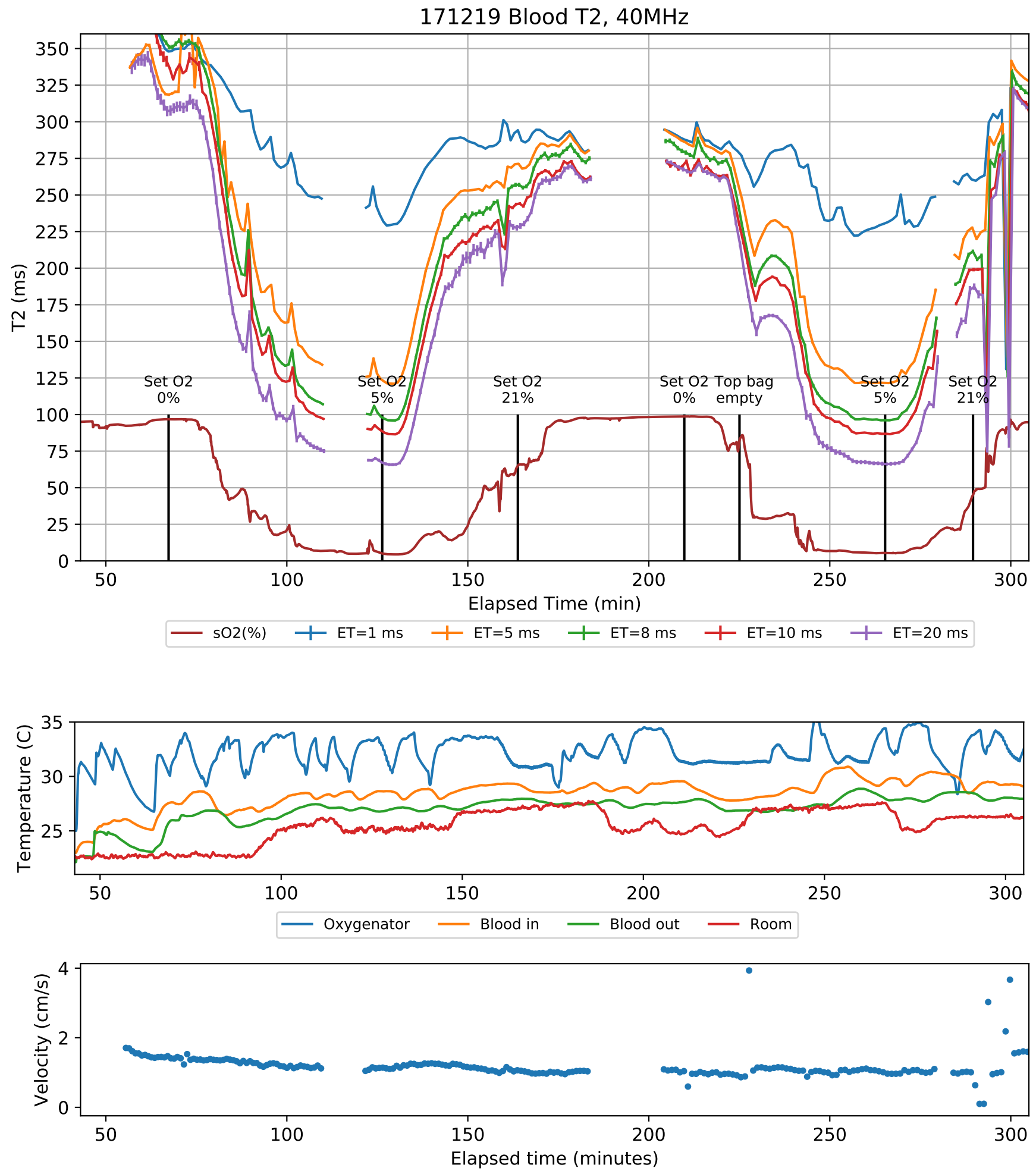

Figure 5.7: $T_{2}$ measurements during blood oxygenation ramping at $40 \mathrm{MHz}$ field. Error bars show $T_{2}$ fitting error. $H c t=0.35 . \mathrm{v}=(1.00 \pm 0.15) \mathrm{cm} / \mathrm{s}$ between $55-280$ minutes. Temperature increased from $26^{\circ} \mathrm{C}$ to $29^{\circ} \mathrm{C}$ from $55-280$ minutes. 
The blood was then reoxygenated in two stages. The NMR experiment was switched off around minute 280, and there appear to be artefacts at minute 293 and 300. At the third oxygenated stage, the $T_{2}$ reached $320 \mathrm{~ms}$, which is still lower than the initial value, but is higher than the second oxygenated level.

Because of the artefacts in ramp up 1, ramp down 2 and ramp up 2, only the first deoxygenation ramp has been used for further analysis.

\subsection{2 $20 \mathrm{MHz}$}

Immediately after the experiment at a field of $40 \mathrm{MHz}$, the current in the magnet was dropped to produce a $20 \mathrm{MHz}$ field. The probe was re-tuned and matched for the lower frequency, and pulse power calibration was checked (although the same coil was used, there may have been changes in the tuning and matching at a different frequency). The results are shown in Figure 5.8.

In the $20 \mathrm{MHz}$ experiment, the initial $T_{2}$ is around $275 \mathrm{~ms}$ for all echo times, which is lower than in the $40 \mathrm{MHz}$ experiment. While this is likely caused by the same process lowering $T_{2}$ in the $40 \mathrm{MHz}$ experiment, there may also be a contribution from the increased flow rate in this experiment. There is still a significant drop as the blood is deoxygenated, and the splitting between different echo times is still clear. For the deoxygenated blood, the $T_{2}$ at the $1 \mathrm{~ms}$ echo time is $237 \mathrm{~ms}$, while the $20 \mathrm{~ms}$ echo time gives a $T_{2}$ of $135 \mathrm{~ms}$. As expected, the size of the change from oxygenated to deoxygenated is smaller than in the $40 \mathrm{MHz}$ experiment.

When the blood is reoxygenated, the $T_{2}$ increases to $250 \mathrm{~ms}$ for all echo times. As in the $40 \mathrm{MHz}$ experiment, the looping batch experiments was interrupted between minutes 475-485 and 522-534 to do CPMGvT experiments.

The same pattern is seen in the second deoxygenation-oxygenation ramp. At minute 520 , there is a large spike in $T_{2}$, this is due to plasma and red blood cells separating in the blood. When the blood is no longer homogeneous, the plasma creates a second relaxation component with a much longer $T_{2}(>500 \mathrm{~ms}$ for separated plasma) that increases the measured $T_{2}$. This was fixed by squeezing the tubes slightly to mix the blood. There were also issues caused by the blood in the blood bags separating into regions with different oxygenation. When these were pulled into the outlet of the bag, they created slugs of blood in the tube with a different oxygenation. To try and avoid this, the bags were mixed by gentle shaking.

As both of the deoxygenation ramps were smooth, they were both extracted for further analysis. 
171219 Blood T2, 20MHz
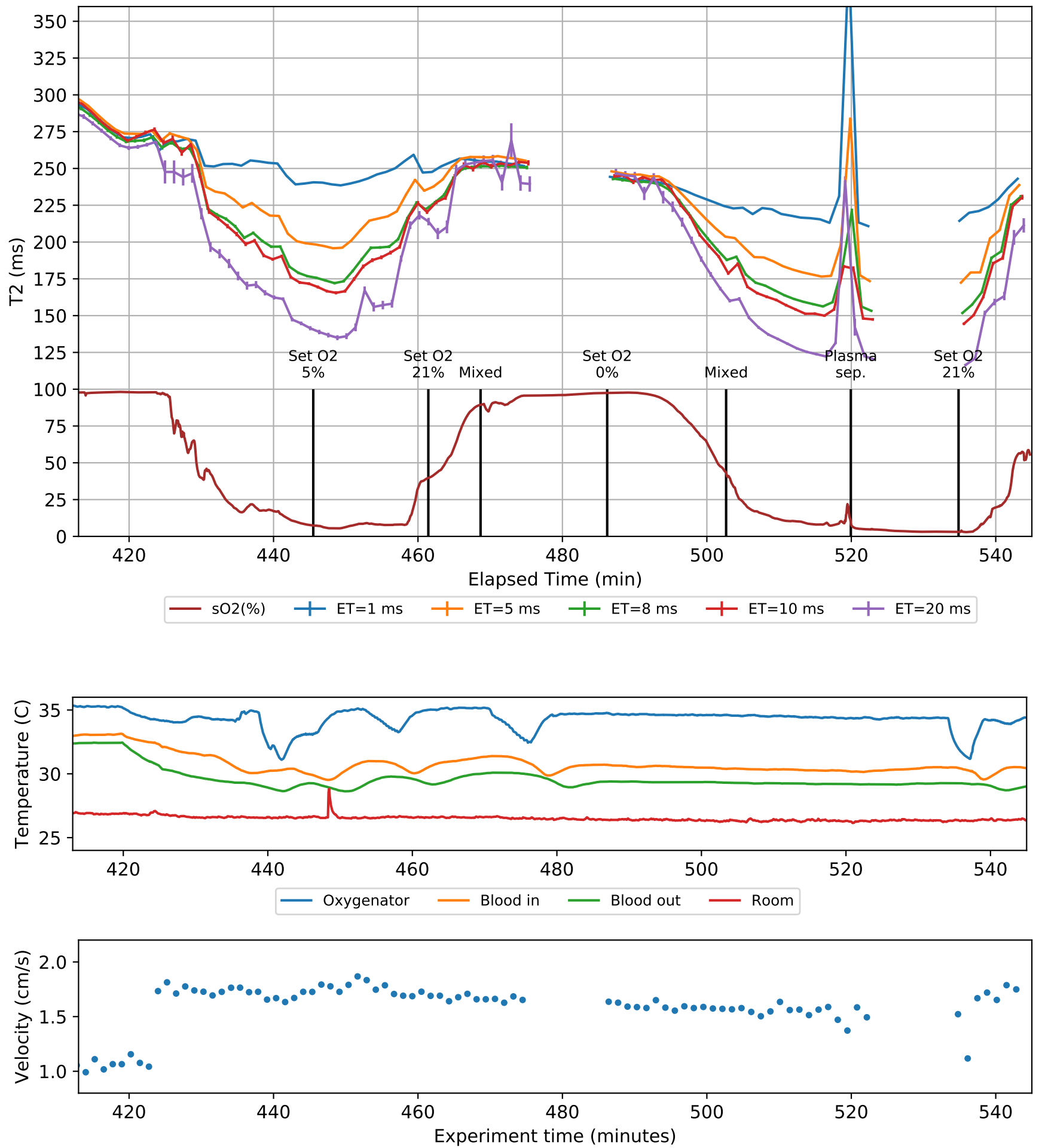

Figure 5.8: $T_{2}$ measurements during blood oxygenation ramping at $20 \mathrm{MHz}$ field. Error bars show $T_{2}$ fitting error. $H c t=0.35 . \mathrm{v}=(1.9 \pm 0.1) \mathrm{cm} / \mathrm{s}$ between $425-515$ minutes. Temperature decreased from $33^{\circ} \mathrm{C}$ to $29^{\circ} \mathrm{C}$ from $425-515$ minutes. 


\subsection{3 $14 \mathrm{MHz}$}

The blood sample was collected 11 days before the experiment, and the results are shown in Figure 5.9. The $\mathrm{pH}$ was 7.14 after bringing up to temperature, and haematocrit was 0.33 . In the $14 \mathrm{MHz}$ field experiment, the initial $T_{2}$ is approximately $315 \mathrm{~ms}$ for all echo times. As the $\mathrm{sO}_{2}$ is decreased, $\mathrm{T}_{2}$ decreases, and the splitting between echo times becomes visible. When the blood is deoxygenated, the $T_{2}$ at the $1 \mathrm{~ms}$ echo time is $283 \mathrm{~ms}$, and $206 \mathrm{~ms}$ for the $20 \mathrm{~ms}$ echo time. Both the size of the decrease from oxygenated to deoxygenated, and the $80 \mathrm{~ms}$ splitting between the echo times is smaller than found in the $20 \mathrm{MHz}$ experiments, as expected from theory. When compared with the stopped flow experiments at a field $12 \mathrm{MHz}$ the difference between oxygenated and deoxygenated is roughly the same, which suggests that the flow is not having a large effect.

Reoxygenating occured using only one step with $21 \% \mathrm{O}_{2}$. This caused a relatively fast increase in the $\mathrm{sO}_{2}$, particularly at low $\mathrm{sO}_{2}$ values where the $\mathrm{T}_{2}$ change is most rapid. This limited the number of points that could be collected during this part oxygenation ramp, e.g. around minute 110 .

There is only a slight drop in the $T_{2}$ at the second oxygenated stage when compared to the initial value ( $315 \mathrm{~ms}$ to $300 \mathrm{~ms}$ ). However, the second deoxygenated stage has a slightly smaller splitting between the echo times, although this may be because the first deoxygenation stage reached a lower $\mathrm{sO}_{2}$.

At minute 141, the optical sensor shows a slug of unmixed deoxygenated blood from the upper blood bag going past. This is also shown in the NMR data, which shows the $T_{2}$ from short and long echo times split by $20 \mathrm{~ms}$. The splitting here is smaller when compared to the splitting at the same $\mathrm{sO}_{2}$ from the first deoxygenation section, which is $50 \mathrm{~ms}$. This suggests that the saturation has increased by the time it has reached the NMR probe.

A similar effect can be seen at minute 218, where the splitting between short and long echo times drops to under $5 \mathrm{~ms}$. This suggests a slug of oxygenated blood has gone through the probe. Interestingly this is not really shown on the optical sensor, which only shows a small spike.

Because of the rapid increases in $T_{2}$ during the oxygenation ramps, and the $T_{2}$ spike in the second deoxygenation ramp, only the data from the first deoxygenation ramp is used for further analysis. 

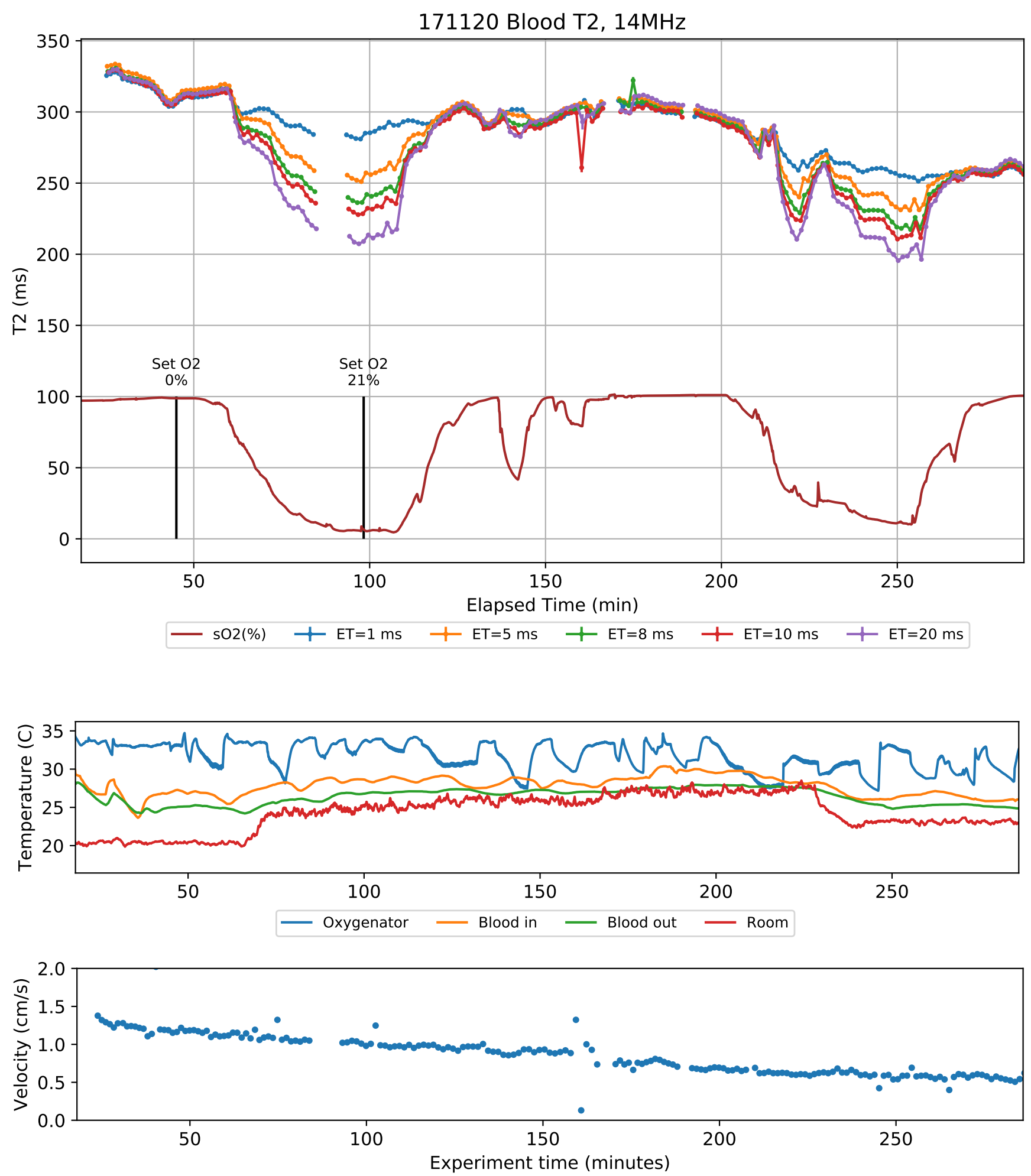

Figure 5.9: $T_{2}$ measurements during blood oxygenation ramping at $14 \mathrm{MHz}$ field. Error bars show $T_{2}$ fitting error. $H c t=0.33 . \mathrm{v}=1.2 \mathrm{~cm} / \mathrm{s}$ to $0.7 \mathrm{~cm} / \mathrm{s}$ between $20-286$ minutes. Temperature varied from $25^{\circ} \mathrm{C}$ to $29^{\circ} \mathrm{C}$ during experiment. Last two gas mix change times not recorded. 


\subsection{4 $10 \mathrm{MHz}$}

The blood sample used in the $10 \mathrm{MHz}$ experiment was collected 13 days beforehand. After warming up, the $\mathrm{pH}$ was 7.19 and haematocrit was 0.34 . The results are shown in Figure 5.10.

The initial $T_{2}$ in this experiment is around $3250 \mathrm{~ms}$. At this oxygenation stage, there is a $20 \mathrm{~ms}$ split in the $T_{2}$ between short and long echo times. In other experiments, this splitting was negligible, so this effect may be due to a change in the experimental setup at this field e.g. the magnet shimming, or a higher flow rate setting.

As the $\mathrm{sO}_{2}$ is decreased, the $T_{2}$ falls from $325 \mathrm{~ms}$ to $300 \mathrm{~ms}$ at the $1 \mathrm{~ms}$ echo time, and from $300 \mathrm{~ms}$ to $250 \mathrm{~ms}$ at the $20 \mathrm{~ms}$ echo time. The split increases to approximately $50 \mathrm{~ms}$ when deoxygenated, which is smaller than the $80 \mathrm{~ms}$ found at $14 \mathrm{MHz}$. In the second oxygenated stage, the $T_{2}$ at the $1 \mathrm{~ms}$ echo time is slightly lower than the initial value. Additionally, the same splitting occurs between the short and long echo times as the first oxygenated stage.

Interestingly, this shows oscillations in the $T_{2}$ value between minute 120-160 . These are well correlated with an oscillation in the blood temperature, which varied between $27^{\circ} \mathrm{C}$ to $28^{\circ} \mathrm{C}$. This oscillation was not observed in the other samples, and the size of the temperature dependence is larger than would be expected from other experiments (see $5 \mathrm{MHz}$ data).

In the second deoxygenated stage, the $T_{2}$ values are $20 \mathrm{~ms}$ lower than the first deoxygenated stage. The splitting between short and long echo times remains $50 \mathrm{~ms}$. After reoxygenation, the third oxygenated stage is lower again by $23 \mathrm{~ms}$. These are the same sort of decreases observed in the higher field experiments.

Both deoxygenation ramps produced the expected smooth decrease in $T_{2}$, so both were used for further analysis.

\subsection{5 $5 \mathrm{MHz}$}

The blood sample used in the $5 \mathrm{MHz}$ experiment was collected the day before. After being warmed up, the $\mathrm{pH}$ was 7.20, and the haematocrit was 0.34 . Results at the $5 \mathrm{MHz}$ field are shown in Figure 5.11.

The initial $T_{2}$ for this sample is at $286 \mathrm{~ms}$ for the $1 \mathrm{~ms}$ echo time. Like the 10 $\mathrm{MHz}$ experiment, the $T_{2}$ values for short and long echo times show a $20 \mathrm{~ms}$ split.

When the $\mathrm{sO}_{2}$ is dropped, the $\mathrm{T}_{2}$ also decreases from $300 \mathrm{~ms}$ to $286 \mathrm{~ms}$ for the $1 \mathrm{~ms}$ echo time, and from $266 \mathrm{~ms}$ to $232 \mathrm{~ms}$ for the $20 \mathrm{~ms}$ echo time. The $20 \mathrm{~ms}$ 
171122 Blood T2, 10MHz
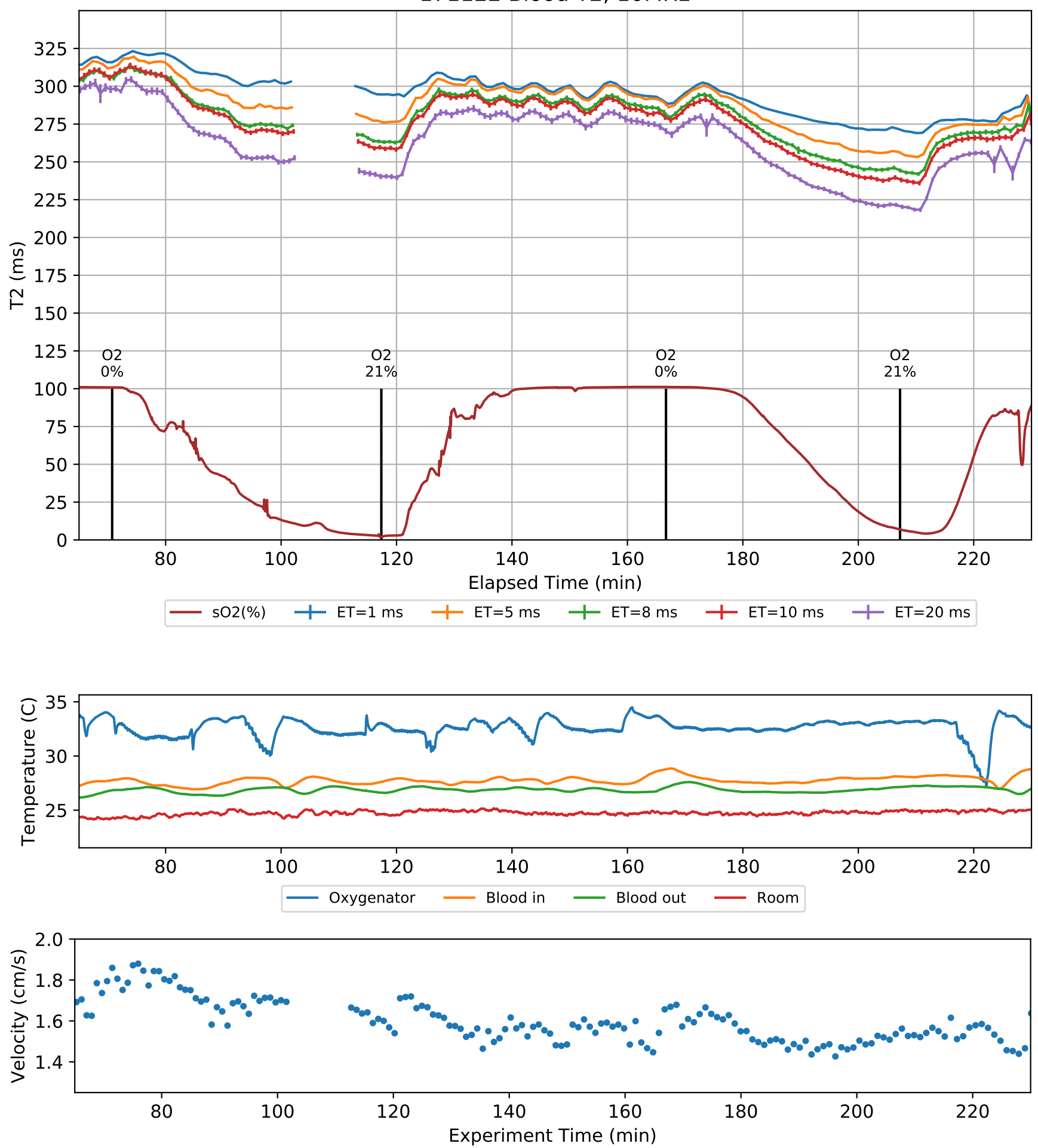

Figure 5.10: $T_{2}$ measurements during blood oxygenation ramping at $10 \mathrm{MHz}$ field. Error bars show $T_{2}$ fitting error. $H c t=0.34 . \mathrm{v}=(1.6 \pm 0.1) \mathrm{cm} / \mathrm{s}$ between $70-230$ minutes. Temperature was stable at $(28.0 \pm 0.5){ }^{\circ} \mathrm{C}$ during experiment. 
drop at short echo time is roughly the same as the stopped flow experiment, which decreased by $25 \mathrm{~ms}$ when samples were deoxygenated.

The splitting between short and long echo times is only $30 \mathrm{~ms}$ which is quite close to the splitting in the first oxygenated stage (at 90 minutes). During reoxygenation, the small size of the $T_{2}$ change means that it does not appear to come back up, as the background $T_{2}$ decrease is of the same size at this point. However the splitting between the $1 \mathrm{~ms}$ and $20 \mathrm{~ms}$ echo times returns to $20 \mathrm{~ms}$. In the second oxygenated and deoxygenated stages, the same change in splitting occurs, even though the absolute $T_{2}$ value is still decreasing.

After noticing the relatively high flow rate, the flow rate was lowered from around $2.3 \mathrm{~cm} / \mathrm{s}$ to $1.4 \mathrm{~cm} / \mathrm{s}$ at 265 minutes. The $T_{2}$ increases to $290 \mathrm{~ms}$, which is expected as high flow rates decrease the observed $T_{2}$. Interestingly, this also caused the splitting between the short and long echo times to decrease to around $10 \mathrm{~ms}$.

Dips at minutes 273 and 286 occur at the same time the temperature dipped $4{ }^{\circ} \mathrm{C}$, due to changing the settings on the flow rate through the oxygenator. When the blood is deoxygenated to $\mathrm{sO}_{2}=17 \%$, the $T_{2}$ for the $1 \mathrm{~ms}$ echo time decreases from $260 \mathrm{~ms}$ to $245 \mathrm{~ms}$. The splitting between short and long echo times increases to $20 \mathrm{~ms}$ at the maximum (around minute 350 ). This $T_{2}$ change due to oxygenation is approximately the same size as the change observed at the faster flow rate.

When reoxygenated, the $T_{2}$ increases back to $255 \mathrm{~ms}$ at the $1 \mathrm{~ms}$ echo time, and $245 \mathrm{~ms}$ at the $20 \mathrm{~ms}$ echo time. The splitting of $10 \mathrm{~ms}$ is consistent with the value when it was oxygenated.

Because of the change in flow rate, only the data from the second deoxygenation ramp was extracted for further analysis.

\subsubsection{Dependence on $\mathrm{sO}_{2}$}

To compare the change in $T_{2}$ from experiments with different field strengths, data points from the smooth, monotonic parts of the $T_{2}$ curves were extracted and matched with the $\mathrm{sO}_{2}$ measurements from the optical sensor. This matching process took into account the delay between the optical sensor and the NMR probe. Where data was taken from multiple oxygenation ramps, the $T_{2}$ values were shifted up, so that $T_{2}$ of oxygenated blood matched the initial measurements. This removes the effect of the background changes in $T_{2}$ (effectively $T_{20}$ in the Luz-Meiboom equation). These results are shown in Figure 5.12.

These plots clearly show the increases in $R_{2}$ (decrease in $T_{2}$ ) as $s \mathrm{O}_{2}$ decreases. 
171129 Blood T2, 5MHz
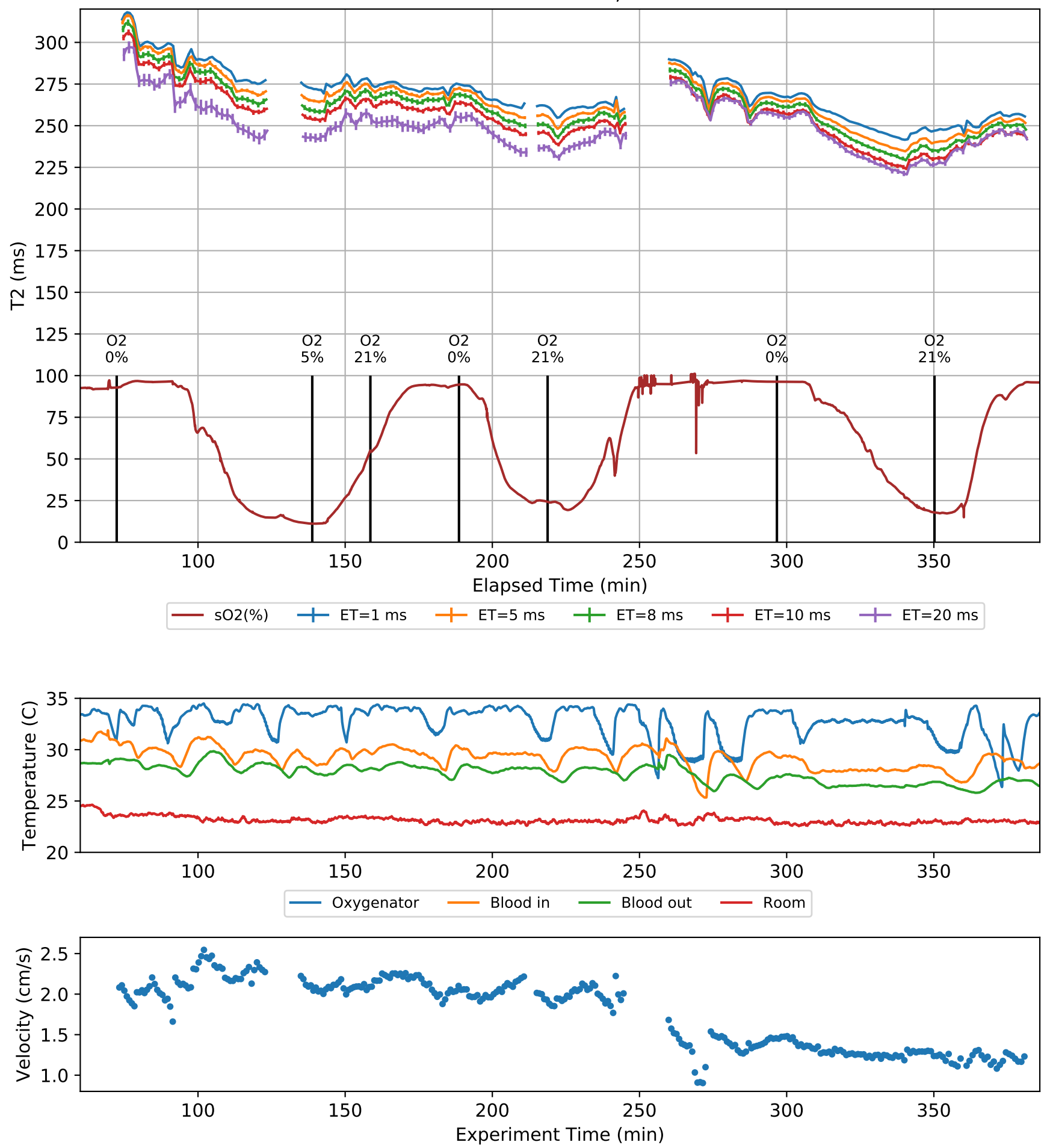

Figure 5.11: $T_{2}$ measurements during blood oxygenation ramping at $5 \mathrm{MHz}$ field. Error bars show $T_{2}$ fitting error. $H c t=0.35 . \quad \mathrm{v}=(2.3 \pm 0.4) \mathrm{cm} / \mathrm{s}$ between $80-250$ minutes, and varied from $(1.4 \pm 0.3) \mathrm{cm} / \mathrm{s}$ between $280-380$ minutes. Temperature varied around $(29 \pm 3)^{\circ} \mathrm{C}$ during experiment, with a drop to $25^{\circ} \mathrm{C}$ at 273 minutes. 
The higher field strengths also have a much wider range in $R_{2}$, where the $40 \mathrm{MHz}$ experiment has $R_{2}$ values between $3.5 \mathrm{~s}^{-1}$ and $13.5 \mathrm{~s}^{-1}$, and the $10 \mathrm{MHz}(\mathrm{d})$ experiment has values between $3.2 \mathrm{~s}^{-1}$ and $4 \mathrm{~s}^{-1}$ (the $5 \mathrm{MHz}$ experiment (e) does not reach the lower levels of $s \mathrm{O}_{2}$, so has a smaller range.) The slope of the $\mathrm{R}_{2}$ curves also increases at lower values of $\mathrm{sO}_{2}$, which agrees with theory, which predicts a dependence on $\left(1-\mathrm{sO}_{2}\right)^{2}$. This effect is more visible at higher fields, and in the 10 $\mathrm{MHz}(\mathrm{d})$ and $5 \mathrm{MHz}$ (e) experiments, the data appears almost linear.

$R_{2}$ measurements with longer echo times are also much more sensitive to the decreasing $\mathrm{sO}_{2}$. This is because there is more time for protons to experience dephasing from the field inhomogeneity as the time between the refocusing pulses increases. These changes were not as visible in the stopped flow experiments due to the inhomogeneity of the magnets, which caused additional dephasing.

The splitting between long and short echo times also increases as $\mathrm{sO}_{2}$ decreases, as the induced field inhomogeneity is dependent on the fraction of deoxy-haemoglobin in red blood cells, which is $\left(1-\mathrm{sO}_{2}\right)$. There is also a stronger effect at higher fields, as the induced inhomogeneity becomes stronger. However, at the $5 \mathrm{MHz}$ field (e), changes in $R_{2}$ across echo time are small enough to be within the uncertainty from fitting.

\subsubsection{Dependence on Field}

Plotting the difference in $R_{2}$ between deoxygenated and oxygenated blood shows the same trends. Figure 5.13 shows that $R_{2}$ change increases with field strength, going from $(0.35 \pm 0.30) \mathrm{s}^{-1}$ at $0.1 \mathrm{~T}$ to $(1.14 \pm 0.06) \mathrm{s}^{-1}$ at $1 \mathrm{~T}$ for the $1 \mathrm{~ms}$ echo time. As expected, the $5 \mathrm{~ms}$ echo time shows a larger $R_{2}$ change, increasing to $(4.60 \pm 0.06) \mathrm{s}^{-1}$ at $1 \mathrm{~T}$. The trends of both echo times look like they may follow the quadratic trend predicted by theory, but more data points would be needed to confirm this.

While this falls within the uncertainty of the result from the stopped flow experiment on the MOLE, the Halbach measured a slightly larger $R_{2}$ change at both echo times. This may be due to effects from the background gradients not being completely removed when subtracting the relaxivities. Conversely, the Spinsolve measurement at $5 \mathrm{~ms}$ echo time is about half as large $\left(2.96 \mathrm{~s}^{-1}\right)$ than the measurement at $1 \mathrm{~T}$. The deoxygenated Spinsolve sample had $\mathrm{sO}_{2}=9 \%$, rather than the $4 \%$ of the continuous flow experiment. Taking the measured $\mathrm{R}_{2}$ when $\mathrm{sO}_{2}=9 \%$ from the continuous flow experiment makes the relaxivity difference $4.1 \mathrm{~s}^{-1}$, which is still larger than the Spinsolve result. 
(a) $40 \mathrm{MHz}: \mathrm{v}=1.0 \mathrm{~cm} / \mathrm{s}$

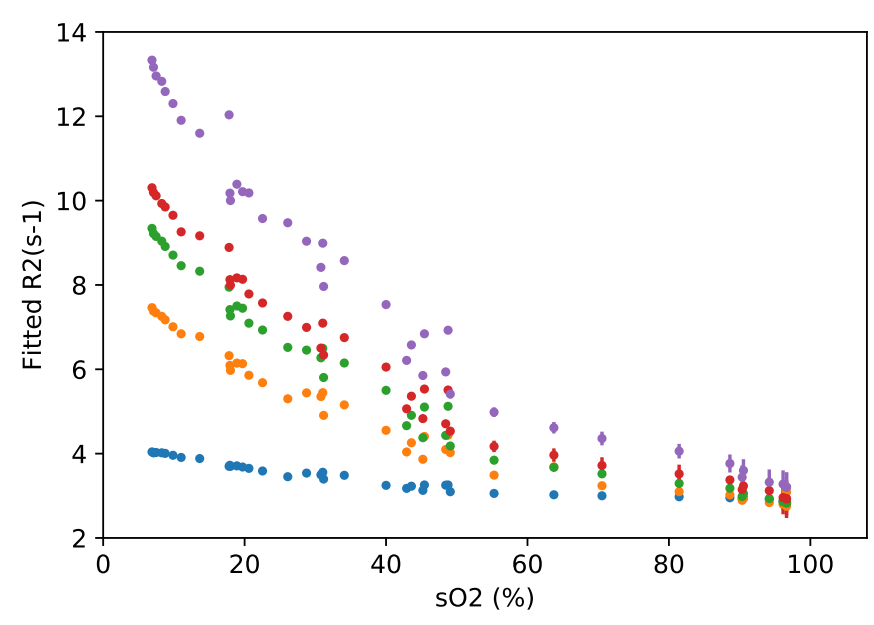

(c) $14 \mathrm{MHz}: \mathrm{v}=1.1 \mathrm{~cm} / \mathrm{s}$

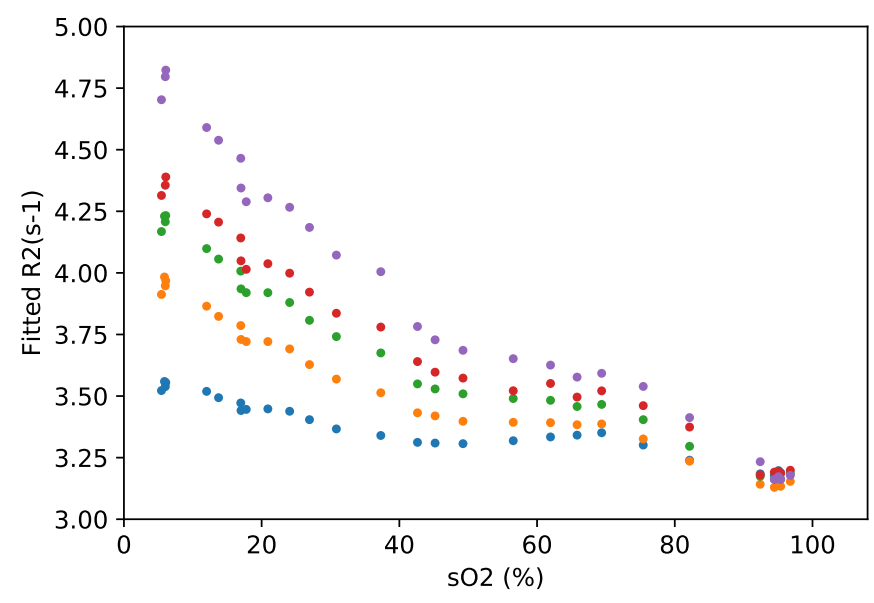

(e) $5 \mathrm{MHz}: \mathrm{v}=1.4 \mathrm{~cm} / \mathrm{s}$

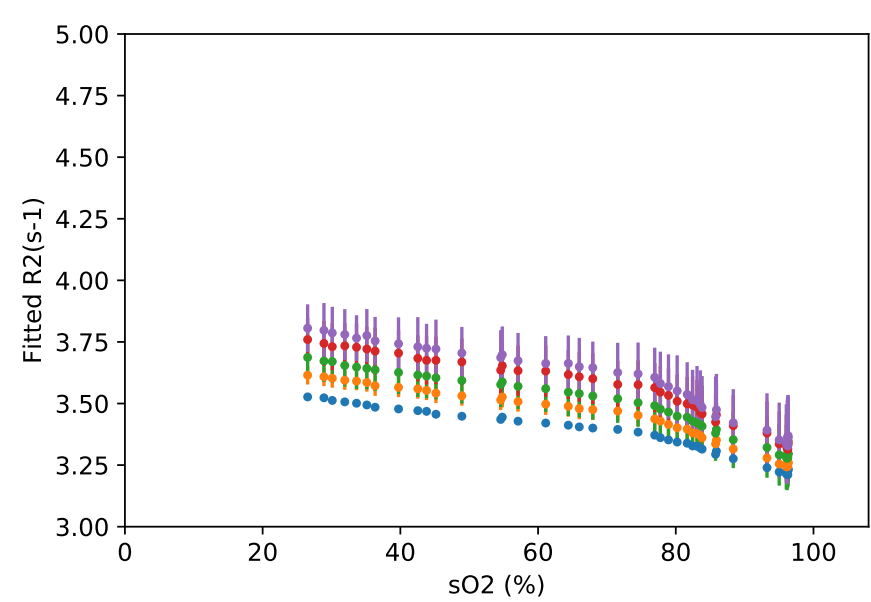

(b) $20 \mathrm{MHz}: \mathrm{v}=1.9 \mathrm{~cm} / \mathrm{s}$

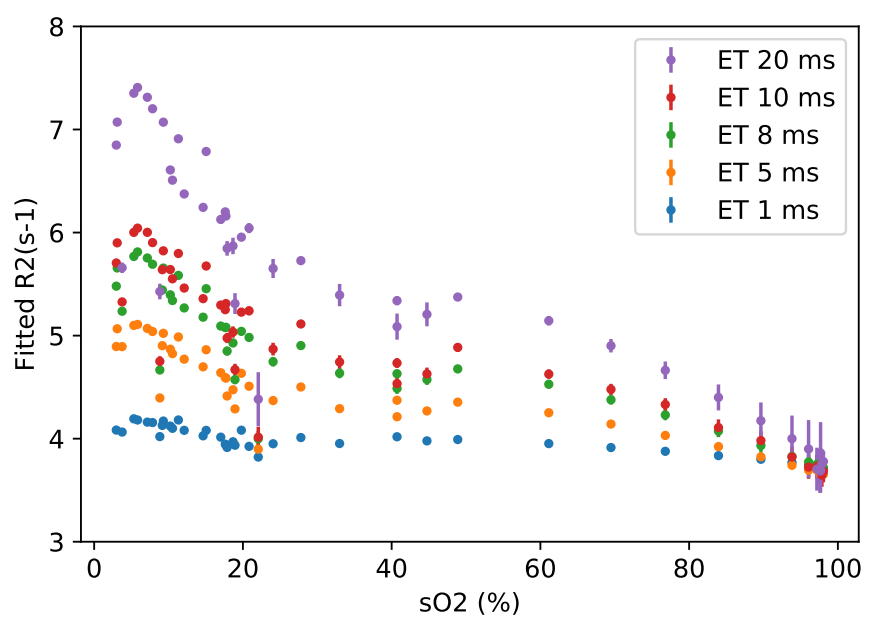

(d) $10 \mathrm{MHz}: \mathrm{v}=1.6 \mathrm{~cm} / \mathrm{s}$

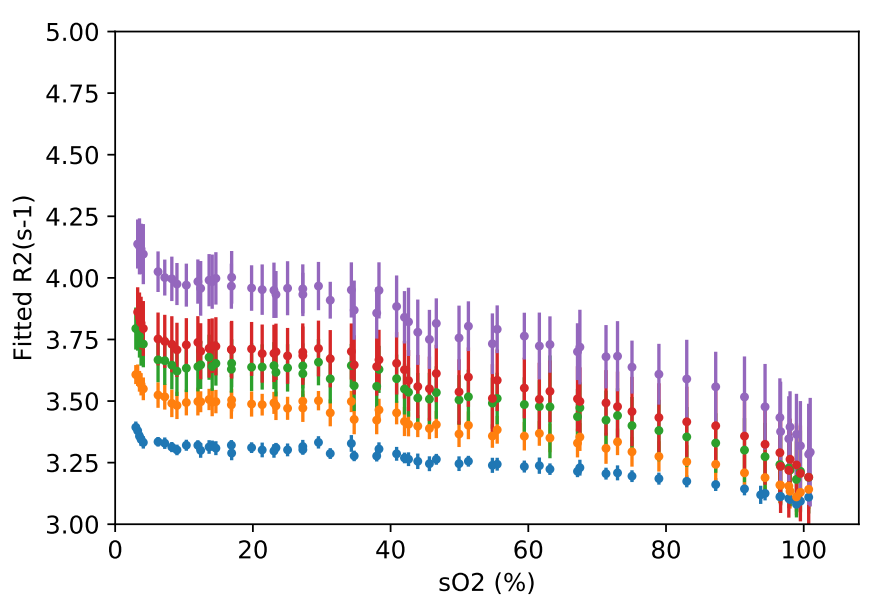

Figure 5.12: $\mathrm{R}_{2}$ during oxygenation ramps plotted against measured $\mathrm{sO}_{2}$, at different field strengths. Error bars show fitting error. Note different $R_{2}$ scales for 40 $\mathrm{MHz}$ and $20 \mathrm{MHz}$ data. The same symbols are used in all graph 


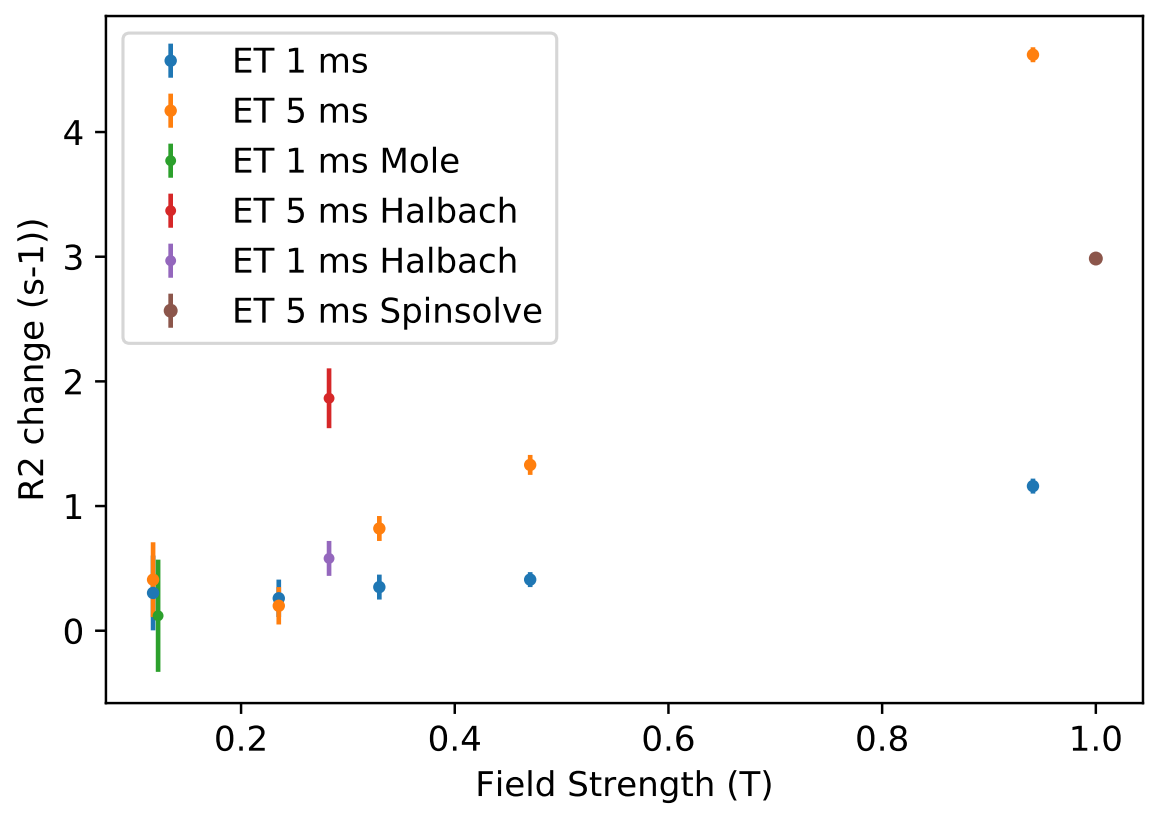

Figure 5.13: Change in $R_{2}$ at different fields for $1 \mathrm{~ms}$ and $5 \mathrm{~ms}$ echo times, also showing stopped flow results at these echo times. Data points show $R_{2}$ deoxy $-R_{2}$ oxy. Deoxygenated sample in $5 \mathrm{MHz}$ data had $\mathrm{sO}_{2}=8 \%$, all others had $\mathrm{sO}_{2}<5 \%$

To more easily show the dependence on field, the $T_{2}$ values from different echo times at each $\mathrm{sO}_{2}$ were fit to find $\mathrm{K}_{0}$ in the Luz-Meiboom equation Equation 2.14, following the same procedure as Stefanovic et al. [26]. This parameter describes the splitting between the echo times, and inspecting Equation 2.12 and Equation 2.14 shows it should be proportional to:

$$
\gamma^{2} K_{0}=(H c t)(1-H c t)\left(1-s O_{2}\right)^{2} \alpha^{2} \omega_{0}^{2}
$$

Each data point in the $\mathrm{R}_{2}$ vs $\mathrm{sO}_{2}$ curves was fitted using non-linear fitting to find $K_{0}$, using $\tau_{e x}=3 \mathrm{~ms}$ for the exchange time, and $T_{20}$ as a second fitted variable. This value of $\tau_{e x}$ was reported by Stefanovic [26], and $T_{20}$ was allowed to vary to ensure that the $K_{0}$ was only dependent on the difference between echo times (as it adds a uniform offset). Fitting the Luz-Meiboom equation to the different $R_{2}$ values at the 5 echo times gave good agreement to the data across all $\mathrm{sO}_{2}$ values. These results are included in Figure 5.14.

Plotting $\mathrm{K}_{0}$ against $\left(1-\mathrm{sO}_{2}\right)^{2}$ shows a linear relationship at all field strengths, as predicted by theory. These were fit using weighted least-squares fitting to find the slope. Fit parameters for the linear fits are shown in Table 5.2. At high values of $\mathrm{sO}_{2}$ (left side of graph), the fitted $K_{0}$ parameters should decrease to 0 , as the dependence on echo time and therefore the splitting between $T_{2}$ values should go 


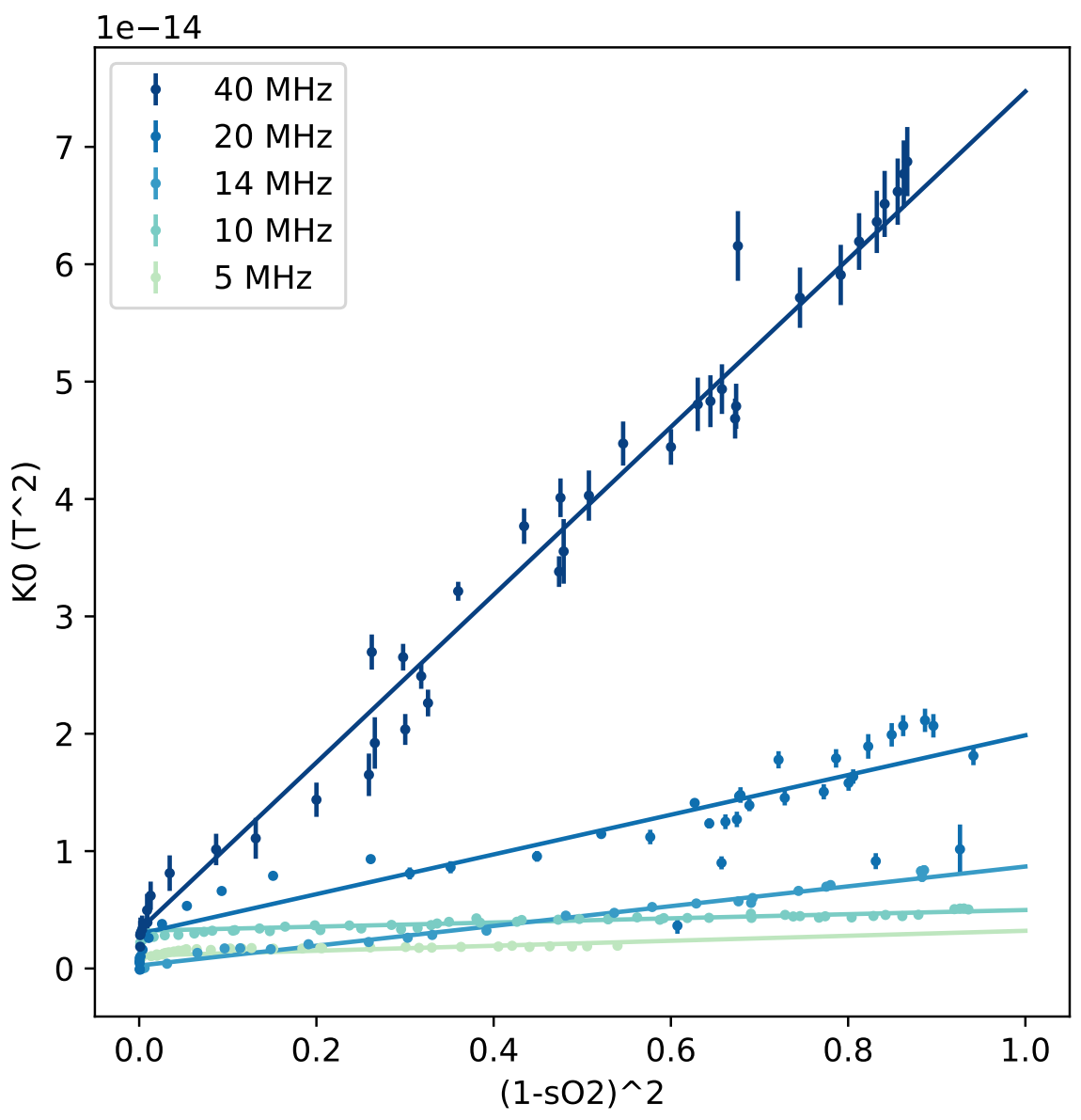

Figure 5.14: Dependence of $K_{0}$ on $s O_{2}$ at different fields. $K_{0}$ values found by fitting as described in text. Linear fit coefficients are included in Table 5.2. Note $5 \mathrm{MHz}$ data only extends to $\mathrm{sO}_{2}=25 \%$.

to zero. In cases where oxygenated blood samples created splitting, for example the $10 \mathrm{MHz}$ data, this is reflected in the intercept parameter being non-zero.

As expected, the slope also increases with field strength. To determine the dependence on $B_{0}$, the slopes can be plotted on a log-log scale to see if they follow a linear trend. This is shown in Figure 5.15. The line of best fit has a slope of $2.1 \pm 0.4$, which is consistent with a dependence on $B_{0}{ }^{2}$.

\subsection{Discussion}

These continuous flow experiments show that decreases in blood $\mathrm{sO}_{2}$ cause decreases in $T_{2}$, and that these changes are still visible at lower field strengths. The $T_{2}$ decrease is larger when measured with longer echo times, and at higher fields, 


\begin{tabular}{|c|cc|c|}
\hline Field $(\mathrm{MHz})$ & Slope $\left(10^{-14} \mathrm{~T}^{2}\right)$ & Intercept $\left(10^{-14} \mathrm{~T}^{2}\right)$ & $r^{2}$ \\
\hline 5 & $0.21 \pm 0.02$ & $0.11 \pm 0.00$ & 0.91 \\
10 & $0.18 \pm 0.01$ & $0.32 \pm 0.00$ & 0.60 \\
14 & $0.84 \pm 0.03$ & $0.02 \pm 0.01$ & 0.95 \\
20 & $1.69 \pm 0.13$ & $0.30 \pm 0.05$ & 0.79 \\
40 & $7.15 \pm 0.18$ & $0.33 \pm 0.07$ & 0.94 \\
\hline
\end{tabular}

Table 5.2: Fit parameters showing dependence of $\mathrm{K}_{0}$ on $\mathrm{sO}_{2}$. Plotted in Figure 5.14

which agrees with theory.

The Luz-Meiboom equation (Equation 2.12) describes the change in $T_{2}$ as a function of $\mathrm{sO}_{2}, \mathrm{~B}_{0}\left(\omega_{0}\right), \mathrm{CPMG}$ echo time, and haematocrit (not tested in these experiments). In particular, it predicts that $R_{2} \propto\left[\omega_{0} \alpha\left(1-s O_{2}\right)\right]^{2}$. Figure 5.12 shows the dependence of $R_{2}$ in $s \mathrm{O}_{2}$, which at the higher fields (40-14 MHz) appears to follow the dependence on $\left(1-\mathrm{sO}_{2}\right)^{2}$. Removing the effect of echo time, by fitting $\mathrm{K}_{0}$ to the different experiments makes it easier to see this dependence in Figure 5.14. The fit results show that there is a significant linear correlation in the parameters at most of the different field strengths.

Additionally, fitting a log-log plot of the slopes of $K_{0}$ against the field strength $B_{0}$ (Figure 5.15) gives a slope of $2.1 \pm 0.4$. This agrees with the theoretical dependence on $B_{0}$ squared. The relationship seems to hold for all but the lowest field strength $(5 \mathrm{MHz})$, where the slope does not change significantly when compared to the $10 \mathrm{MHz}$ slope. This suggests that the splitting in $\mathrm{T}_{2}$ due to $\mathrm{sO}_{2}$ at the $5 \mathrm{MHz}$ field may be too small to be picked up with this set of 5 echo times. The dependence of echo time on $T_{2}$ has been investigated in more detail in chapter 6 , using a larger number of echo times.

\subsubsection{Confounding factors}

Other factors may also influence the $T_{2}$ measured in these experiments, and may add more uncertainty to the results. This includes physical parameters such as temperature and velocity. The temperature of the blood in the circuit was controlled using flow from a $37^{\circ} \mathrm{C}$ water bath through the oxygenator, although because of variations in the blood flow rate through the oxygenator, the mixing process which occured in the blood bags, and changes in the room temperature, in most cases the temperature was only stable within a $3-4^{\circ} \mathrm{C}$ range. In some experiments (e.g. the $10 \mathrm{MHz}$ experiment), a stable steady-state temperature was 


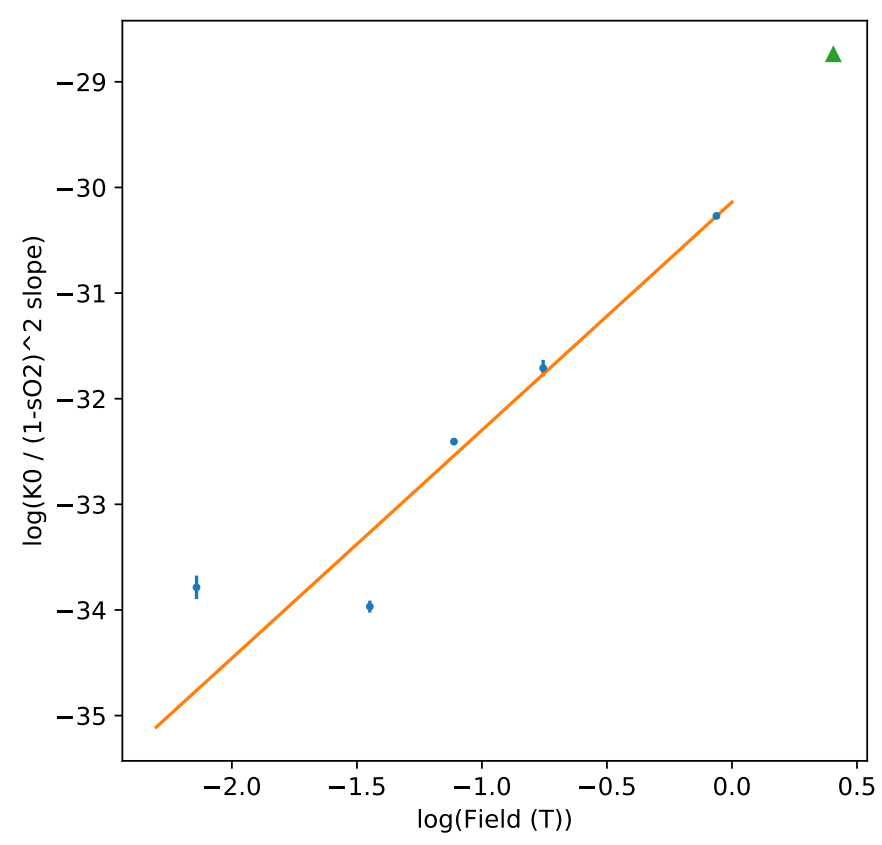

Figure 5.15: Log-log plot of $K_{0}$ slope against field strength. Green point is included from Stefanovic et al. for comparison [26]. Trend line has equation (2.1 \pm 0.4$)$ $\log \left(B_{0}\right)-(30.1 \pm 0.4)$

reached, which is the ideal case.

There are a number of examples in the experimental data where features in the temperature data are correlated with changes in $T_{2}$, for example in the $5 \mathrm{MHz}$ experiment (Figure 5.11), where a $4{ }^{\circ} \mathrm{C}$ drop causes a $15 \mathrm{~ms}$ dip in the $T_{2}$ at minute 273. This temperature drop is also correlated with a drop in the velocity measured by PGSE, from $1.6 \mathrm{~cm} / \mathrm{s}$ to $1.0 \mathrm{~cm} / \mathrm{s}$ (although it quickly recovers when the temperature returns to $29^{\circ} \mathrm{C}$ ). Interestingly the value of $T_{2}$ changed due to the temperature, the splitting between the $1 \mathrm{~ms}$ and $20 \mathrm{~ms}$ echo times stays at $130 \mathrm{~ms}$ through the dip. From this observation, there may be up to a $15 \mathrm{~ms}$ uncertainty in in $T_{2}$ measurements across some of the experiments.

Flow velocity can also affect the $T_{2}$ measurements. The experiments with doped water showed that the effect should be limited to $10 \mathrm{~ms}$ to $15 \mathrm{~ms}$ at the flow rates used. This is typically smaller than the changes due to $\mathrm{sO}_{2}$. However, over the hours-long time frame of the experiments, the flow rate typically dropped, even without adjusting the screw clamp. This may be due to problems with the setup, such as thermal expansion of the clamp or tubing, or the narrow gap in the tube being plugged by clotted blood. It may also be due to changes in the blood that cause it to flow slower, such as a changing viscosity. Interestingly, a decreas- 
ing velocity would mean that the $T_{2}$ should increase over time however, which is opposite to the decreasing trend visible in the data.

Flow may also cause increased dispersion, which creates different $T_{2}$ changes at different echo times. This effect may have been observed in the doped water experiments, where the $20 \mathrm{~ms}$ echo time $T_{2}$ measurements experienced a slightly larger dependence on the flow rate. This would affect the measurements based on $K_{0}$, which relies on the splitting between different echo times. While this was not taken into account in the fitting model, the $K_{0}$ fitting showed good agreement with the measured $R_{2}$ values to within $0.3 \mathrm{~s}^{-1}$ at the high field measurements, and within $0.05 \mathrm{~s}^{-1}$ in the 14,10 and $5 \mathrm{MHz}$ experiments.

In blood, the haematocrit is also known to affect the $T_{2}$ changes. The LuzMeiboom equation shows that $K_{0}$ is proportional to $(H c t)(1-H c t)$, which has a maximum when $H c t=0.5$. This is related to the proportions of protons in the RBC compartment and in the plasma. For the samples in these continuous flow experiments, the haematocrit was typically between $0.3-0.4$, after dilution with the anticoagulant solution in the blood collection process. It was not taken into account when calculating the dependence of $\mathrm{R}_{2}$ and $\mathrm{K}_{0}$ on $\mathrm{sO}_{2}$, and so it will be a confounding factor in these cases. The range of Hct in the continuous flow measurements was 0.33 to 0.38 , which corresponds to a change from 0.22 to 0.23 in the haematocrit factor. This represents an uncertainty of $\approx 5 \%$, which is smaller than the slope uncertainties in Table 5.2 (although this applies to comparing the different field strengths). In other studies, the effect of haematocrit on $T_{2}$, was found to follow the predicted trend $[43,30,44]$

\subsubsection{Comparison with literature}

As mentioned in the introduction, while there have been a number of studies of $T_{2}$ dependence on $\mathrm{sO}_{2}$ in the literature, most have been done at fields $>1.5 \mathrm{~T}$, and look at either field dependence with deoxygenated and oxygenated blood, or use one field to investigate the dependence on $\mathrm{SO}_{2}$. This makes it difficult to compare these continuous flow results with examples from the literature.

One comparable study was done by Brooks et al., who measured the dependence of $T_{2}$ on field strength between $0.02 \mathrm{~T}$ and $1.5 \mathrm{~T}$ for samples of oxygenated and deoxygenated blood [19]. They used a fixed echo time of $4 \mathrm{~ms}$, which is relatively close to the $5 \mathrm{~ms}$ echo time series used in this experiment. Their samples were much smaller $(0.5 \mathrm{ml})$ and were measured at 13 different $B_{0}$ strengths. Brooks et al. found that the difference between deoxygenated and oxygenated 
blood is given by $R_{2}=a+b B_{0}^{2}$, where $a=4 \mathrm{~s}^{-1}$ and $b=7.2 \mathrm{~s}^{-1} / \mathrm{T}^{2}$. This suggests that there is still a significant difference between the deoxygenated and oxygenated blood at low field.

In contrast the continuous flow results show a similar trend, but with $a=(0.11 \pm 0.12) \mathrm{s}^{-1}$ and $b=(5.1 \pm 0.3) \mathrm{s}^{-1} / \mathrm{T}^{2}$. While the $b$ value is approximately the same, the value for $a$ from these results means that there is little to no change between oxygenated and deoxygenated blood at low fields.

The reason for this difference is unclear, but may be due to differences in their sample preparation. They deoxygenated the blood chemically, which can completely remove oxygen in the samples and drop the $\mathrm{sO}_{2}$ to zero. They also state that the deoxygenated blood samples were deoxygenated and used without further processing, but the oxygenated blood samples were centrifuged, washed in saline, and resuspended in thawed plasma.

Gomori et al. also found little to no change in $T_{2}$ for weak $B_{0}$ fields. They found a $10 \mathrm{~ms} T_{2}$ difference between oxygenated and deoxygenated blood at $0.19 \mathrm{~T}$ [18] - at a $2 \mathrm{~ms}$ echo time, the oxygenated and deoxygenated blood had $T_{2}$ values of $(235 \pm 40) \mathrm{ms}$ and $(224 \pm 25) \mathrm{ms}$ (estimated from graph). This corresponds to an $R_{2}$ change of $(0.2 \pm 1.1) \mathrm{s}^{-1}$, which agrees with our finding of little to no change in $R_{2}$ at low field.

The other component of this experiment that can be compared is the dependence on $\mathrm{sO}_{2}$. Stefanovic et al. measured the $T_{2}$ of blood at various levels of oxygenation with a range of CPMG echo times in a $1.5 \mathrm{~T}$ MRI scanner [26]. They performed the same $\mathrm{K}_{0}$ fitting process to calibrate $\mathrm{K}_{0}$ values against $\mathrm{sO}_{2}$, and plotted them against the same $\left(1-\mathrm{sO}_{2}\right)^{2}$. Their results also produced a linear dependence, with a slope of $(3.1 \pm 0.2) \times 10^{-13} \mathrm{~T}^{2}$. For comparison, this data point is also included in Figure 5.15, and appears to follow the dependence on $B_{0}{ }^{2}$ predicted by theory.

Other studies give empirical calibration constants for the shape of the $\mathrm{R}_{2} / \mathrm{sO}_{2}$ curve (curvature in Figure 5.12). The results from these studies are used to calibrate $T_{2}$ measurements to $\mathrm{sO}_{2}$ in imaging studies using the simplified equation below.

$$
\frac{1}{T_{2}}=\frac{1}{T_{20}}+K\left(\tau_{180}, \omega_{0}\right)\left(1-\frac{s O_{2}(\%)}{100}\right)
$$

This curvature constant will be dependent on parameters such as field strength, echo time and haematocrit. One example is from Wright, who found $K=13.4 \mathrm{~s}^{-1}$ at a field of $1.5 \mathrm{~T}$, and a $6 \mathrm{~ms}$ echo time [21]. This value was also found by Portnoy, 
who reported $K=13.4 \mathrm{~s}^{-} 1 \mathrm{in}$ blood at $1.5 \mathrm{~T}$ [44]. Silvennoinen found a higher value of $K=21 \mathrm{~s}^{-1}$ using a spin-echo experiment at $1.5 \mathrm{~T}$ [25].

Fitting a quadratic curve to the $40 \mathrm{MHz}$ experiment (Figure 5.12a) gives a value of $K=5.1 \mathrm{~s}^{-1}$ at the $5 \mathrm{~ms}$ echo time (the closest to the echo time used by Wright). Extrapolating this to a $1.5 \mathrm{~T}$ field by multiplying by $\left(\frac{1.5 \mathrm{~T}}{0.94 \mathrm{~T}}\right)^{2}$ gives a value of $13 \mathrm{~s}^{-1}$, which is quite close to the published value. This extrapolated value would be expected to be lower, as these continuous flow experiments used blood samples with a lower haematocrit than the literature studies, which used blood with a physiological Hct, and a slightly shorter echo time (5 ms), rather than the $6 \mathrm{~ms}$ in studies by Wright).

\subsubsection{Background $T_{2}$ decrease: Haemolysis}

The general decreasing trend in $T_{2}$ over the time of the experiments is still significant in these experiments. A similar effect was also observed in the stopped flow experiments, where $T_{2}$ values did not fully recover after the blood samples were reoxygenated. In these continuous flow experiments, this effect is more visible due to the longer time frames and continuous monitoring.

The stopped flow experiments suggested that the change in $T_{2}$ might be due to the breakdown of red blood cells in the blood. To test this more quantitatively, the haemoglobin concentration was measured for samples of plasma, separated from blood removed from the flow circuit. This 'Plasma free haemoglobin' is an indicator for haemolysis, the breakdown of red blood cells, as the cells release their haemoglobin into the surrounding plasma [45]. Additionally, the $T_{2}$ of the separated plasma was measured on the Spinsolve, to see if there was any correlation with the changing $T_{2}$ values from the continuous flow experiment. In studies at high fields, haemoglobin has been shown in the literature to act as a paramagnetic contrast agent [31].

Plasma free haemoglobin was measured using the Kahn method, which is described in a review of haemolysis testing methods by Fairbanks [46]. This uses $\mathrm{UV} /$ Vis spectroscopy of the haemoglobin absorption peak at $578 \mathrm{~nm}$ to measure the concentration. The Harboe method was also tested: this method measures the haemoglobin absorption peak at $415 \mathrm{~nm}$, but this method requires dilution of the plasma sample. Instead, the coefficients were multiplied to remove the assumed dilution, although this did not take into account the changes that occur in absorbance due to the precipitation/dissolution of proteins and lipids in the plasma. Absorption spectra were measured in plastic cuvettes with a $1 \mathrm{~cm}$ 


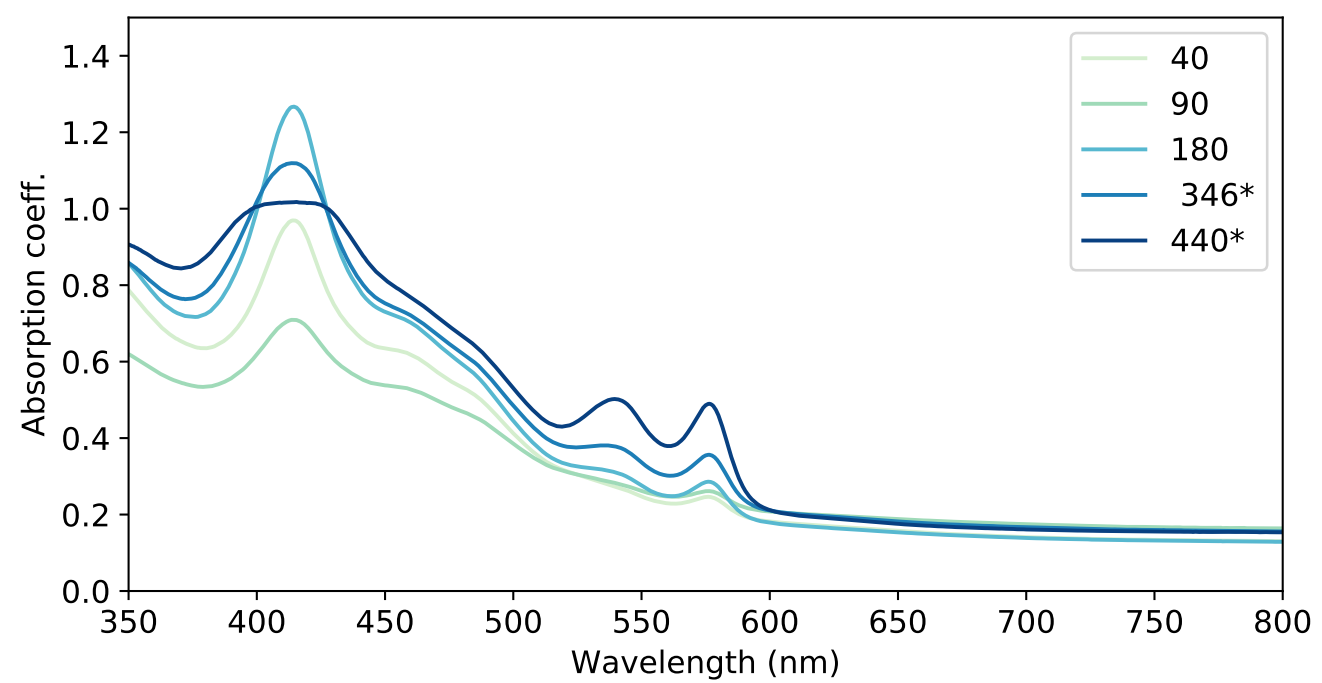

Figure 5.16: Absorption spectra for samples of separated plasma in $5 \mathrm{MHz}$ experiment. Different spectra sampled from circuit at time indicated. ${ }^{*}$ indicates sample was taken from deoxygenated blood.

pathlength, using a Shimadzu UV-2600 UV/Vis spectrometer (Shimadzu, Kyoto Japan).

$$
\begin{array}{cl}
\text { Kahn : } & c_{H b}(\mathrm{~g} / \mathrm{L})=1.55 A_{578}-0.861 A_{562}-0.689 A_{598} \\
\text { Harboe : } & c_{H b}(\mathrm{~g} / \mathrm{L})=0.836 \times\left(2 A_{415}-A_{380}-A_{450}\right) \times \frac{1}{11}
\end{array}
$$

These studies were done for the $40 \mathrm{MHz}, 10 \mathrm{MHz}$ and the $5 \mathrm{MHz}$ experiment. $\mathrm{UV} / \mathrm{Vis}$ spectra from the $5 \mathrm{MHz}$ experiment are shown in Figure 5.16. As the experiment time increases, the peaks at $415 \mathrm{~nm}$ and $570 \mathrm{~nm}$ grow, indicating an higher haemoglobin concentration. The $415 \mathrm{~nm}$ peak also appears to flatten out and decreases in height at longer times, this may be due to scattering, or interfering substances in the plasma samples. From these spectra, the plasma free haemoglobin at each point was calculated using equations 5.2 and 5.3, and these are shown in Figure 5.17.

The results from the $5 \mathrm{MHz}$ experiment show that there is a correlation between the increasing concentration of haemoglobin, and the $R_{2}$ of the separated plasma. As the concentration increases from $0.05 \mathrm{~g} / \mathrm{L}$ to $0.27 \mathrm{~g} / \mathrm{L}$, the $T_{2}$ values measured in plasma decrease from $757 \mathrm{~ms}$ to $667 \mathrm{~ms}$. As expected, the $T_{2}$ of the separated plasma is much longer than the blood measurements, around $700 \mathrm{~ms}$ vs $300 \mathrm{~ms}$, due to the removal of the red blood cells. This corresponds to an increase in $R_{2}$ from $1.3 \mathrm{~s}^{-1}$ to $1.5 \mathrm{~s}^{-1}$. Comparing this with the $R_{2}$ measurements in 
blood, this $0.2 \mathrm{~s}^{-1}$ change accounts for about half of the relaxivity change between minute 90 and minute 350, which is $0.4 \mathrm{~s}^{-1}$.

A similar result was found in the $40 \mathrm{MHz}$ experiment, although the plasma sample used was relatively cloudy, potentially due to lipids or proteins in the plasma causing the UV/Vis spectra to display broad absorptions. This interfered with the haemoglobin measurement, as it uses the region around the absorption peak to attempt to remove background absorption.

$R_{2}$ values in the separated plasma increased from $1.25 \mathrm{~s}^{-1}$ to $1.7 \mathrm{~s}^{-1}$, while the oxygenated blood increased from $2.9 \mathrm{~s}^{-1}$ to $4.0 \mathrm{~s}^{-1}$. While the UV/Vis spectra from this experiment cannot conclusively link free haemoglobin and the plasma $R_{2}$, the increasing trend in the plasma $R_{2}$ and blood $R_{2}$ over time suggest that the blood is breaking down.

These experiments suggest that the background decrease in $T_{2}$ across the time span of the experiments is due to haemolysis of the red blood cells. Haemolysis can be caused by many factors including the age of the blood, changes in osmolarity, temperatures above $40{ }^{\circ} \mathrm{C}$ and mechanical/shear stress [47]. This was observed happening in the pump when the occlusion was set too tightly, which lead to the use of the occlusion setting process described in subsection 3.1.1. It is possible that haemolysis is occurring due to the blood flowing through the relatively tight screw clamp, or due to contact with the PVC tube and oxygenator membrane. Blood haemolysis is known to occur in ex-vivo blood circulation circuits for cardiopulmonary bypass, although typically the natural scavenging process in the body is able to clear this without significant ill-effects [48].

\subsubsection{Oxygen saturation measurement from $T_{2}$}

As the goal of this project is to determine how well $\mathrm{sO}_{2}$ can be measured using NMR at low fields, the $T_{2}$ values measured in the $14 \mathrm{MHz}$ continuous flow experiment were used to back-predict the $\mathrm{s}_{2}$ for comparison to the optical sensor. In the literature, most experiments use a calibration curve to directly link measured $\mathrm{T}_{2}$ values with $\mathrm{s}_{2}$ for one echo time. This process was pioneered by Wright [21], and applied by Golay [43], Lu [34], Portnoy [49] and many others.

More recently, a parametric method of fitting $T_{2}$ values measured at multiple echo times was introduced by Varghese for use in MRI oximetry of the heart [35]. This relies on using non-linear least squares fitting to fit the full Luz-Meiboom equation (Equation 2.12, which includes terms for $\mathrm{Hct}, \alpha$ and $\mathrm{sO}_{2}$ ) to the measured $\mathrm{T}_{2}$ values and extracting $\mathrm{sO}_{2}$ from this. In their study, they demonstrated that 
(a) $5 \mathrm{MHz}$ experiment. ( $T_{2}$ for blood sample at minute 350 taken from minute 294 to compare data from oxygenated blood)
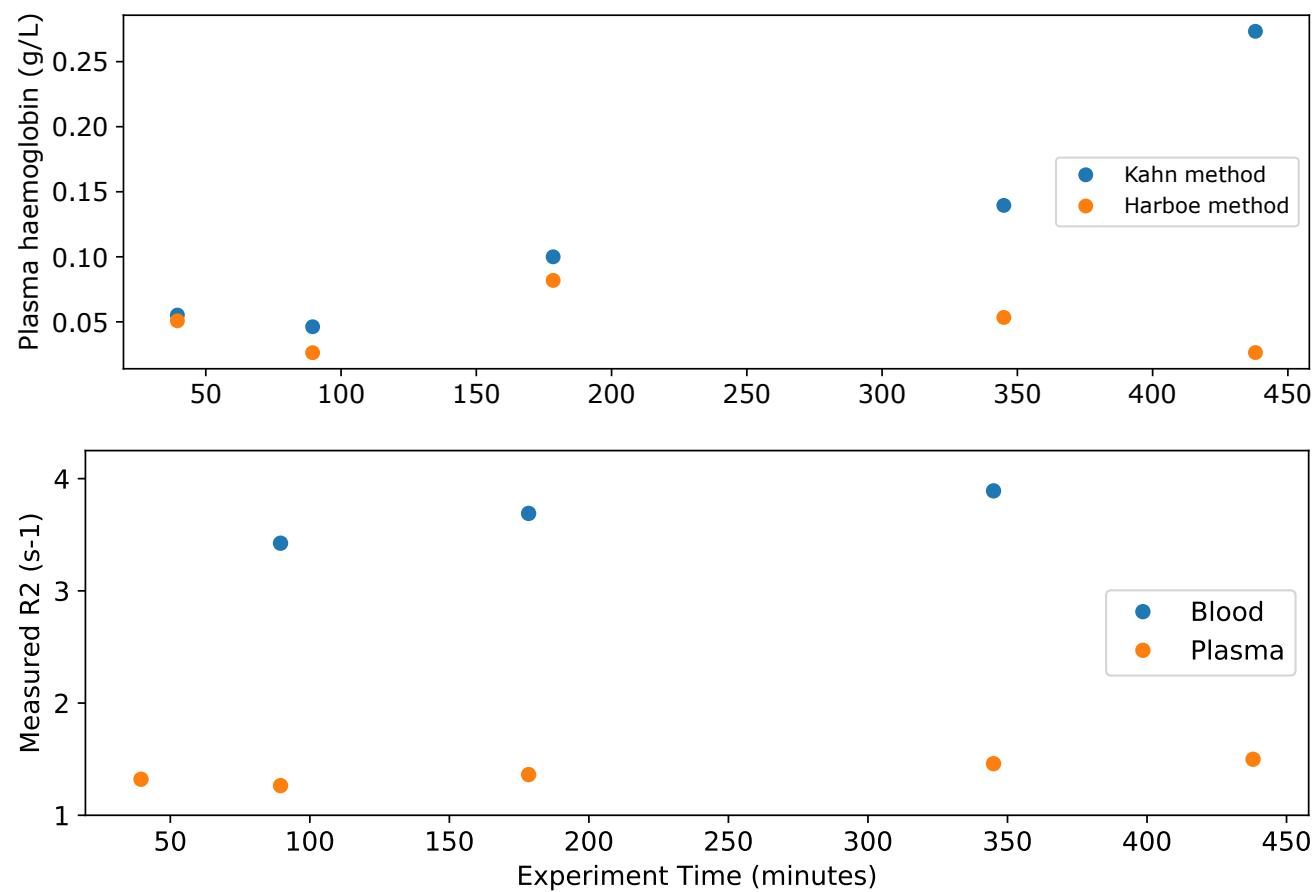

(b) $40 \mathrm{MHz} / 20 \mathrm{MHz}$ experiment
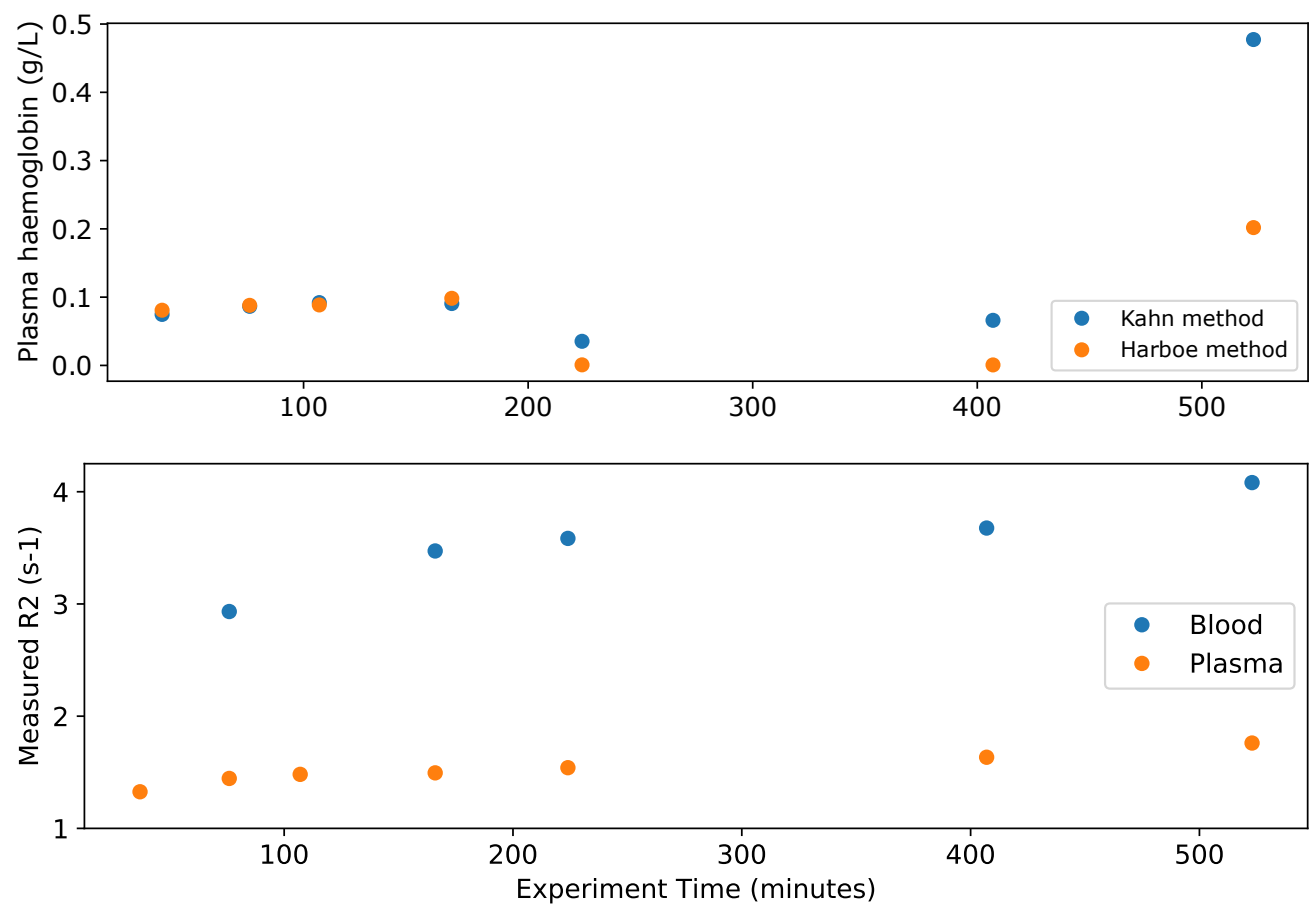

Figure 5.17: Blood haemolysis experiment result. Plasma free haemoglobin found using Kahn method, Plasma $R_{2}$ values measured on Spinsolve at $1 \mathrm{~T}$, Blood $R_{2}$ values taken from blood data shown in Figure 5.11 and Figure 5.7 
this parametric method improved $\mathrm{s}_{2}$ measurement accuracy when compared to the simplified calibration curve model, by comparing the $s \mathrm{O}_{2}$ values calculated with each method to direct $\mathrm{s}_{2}$ measurements of blood sampled from the heart (in pigs). This method requires a separate measurement of a sample of blood to find a known $\mathrm{sO}_{2}$ value and the haematocrit, to be able to estimate $\alpha$ and the haematocrit factor in the Luz-Meiboom equation. $T_{2}$ values were measured using 4 echo times between $10 \mathrm{~ms}$ to $25 \mathrm{~ms}$, which were used to estimate either the $\mathrm{sO}_{2}$, or 4 constrained parameters: $\mathrm{sO}_{2}, \mathrm{Hct}, \alpha$ and $\mathrm{T}_{20}$.

In this work, a similar parametric method was applied to find $K_{0}$ and $T_{20}$ from the 5 echo times in each measurement. This should mean that effects due to temperature, flow and haemolysis can be ignored, so that the $\mathrm{s}_{2}$ prediction depends only on the echo time splitting. This $\mathrm{K}_{0}$ value was then converted to an $\mathrm{sO}_{2}$ using the values in Figure 5.14, which describe the linear relationship between $K_{0}$ and $\left(1-\mathrm{sO}_{2}\right)^{2}$ at this field and haematocrit. In effect, this is the same as the 'Approach 3 ' trained method described by Varghese, where the 'nuisance parameters' are found from the training data, which are the slope parameter in Table 5.2. The short echo times used in these experiments, particularly the $1 \mathrm{~ms}$ time, should allow this method to better estimate $T_{20}$, while the wide range of echo times also provides a larger splitting, allowing for better estimation of $K_{0}$.

This method was applied to data from the $14 \mathrm{MHz}$ experiment, shown in Figure 5.18. The section of the experiment where the $T_{2}$ and optical sensor $\mathrm{s}_{2}$ values were extracted for analysis (effectively training data) is shown in grey. For comparison, a quadratic $\mathrm{R}_{2} / \mathrm{sO}_{2}$ calibration was also calculated using the value of $\mathrm{K}$ found above in subsection 5.4.2 and is shown in red.

This demonstrates that the $\mathrm{sO}_{2}$ calculated by the parametric method tracks the optical sensor measurement well, and is not affected by the background changes in $T_{2}$. It also shows that the quadratic calibration curve follows the optical sensor data, but is significantly biased towards lower $\mathrm{sO}_{2}$ as the experiment continues, due to the background decrease in $T_{2}$. The background decrease is shown by the decreasing $T_{20}$ line, which decreases from $305 \mathrm{~ms}$ to $250 \mathrm{~ms}$. Interestingly, the spikes where sudden changes in oxygenation occured (e.g. minutes 140,225) have a different shape in the NMR data to the optical sensor data. This could indicate that there is some sort of change in the $\mathrm{s}_{2}$ as the blood travels between the optical sensor and the probe.

The data also shows that the size of the splitting is not affected by the haemolysis - increased concentrations of haemoglobin in plasma could be expected to 


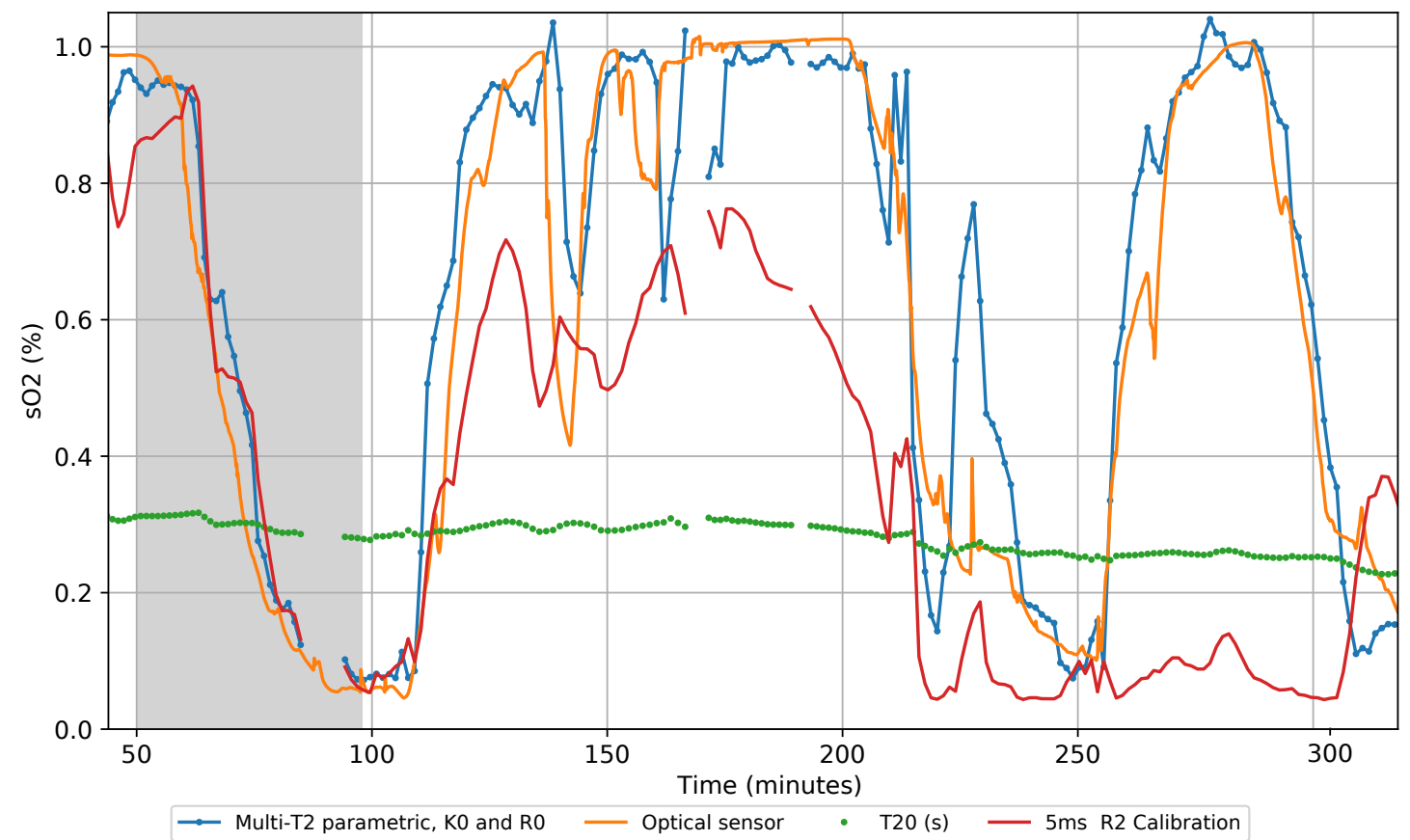

Figure 5.18: Back-predicted $\mathrm{sO}_{2}$ from $\mathrm{NMR}$ measurements at $14 \mathrm{MHz}$, using data highlighted in grey as 'training' data

decrease the susceptibility differences between the blood cell cytoplasm and the plasma, causing the strength of the $T_{2}$ shortening effect to decrease. This would lead to a decreased $\mathrm{K}_{0}$ for the same $\mathrm{sO}_{2}$, and therefore predict a higher $\mathrm{sO}_{2}$. This does not appear to be the case, for example at minute 250, where the optical and NMR data are still in good agreement, suggesting that the free haemoglobin is not at a high enough level to affect the field inhomogeneity.

\subsection{Summary}

These continuous flow experiments show that changes in $\mathrm{T}_{2}$ due to $\mathrm{sO}_{2}$ still occur at low field, with the size of the change proportional to $B_{0}$ squared. While the temperature and flow effects in the experiments may need to better controlled, the results still appear to follow the trends shown in the literature. Repeating these experiments with more samples of blood will also be needed to confirm these results, as they come from one sample at each field strength. Additionally, we have found evidence that blood was breaking down during the course of the experiments, suggesting that the $T_{2}$ could be sensitive to this process. By using the dependence of the $T_{2}$ change on CPMG echo time, the effect due to $\mathrm{sO}_{2}$ was 
isolated to provide a more robust method of measuring $\mathrm{sO}_{2}$. 


\section{Chapter 6}

\section{Models for $T_{2}$ relaxation enhancement}

As discussed in section 2.8, researchers have developed a number of models to explain the decrease in $T_{2}$ of the water protons in blood. Thulborn originally introduced the Luz-Meiboom equation to describe the decrease in $T_{2}$ and its dependence on the CPMG echo time [23]. Another model has been proposed by Jensen and Chandra, which describes the dephasing of water protons that diffuse through areas of magnetic field inhomogeneities [22]. Generally, the two models predict the same behaviour at short echo times $1 \mathrm{~ms}$ to $3 \mathrm{~ms}$ and at long echo times (>20 ms) [20], but in the intermediate range, the two models give different values for the decrease in $T_{2}$ as echo time changes. Additionally, experiments comparing the two models have found that at clinical MRI fields (1.5 T and up), both models provide good agreement with experimental data $[26,27,28,31]$ Because of this, the Luz-Meiboom equation is typically used when analysing data, as it is simpler than the diffusion model.

As another component of this research, the agreement between these models and data collected at lower fields has been tested to investigate which model is more effective at lower field.

\subsection{Theory}

The Luz-Meiboom equation (Equation 2.12) is typically used to describe the decrease in $\mathrm{T}_{2}$ due to changes in $\mathrm{sO}_{2}$. It usually provides good agreement with experimental data at different echo times, but it does not provide a good description of the underlying physical system - for example, what the exchange time actually 
Table 6.1: Previous studies of $T_{2}$ shortening and dependence on exchange time

\begin{tabular}{|llll|}
\hline Author & Year & Field $(\mathrm{T})$ & $\tau_{e x}(\mathrm{~ms})$ \\
\hline Thulborn & $1982[23]$ & 4.2 & 0.6 \\
Gomori & $1987[18]$ & 0.94 & $9.1 \pm 0.1$ \\
Bryant & $1990[17]$ & 1.4 & 10 \\
Brooks & $1995[19]$ & 1.0 & 3.4 \\
Meyer & $1995[29]$ & 4.7 & 1 \\
Golay & $2001[43]$ & 1.5 & 2.5 \\
Stefanovic & $2004[26]$ & 1.5 & $3.0 \pm 0.2$ \\
Chen & $2009[27]$ & 3 & $1.67 \pm 0.01$ \\
Gardener & $2010[28]$ & 2.35 & $4.4 \pm 0.4$ \\
Gardener & $2010[28]$ & 7 & $4.4 \pm 2.1$ \\
Varghese & $2017[35]$ & 1.5 & $5.0 \pm 1.1$ \\
\hline
\end{tabular}

measures is not clear.

In the literature, there are a range of values for the exchange time (Table 6.1), ranging from $0.6 \mathrm{~ms}$ to $9.1 \mathrm{~ms}$. While this has been attributed to using different ranges of echo time in the CPMG experiments, which can cause the fitting to become biased, many of these do not agree with values for the rate of transmembrane exchange when measured by other NMR methods (Typically $>10 \mathrm{~ms}$ [50]). This suggests that the exchange time parameter $\left(\tau_{e x}\right)$ is not necessarily due to exchange across the cell membrane.

In their paper, Jensen and Chandra study this effect using the weak-field approximation [22]. Using an exponential decay to approximate the correlation function $K(t)$, they derive a formula similar to the Luz-Meiboom equation for $T_{2}$ as a function of echo time $\left(t_{e c}\right)$. (Equation 2.14, restated here). In this formula, $\tau$ is the correlation time, and $K_{0}$ is the variance of the magnetic field.

$$
\begin{gathered}
K(t)=K_{0} e^{-t / \tau} \\
\frac{1}{T_{2}}=\frac{1}{T_{20}}+\gamma^{2} K_{0} \tau\left(1-\frac{2 \tau}{t_{e c}} \tanh \frac{t_{e c}}{2 \tau}\right)
\end{gathered}
$$

Jensen and Chandra also proposed a different approximation for $K(t)$ (Equation 6.1) which considers the diffusion of water molecules around microscopic field inhomogeneities with a size $r_{c}$. This new correlation function is intended to give the approximated correlation function more realistic asymptotic behaviour 
for diffusion than the exponential decay in Equation 2.13. Applying this correlation function gives Equation 6.2 for the $T_{2}$ dependence on echo time.

$$
\begin{gathered}
K(t) \approx G_{0}\left(1+\frac{4 D t}{r_{c}^{2}}\right)^{-3 / 2} \\
\frac{1}{T_{2}}=\frac{1}{T_{20}}+G_{0} \frac{\gamma^{2} r_{c}^{2}}{2 D} F\left(\frac{2 D t_{e c}}{r_{c}^{2}}\right) \\
\text { where } F(x)=\frac{1}{\sqrt{\pi}} \int_{0}^{\infty} \frac{e^{-y}}{\sqrt{y}}\left[1-\frac{1}{x y} \tanh x y\right] \mathrm{d} y
\end{gathered}
$$

Here, $t_{e} c$ is the echo time, $G_{0}$ is the mean squared magnitude of field inhomogeneities, $r_{c}$ is their characteristic length scale (i.e. the size of the red blood cells), and $D$ is the diffusion coefficient of water protons. In studies comparing the Luz-Meiboom and Jensen-Chandra equations, it has been shown that both agree adequately with experimental data, but that the Jensen and Chandra model is better. To qualitatively show the different predictions, the quantity $\frac{r_{c}^{2}}{2 D}$ can be looked at as a time (as it has the dimensions of time). By setting this time equal to the exchange time in the Luz-Meiboom equation, the two models can be compared, as shown in Figure 6.1 The two equations give slightly different shaped curves for the dependence of $T_{2}$ on echo time, and this different shape should be reflected in the agreement with the experimental data here.

\subsection{Experimental Setup}

To better map out the dependency of $T_{2}$ on echo time, a wider range of echo times than the 5 used in the other continuous experiments in chapter 5 was required. In this series of experiments, a new pulse sequence was written to sequentially measure multiple CPMG experiments using a range of echo times specified by the user. The pulse sequence supports either using a linear range of echo times, or loading a file with the desired echo times. A list of 13 times between $0.5 \mathrm{~ms}$ to $20 \mathrm{~ms}$ was used, with more closely spaced samples in the region between $1 \mathrm{~ms}$ to $5 \mathrm{~ms}$, as this is where the differences in the shape of the two predicted curves are most visible. To ease processing, the number of echoes in each CPMG experiment is the same. This presented a problem with measuring longer echo times, for example, 200 echoes at $20 \mathrm{~ms}$ requires an experiment time of $4 \mathrm{~s}$. Overall, the experiment with 13 echo times, and a $T_{R}$ of $5 \mathrm{~s}$ takes 5 minutes. 


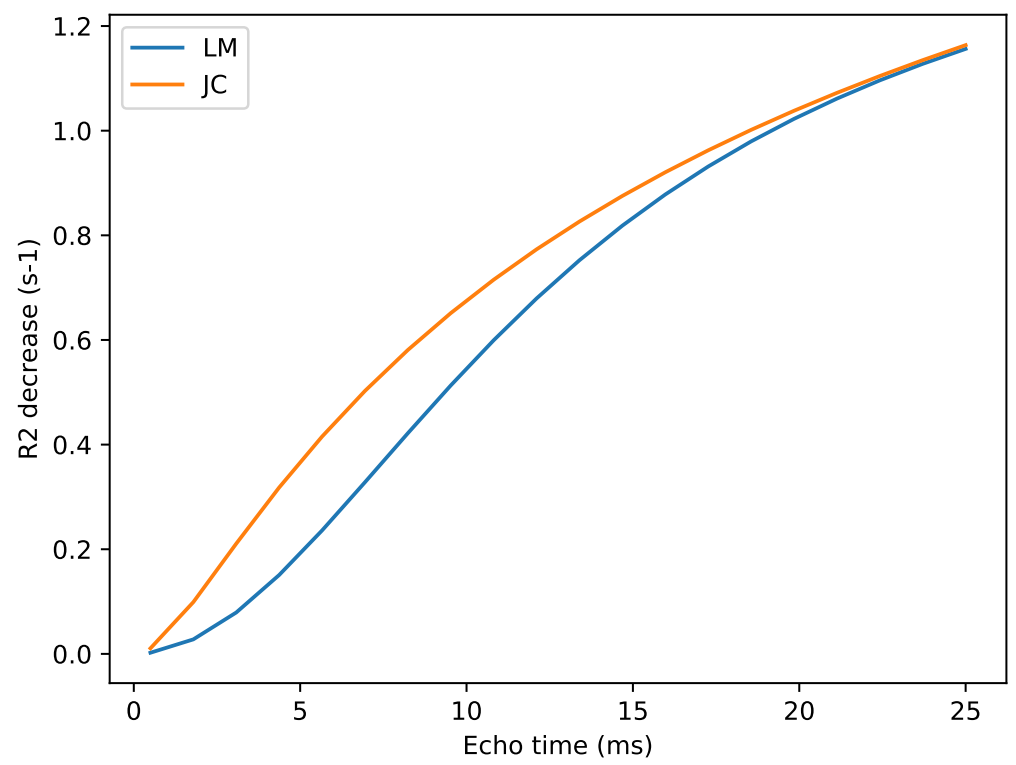

Figure 6.1: Different predictions of Luz-Meiboom and Jensen Chandra models. Parameters used are $G_{0}=1 \times 10^{-14} \mathrm{~T}^{2}, K_{0}=0.59 \times 10^{-14} \mathrm{~T}^{2}$ (to approximate the same relaxation effect size), $\tau_{e x}=4 \mathrm{~ms}, r_{c}=4 \mu \mathrm{m}, \mathrm{D}=2 \times 10^{3} \mu \mathrm{m}^{2} \mathrm{~s}^{-1}$. The parameter $\tau_{e x}=\frac{r_{c}^{2}}{2 D}=4 \mathrm{~ms}$.

These experiments were completed during the continuous flow experiments, with experiments done at each field strength. Each experiment was repeated right afterwards, and the two $T_{2}$ values were averaged.

Because of the difficulties found when trying to maintain intermediate levels of blood oxygenation (e.g. in the stopped flow experiments in chapter 4), experiments were done using blood in a deoxygenated state. Deoxygenated blood is typically used in the literature, and creates stronger contrast in the $T_{2}$ effect, due to the increased field inhomogeneities.

\section{Analysis method}

To compare the two models, the approach of Stefanovic and Pike at $1.5 \mathrm{~T}$ [26] was used. $T_{2}$ values were calculated for each echo time using least squares fitting of the phased and integrated echo amplitudes with a monoexponential decay. To remove the effect of the fast decaying signal from the tube surrounding the blood, the first $40 \mathrm{~ms}$ of the CPMG decay is ignored in the fitting. Data from after $1 \mathrm{~s}$ was also excluded, as the signal from the blood had generally decayed by this time, leaving only noise that increased fitting uncertainty. The average of $T_{2}$ values from 
the two experiments was used to fit to constrained versions of equations 2.14 and 6.2 (where either $\tau_{e x}$ or $r_{c}$ are held constant). In this constrained fitting process, the $\tau_{e x}$ was set to $3.0 \mathrm{~ms}$, and $r_{c}$ set to $4.3 \mu \mathrm{m}$, values reported by Stefanovic from studies at $1.5 \mathrm{~T}$ [26], the lowest field this sort of experiment has been done in the literature. Theory suggests that these should be field independent, but a range of $\tau_{e x}$ and $r_{c}$ values have been found in the literature. Their experiments typically held $T_{20}$ constant in the constrained models, but this would not have allowed for changes in intrinsic $T_{2}$ due to haemolysis or other changes in the state of the blood which occured in these experiments (subsection 5.4.3, so this was used as a second fitted parameter. In all fits, the diffusion coefficient of water was assumed to be $2.0 \mu \mathrm{m}^{2} \mathrm{~ms}^{-1}$. The results from the constrained fit were then used as initial guesses for an unconstrained least squares fit to the formulae, where all three parameters were allowed to vary. This was needed to ensure the curve fitting procedure converged. Finally, the Sum-of-Squared-Residuals (SSR) was calculated to give a quantitative measure of the model's agreement with the measured data.

\subsection{Results}

\subsection{1 $T_{2}$ Measurement}

CPMG decays were measured for blood in the continuous flow setup at each of the field strengths used in those experiments. The oxygenation was logged by optical sensor, and found to vary $<5 \%$ over the experiment. The same flow rate from the continuous flow experiments was maintained using the screw clamp, which meant that the blood did not have the chance to settle and that the temperature was relatively stable during the 5 minutes of the experiment (within $2{ }^{\circ} \mathrm{C}$ ).

To obtain a $T_{2}$ value for each echo time, the echo intensities were fit to a monoexponential decay, as discussed in section 2.3. An example of this is shown in Figure 6.2, the section of the decay used for fitting is also marked.

With the exception of the fast decay from the plastic tube at the start of the CPMG experiment, the log of the echo intensities follow a linear trend, which confirms that the blood is not separating, and that the shape of the decay is not significantly affected by flow. 


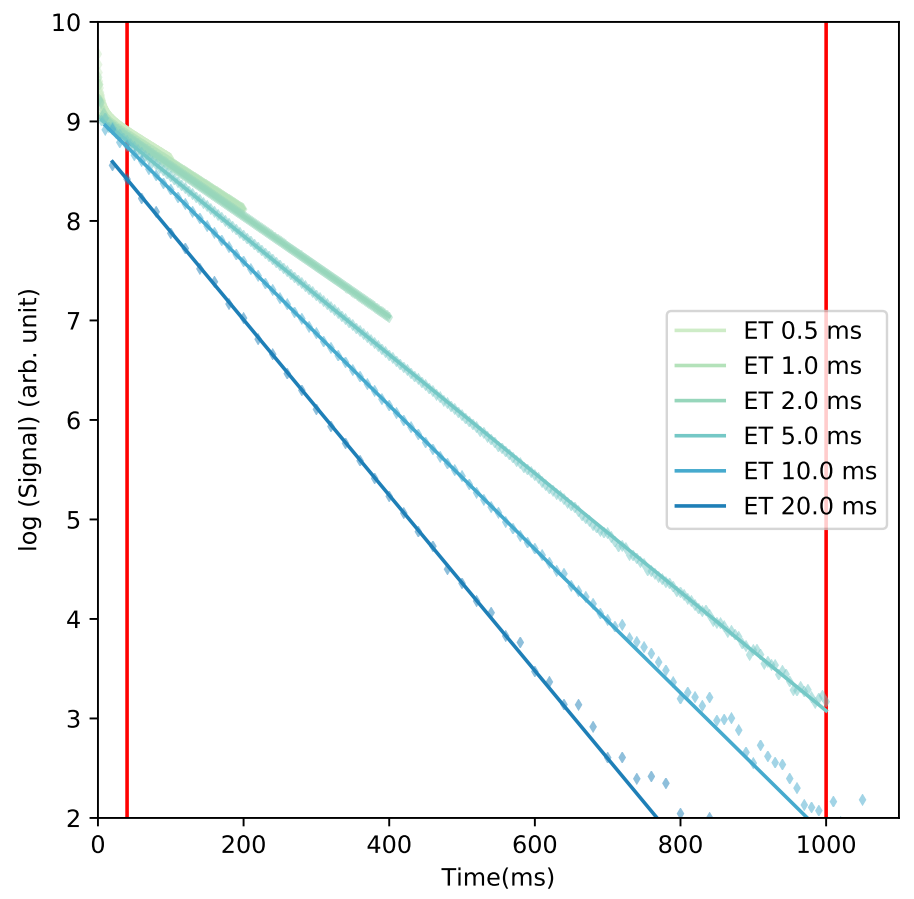

Figure 6.2: CPMG decays measured at the field of $20 \mathrm{MHz}$, at the different echo times. Red lines indicate data points used for fitting

\subsubsection{Model fitting}

Values of $T_{2}$ measured at each echo time were then used as inputs for fitting equations 2.14 and 6.2. Errors from the $T_{2}$ fitting are on the order of $2 \mathrm{~ms}$. The resulting best fit curves for both equations are shown in Figure 6.3, while the parameters are included in Table 6.2. 
Table 6.2: Best fit values to the experimental data at different fields, shaded columns indicate fixed parameters in fitting. Note that the $T_{20}$ values are dependent on the state of the blood in the flow circuit, so are not necessarily reflective of the diffusion/exchange effects.

\begin{tabular}{|c|c|c|c|c|c|c|c|c|}
\hline \multirow{3}{*}{ Field } & \multicolumn{3}{|c|}{ Constrained LM } & \multirow{3}{*}{$\begin{array}{l}\text { SSR } \\
\mathrm{ms}^{2}\end{array}$} & \multicolumn{4}{|c|}{ Unconstrained LM } \\
\hline & $T_{20}$ & $K_{0}$ & $\tau_{e x}$ & & $T_{20}$ & $K_{0}$ & $\tau_{e x}$ & SSR \\
\hline & $\mathrm{ms}$ & $10^{-14} \mathrm{~T}^{2}$ & $\mathrm{~ms}$ & & $\mathrm{~ms}$ & $10^{-14} \mathrm{~T}^{2}$ & $\mathrm{~ms}$ & $\mathrm{~ms}^{2}$ \\
\hline $40 \mathrm{MHz}$ & $227 \pm 4.2$ & $7.081 \pm 0.162$ & 3.00 & 3733 & $248 \pm 5.8$ & $8.445 \pm 0.311$ & $2.08 \pm 0.11$ & 1199 \\
\hline $20 \mathrm{MHz}$ & $219 \pm 1.6$ & $2.832 \pm 0.022$ & 3.00 & 277 & $210 \pm 1.9$ & $2.459 \pm 0.048$ & $3.55 \pm 0.08$ & 498 \\
\hline $14 \mathrm{MHz}$ & $297 \pm 0.5$ & $0.868 \pm 0.005$ & 3.00 & 410 & $298 \pm 0.5$ & $0.990 \pm 0.020$ & $2.30 \pm 0.08$ & 183 \\
\hline $10 \mathrm{MHz}$ & $294 \pm 1.5$ & $0.473 \pm 0.023$ & 3.00 & 616 & $305 \pm 3.3$ & $0.654 \pm 0.062$ & $1.96 \pm 0.18$ & 89 \\
\hline $5 \mathrm{MHz}$ & $277 \pm 2.2$ & $0.392 \pm 0.025$ & 3.00 & 443 & $283 \pm 3.2$ & $0.588 \pm 0.092$ & $1.81 \pm 0.30$ & 177 \\
\hline
\end{tabular}

\begin{tabular}{|c|c|c|c|c|c|c|c|c|}
\hline \multirow[b]{2}{*}{ Field } & \multicolumn{4}{|c|}{ Constrained JC } & \multicolumn{4}{|c|}{ Unconstrained JC } \\
\hline & $\begin{array}{l}T_{20} \\
\mathrm{~ms}\end{array}$ & $\begin{array}{c}G_{0} \\
10^{-14} \mathrm{~T}^{2}\end{array}$ & $\begin{array}{c}r_{c} \\
\mu \mathrm{m}\end{array}$ & $\begin{array}{l}\text { SSR } \\
\mathrm{ms}^{2}\end{array}$ & $\begin{array}{l}T_{20} \\
\mathrm{~ms}\end{array}$ & $\begin{array}{c}G_{0} \\
10^{-14} \mathrm{~T}^{2}\end{array}$ & $\begin{array}{c}r_{c} \\
\mu \mathrm{m}\end{array}$ & $\begin{array}{l}\mathrm{SSR} \\
\mathrm{ms}^{2}\end{array}$ \\
\hline $40 \mathrm{MHz}$ & $253 \pm 5.2$ & $9.985 \pm 0.217$ & 4.30 & 844 & $272 \pm 8.6$ & $11.643 \pm 0.636$ & $3.58 \pm 0.19$ & 120 \\
\hline $20 \mathrm{MHz}$ & $225 \pm 1.7$ & $3.932 \pm 0.031$ & 4.30 & 56 & $220 \pm 2.4$ & $3.588 \pm 0.105$ & $4.65 \pm 0.12$ & 34 \\
\hline $14 \mathrm{MHz}$ & $299 \pm 0.5$ & $1.227 \pm 0.007$ & 4.30 & 88 & $300 \pm 0.6$ & $1.393 \pm 0.044$ & $3.70 \pm 0.12$ & 35 \\
\hline $10 \mathrm{MHz}$ & $301 \pm 1.9$ & $0.748 \pm 0.036$ & 4.30 & 181 & $309 \pm 4.4$ & $1.022 \pm 0.161$ & $3.29 \pm 0.38$ & 13 \\
\hline $5 \mathrm{MHz}$ & $279 \pm 2.3$ & $0.571 \pm 0.036$ & 4.30 & 302 & $291 \pm 5.7$ & $1.364 \pm 0.485$ & $2.32 \pm 0.48$ & 34 \\
\hline
\end{tabular}


As expected, all of the curves show that longer echo times create a larger decrease in the $T_{2}$. The magnitude of the effect becomes much weaker at lower field - for example, at $40 \mathrm{MHz}$ the $T_{2}$ drops $200 \mathrm{~ms}$ (from $270 \mathrm{~ms}$ to $70 \mathrm{~ms}$ ) while at 10 $\mathrm{MHz}$, the $T_{2}$ drop is $60 \mathrm{~ms}$ (300 ms to $245 \mathrm{~ms}$ ).

The fitted curves show relatively good agreement with the experimental data, particularly in $20 \mathrm{MHz}$ (b) and $14 \mathrm{MHz}$ (c) and $10 \mathrm{MHz}$ (d) experiments. Generally, the unconstrained fits (solid lines) perform better than the constrained fits, this is shown again by the lower SSR values in Table 6.2. This is expected due to the extra free parameter in the curve fitting procedure.

In the $40 \mathrm{MHz}$ experiment, there is a larger deviation between the data points and best fit curves, particularly at short echo times. The start of the curve is strongly influenced by the $T_{20}$ term, which reflects the intrinsic relaxation at infinitely short refocusing times (i.e. removing the effect of exchange or diffusion.) This means that the $T_{20}$ term may be underestimated in this experiment, which would also explain the larger uncertainty relative to the other field strengths.

The results also suggest that the diffusion model produces better agreement than the Luz Meiboom model. This is shown by the decreased SSR in all cases, when comparing results at the same field strength.

The $T_{20}$ values tend to be between $250 \mathrm{~ms}$ to $310 \mathrm{~ms}$ for all except the $20 \mathrm{MHz}$ experiments which are at $220 \mathrm{~ms}$. This may be due to the state of the blood in the flow circuit, because as mentioned in chapter 5 , experiments at $20 \mathrm{MHz}$ were run after the $40 \mathrm{MHz}$, using the same blood sample. This is unlikely to be correlated to the flow rate, as the $5 \mathrm{MHz}$ results were measured at a similarly fast speed.

Additionally, trends can also be seen in the $K_{0}$ and $G_{0}$ values. These values reflect the strength of the magnetic field inhomogeneities, which is dependent on the oxygen saturation, haematocrit and also on the magnetic field strength. While this property was examined in chapter 5 , to investigate the dependence on oxygen saturation, in these samples where oxygen saturation is uniformly low the $K_{0}$ and $G_{0}$ terms should have a dependence on the field strength. The $K_{0}$ and $G_{0}$ values for the unconstrained fits follow a decreasing trend, although the $G_{0}$ increases at the $5 \mathrm{MHz}$ experiment, which may be related to the smaller $r_{c}$. The values of $K_{0}$ and $G_{0}$ from the unconstrained fits are plotted in Figure 6.4 as a log-log plot to determine their dependence on field strength. Ignoring the $5 \mathrm{MHz}$ data point, the other field strengths follow a linear trend on this graph. The slope of the line is 1.75 (not enough data to find uncertainty), which is close to the expected value of 2 for a quadratic dependence. Fitting these points to a quadratic relationship 
(a) $40 \mathrm{MHz}: \mathrm{sO}_{2} 2 \%$, Hct $0.33, \mathrm{v}=1.0 \mathrm{~cm} / \mathrm{s}$

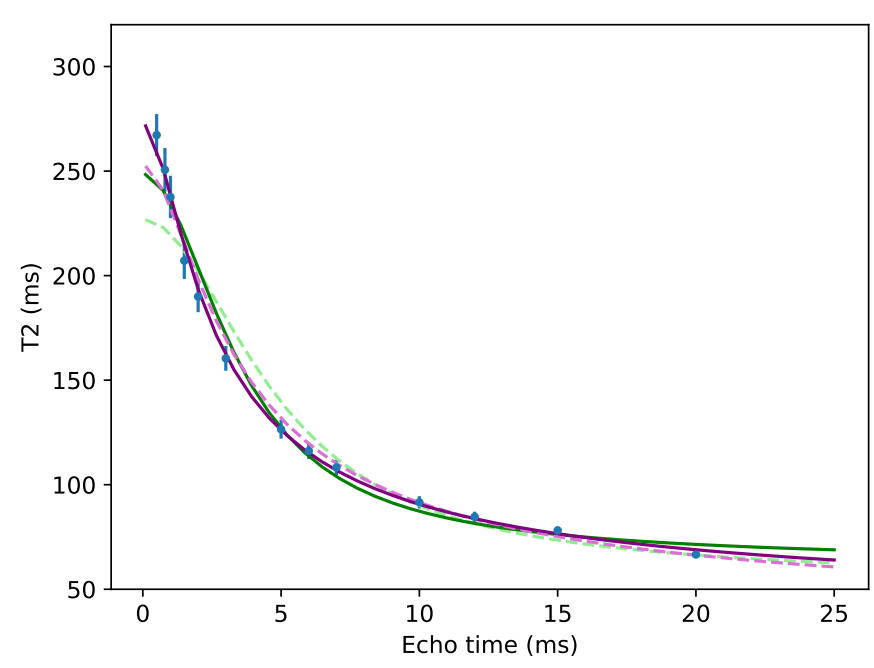

(c) $14 \mathrm{MHz}: \mathrm{sO}_{2} 5 \%$, Hct $0.34, \mathrm{v}=1.1 \mathrm{~cm} / \mathrm{s}$

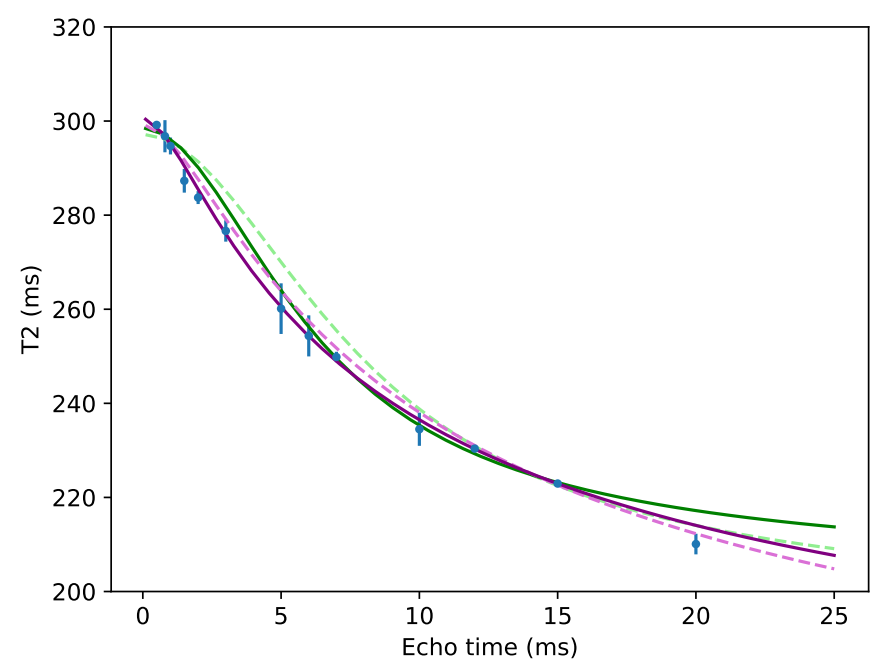

(e) 5 MHz: $s \mathrm{O}_{2} 7 \%$, Hct $0.35, \mathrm{v}=2.5 \mathrm{~cm} / \mathrm{s}$

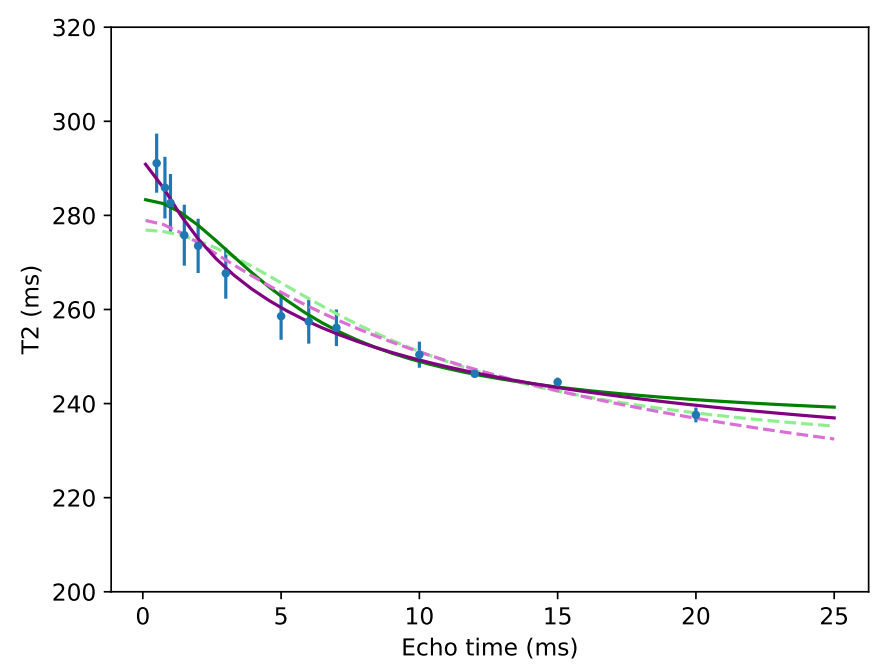

(b) $20 \mathrm{MHz}:\left(\mathrm{sO}_{2} 1 \%\right.$, Hct $0.38, \mathrm{v}=1.9 \mathrm{~cm} / \mathrm{s}$

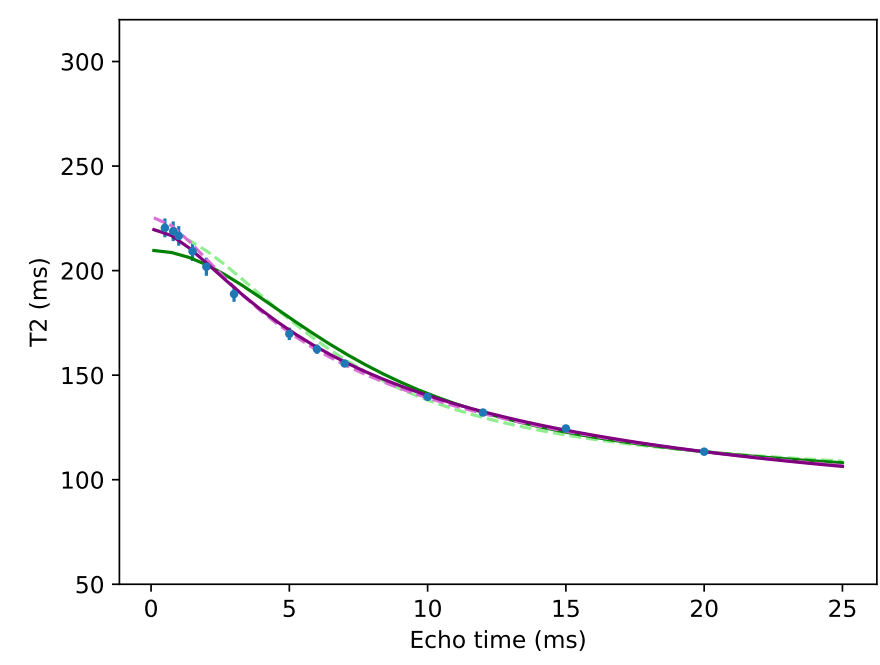

(d) $10 \mathrm{MHz}: \mathrm{sO}_{2} 2 \%$, Hct $0.37, \mathrm{v}=1.6 \mathrm{~cm} / \mathrm{s}$

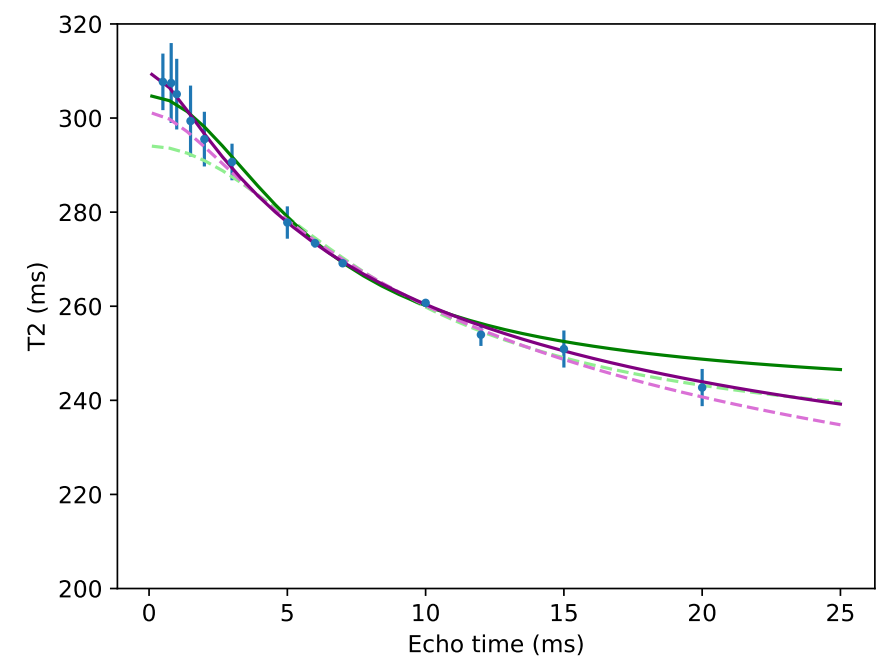

Error bars show standard error $(n=2)$. Best fit from Luz-Meiboom (Green) and diffusion model (Purple) are also shown. Dashed lines show constrained fits, while solid lines are unconstrained. Note different scale for $40 \mathrm{MHz}$ and $20 \mathrm{MHz}$ 


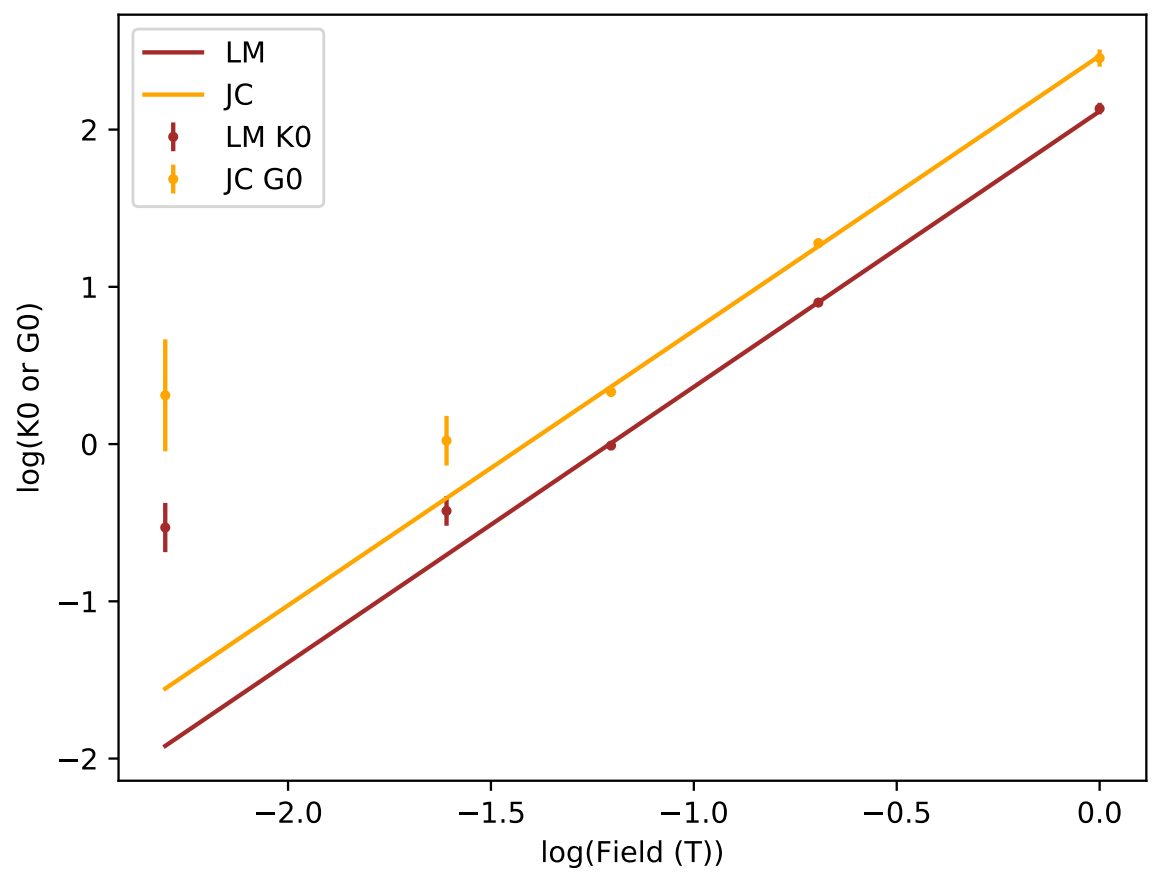

Figure 6.4: Field dependence of $G_{0}$ and $K_{0}$. Lines of best fit both have slope $=1.75$

yields $K_{0}=(8.56 \pm 0.34) \times 10^{-14} B_{0}^{2}$ and $G_{0}=(11.8 \pm 0.7) \times 10^{-14} B_{0}^{2}$.

\subsection{Discussion}

These results show that the diffusion model of Jensen and Chandra tends to agree better with the measured data, even at low fields. This is shown by the decreased SSR - the unconstrained JC fits provides much lower residuals than all other methods. However, the curves / predictions of the LM model tend to be within $10 \mathrm{~ms}$ of the experimental data, which means that both models are probably adequate for predicting $T_{2}$. This is the same conclusion reached by Stefanovic and Pike and Chen and Pike, who found that the Luz-Meiboom exchange model provides acceptable accuracy at $1.5 \mathrm{~T}$ and at $3 \mathrm{~T}$ respectively.

Additionally, with the exception of the $20 \mathrm{MHz}$ results (where experiments were run after the $40 \mathrm{MHz}$ run), the unconstrained fits produce concordant results of $\tau_{e x}=(2.17 \pm 0.06) \mathrm{ms}$ and $r_{c}=(3.58 \pm 0.08) \mu \mathrm{m}$ (weighted mean across field strengths). This value for $r_{c}$ is within the range of red blood cell size, although it is smaller than the value found by Stefanovic $(4.3 \mu \mathrm{m})$ [26], and larger than the value found by Chen $(2.7 \mu \mathrm{m})$ [27].

This value for the exchange time is also within the range of results in the liter- 
ature, although they do not correspond with the values measured at low field by Gomori [18]. This may be due to the long echo times they used, e.g. Gomori used two echo times of $32 \mathrm{~ms}$ and $64 \mathrm{~ms}$ at $0.94 \mathrm{~T}$ to get very short $T_{2}$ decays. These long echo times were used because they were unable to get a good estimate of $T_{2}$ shortening at short echo times due to large uncertainties. Looking at these long echo times may lead to greater sensitivity to exchange processes, and in particular, the $9.1 \mathrm{~ms}$ exchange time found by Gomori aligns well with literature values from permeability studies of the red blood cell membrane, which found values on the order of $10 \mathrm{~ms}$ [50].

Changes in the value of $r_{c}$ when moving from $40 \mathrm{MHz}$ to $20 \mathrm{MHz}$ are interesting, as it is known to be the same sample of blood. This could reflect changes in the state of the blood, due to being cycled through the flow circuit for 7 hours. More precisely, the fitted parameter in Equation 6.2 is actually $\frac{r_{c}^{2}}{2 D}$, and it is possible that an increase in the fitted $r_{c}$ could also be due to a decrease in $D$, the diffusion coefficient. It is also possible that the decrease is due to experimental factors, such as a change in the shim of the magnet when moving fields, which could affect the spread of $T_{2}$ values.

The straight lines in Figure 6.4 suggest that $K_{0}$ and $G_{0}$ follow a power law, although the dependence here is on $B_{0}{ }^{1.75}$, rather than the 2.1 found in the continuous flow experiment (subsection 5.3.7). 1.75 is still close to the 2 predicted by theory [22, Eq. 52-54]. Extrapolating this to $1.5 \mathrm{~T}$ predicts a value of $G_{0}=$ $26.7 \times 10^{-14} \mathrm{~T}^{2}$. This is about half of what is predicted using the results of Stefanovic, which (although based on studying changes in $\mathrm{sO}_{2}$ ) can be used to find $G_{0}=(45.00 \pm 0.51) \times 10^{-14}\left(\mathrm{sO}_{2}\right)^{2}=40.6 \times 10^{-14} \mathrm{~T}^{2}$ when using $\mathrm{sO}_{2}=0.03$, the average value from our experiments. While the difference is significant, it is important to note that the variation between the oxygenations and haematocrit used at the different field strengths was not taken into account when fitting the data to find $G_{0}$. Jensen and Chandra provide theoretical expressions for the value of $G_{0}$ using the susceptibility change in the RBC and the haematocrit in their diffusion model [22]. Multiple studies [26, 27, 28] have found that these provide reasonable agreement with experimental data. Further investigation into the effect of these parameters could test the applicability of this diffusion model at lower fields.

One possible source of error in these results is the effect of flow on the $T_{2}$ measurements. It was shown in subsection 5.2.1 that low amounts of flow through the circuit can cause slight decreases in the measured $T_{2}$, without causing significant changes to the monoexponential decay in the CPMG experiment. Because 
the $T_{2}$ values are fit to the Luz-Meiboom and Jensen Chandra equations, this may cause the $T_{20}$ term to become smaller. However, the doped water flow experiments showed that there was only a small change that was dependent on echo time, with a slight decrease at a $20 \mathrm{~ms}$ echo time when compared to the $1 \mathrm{~ms}$ echo time points. In these variable echo time experiments, lower $T_{2}$ values at longer echo times, would lead to an overestimation in the magnitude of the $T_{2}$ shortening effect. This would affect the $K_{0}$ and $G_{0}$ terms, as they would be split further apart. The flow rates in these experiments were only $1 \mathrm{~cm} / \mathrm{s}$ to $2 \mathrm{~cm} / \mathrm{s}$ in these experiment, which means that this should not be a significant effect. Additionally, the flow rate has been assumed to be constant over the 5-minute course of the experiment. This is relatively short compared to the longer-term trends which were seen in the continuous flow experiments, so this should not have a significant effect.

As both models have acceptable predictions for the shape of the echo time curve, these results cannot provide more evidence of the actual physical mechanism of the $T_{2}$ shortening effect. To be able to determine this, other experimental NMR techniques would need to be used such as Diffusion-Diffusion Correlation Spectroscopy (DDCOSY) or Diffusion-Exchange Spectroscopy (DEXSY) that could detect this process directly. Other researchers have also used paramagnetic contrast agents to probe exchange between intra- and extracellular pools in blood [51]. Matwiyoff [52] proposed that as the field decreased below 1.5 T, the exchange time would increase as the mechanism moved from mainly diffusion to mainly exchange. This is the opposite to the trend observed here, where there is a slightly decreasing or constant trend in the exchange time, as field strength decreases.

\subsection{Summary}

The results in this chapter confirm that both models that describe this effect produce good agreement with the data measured at lower fields. While fitting the Jensen and Chandra model produced lower residuals, the differences between the two models were small when compared to the size of the $T_{2}$ change due to the echo time. Because of the variations between the measurements at different fields, with different samples of blood at each field, different amounts of time in the flow circuit, and variations in factors such as flow rate and oxygen saturation, it is difficult to make strong conclusions about the parameters underlying the models. This would require more data, with different samples of blood and 
better control of these experimental factors. These findings are in agreement with previous studies in the literature. 


\section{Chapter 7}

\section{Conclusions and Future Work}

These results confirm that changes in $T_{2}$ due to blood oxygenation can be detected at low fields in this in-vitro system. The continuous flow experiments showed that this $T_{2}$ effect follows the trends predicted by the Luz-Meiboom equation, with the increase in $\mathrm{R}_{2}$ and $\mathrm{K}_{0}$ being proportional to $\left(1-\mathrm{sO}_{2}\right)^{2}$ and to $\mathrm{B}_{0}{ }^{2}$. While some experimental parameters will need to be better controlled in future experiments, the effects of temperature and flow rate do not cause significant uncertainty in the results. A decrease in $T_{2}$ also occured over the course of each experiment, and experiments using UV/Vis spectroscopy suggested this was due to haemolysis occurring in the blood. By applying the Luz-Meiboom model, measurements using different echo times were used to remove the effect of the background $T_{2}$ change in the blood samples, allowing for good tracking of the blood $\mathrm{sO}_{2}$.

In a separate series of experiments, the effect of echo time on the $T_{2}$ decrease was tested. The Luz-Meiboom equation worked well to describe this effect, although the Jensen and Chandra formula produced better agreement at all field strengths. This agrees with findings in the literature.

This series of experiments will need to be repeated to become more robust, as there is only a single blood sample used in each experiment for the 5 field strengths. Comparing the dependence of $T_{2}, R_{2}$ and $K_{0}$ on $s O_{2}$ across these samples and field strengths will provide more confidence in the accuracy of these experimental results. With more experiments, it will also be possible to investigate the effect of haematocrit, which has not been tested in this research. Experiments with concentrated or diluted blood samples will allow the effect of haematocrit to be isolated from the effect of field strength.

While the results show that changes in blood oxygenation can be detected, there may still be more challenges before this technique can be applied in-vivo. For 
example these experiments detected the $T_{2}$ change in blood, but an in-vivo system will need to detect the $T_{2}$ change in blood while in tissue. Creating a magnet system will also present difficulties, as it can be difficult to optimise parameters such as field strength, field homogeneity and the sweet spot size and depth in a portable NMR system. It will be important to know what compromises can be made in the design of a real system, while still maintaining good sensitivity to the $T_{2}$ contrast.

Other types of MRI contrast and techniques also have the potential to be applied in low field portable NMR systems. In high-field systems, techniques such as diffusion-weighted imaging can be used to detect the early signs of damage to the brain. Recently, the gradients produced by the NMR MOLE were used to provide accurate measurements of the diffusion coefficients in samples of liquid. The detection of perfusion is also a clinically valuable measurement that could be implemented with a low field system.

These results show that the $T_{2}$ changes in blood due to changes in blood oxygenation are visible at low field, and serve as a foundation for the future development of a new low field device. 


\section{Conference Attendance}

The work in this thesis was presented at:

- Y.C. Tzeng, D.G. Thomas, P. Galvosas, S. Obruchkov, P.D. Teal, Measurement of transverse relaxation properties of whole blood at low magnetic fields. The $14^{\text {th }}$ International Conference on Magnetic Resonance Microscopy, oral presentation, Halifax NS Canada, August 2017

- Dion G. Thomas, Petrik Galvosas, Yu-Chieh Tzeng, Paul D. Teal, Sergei Obruchkov, Measurement of transverse relaxation properties of whole blood at low magnetic fields. The $11^{\text {th }}$ Australian and New Zealand Society for Magnetic Resonance Conference, poster and short oral presentation, Kingscliff NSW Australia, December 2017 


\section{Bibliography}

[1] A. Abragam, Principles of Nuclear Magnetism. No. 32 in International Series of Monographs on Physics, New York: Oxford University Press, paperback ed., 1961.

[2] P. Callaghan, Principles of Nuclear Magnetic Resonance Microscopy. Oxford: Clarendon Press, revised ed. edition ed., Jan. 1994.

[3] R. W. Brown, Y.-C. N. Cheng, E. M. Haacke, M. R. Thompson, and R. Venkatesan, Magnetic Resonance Imaging: Physical Principles and Sequence Design. Hoboken, New Jersey: Wiley-Blackwell, 2 edition ed., June 2014.

[4] E. L. Hahn, "Spin Echoes," Physical Review, vol. 80, pp. 580-594, Nov. 1950.

[5] H. Y. Carr and E. M. Purcell, "Effects of Diffusion on Free Precession in Nuclear Magnetic Resonance Experiments," Physical Review, vol. 94, pp. 630-638, May 1954.

[6] S. Meiboom and D. Gill, "Modified Spin-Echo Method for Measuring Nuclear Relaxation Times," Review of Scientific Instruments, vol. 29, pp. 688-691, Aug. 1958.

[7] E. O. Stejskal and J. E. Tanner, "Spin Diffusion Measurements: Spin Echoes in the Presence of a Time-Dependent Field Gradient," The Journal of Chemical Physics, vol. 42, pp. 288-292, Jan. 1965.

[8] P. T. Callaghan and Y. Xia, "Velocity and diffusion imaging in dynamic NMR microscopy," Journal of Magnetic Resonance (1969), vol. 91, pp. 326-352, Feb. 1991.

[9] Y. Xia and P. T. Callaghan, "“One-shot" velocity microscopy: NMR imaging of motion using a single phase-encoding step," Magnetic Resonance in Medicine, vol. 23, pp. 138-153, Jan. 1992. 
[10] P. T. Callaghan, Translational Dynamics and Magnetic Resonance: Principles of Pulsed Gradient Spin Echo NMR. Oxford: Oxford University Press, 1 edition ed., Apr. 2014.

[11] J. E. Hall, Guyton and Hall Textbook of Medical Physiology. Philadelphia, PA: Elsevier, 13 ed., 2015.

[12] J. Toffaletti and W. G. Zijlstra, "Misconceptions in reporting oxygen saturation," Anesthesia and Analgesia, vol. 105, pp. S5-9, Dec. 2007.

[13] L. Pauling and C. D. Coryell, "The Magnetic Properties and Structure of Hemoglobin, Oxyhemoglobin and Carbonmonoxyhemoglobin," Proceedings of the National Academy of Sciences of the United States of America, vol. 22, pp. 210-216, Apr. 1936.

[14] O. Wieben, "Light Absorbance in Pulse Oximetry," in Design of Pulse Oximeters, Series in Medical Physics and Biomedical Engineering, Taylor \& Francis, Oct. 1997.

[15] V. Jain, O. Abdulmalik, K. J. Propert, and F. W. Wehrli, "Investigating the magnetic susceptibility properties of fresh human blood for noninvasive oxygen saturation quantification," Magnetic Resonance in Medicine, vol. 68, pp. 863-867, Sept. 2012.

[16] Z. Luz and S. Meiboom, "Nuclear Magnetic Resonance Study of the Protolysis of Trimethylammonium Ion in Aqueous Solution-Order of the Reaction with Respect to Solvent," The Journal of Chemical Physics, vol. 39, pp. 366-370, July 1963.

[17] R. G. Bryant, K. Marill, C. Blackmore, and C. Francis, "Magnetic relaxation in blood and blood clots," Magnetic Resonance in Medicine, vol. 13, pp. 133-144, Jan. 1990.

[18] J. M. Gomori, R. I. Grossman, C. Yu-Ip, and T. Asakura, "NMR Relaxation Times of Blood: Dependence on Field Strength, Oxidation State, and Cell Integrity," Journal of Computer Assisted Tomography, vol. 11, no. 4, p. 684, 1987.

[19] R. A. Brooks, J. Vymazal, C. D. Baumgarner, V. Tran, and J. W. M. Bulte, "Comparison of 2 relaxation in blood, brain, and ferritin," Journal of Magnetic Resonance Imaging, vol. 5, pp. 446-450, July 1995. 
[20] R. A. Brooks, F. Moiny, and P. Gillis, "On T2-shortening by weakly magnetized particles: The chemical exchange modelt," Magnetic Resonance in Medicine, vol. 45, pp. 1014-1020, June 2001.

[21] G. A. Wright, B. S. Hu, and A. Macovski, "Estimating oxygen saturation of blood in vivo with MR imaging at $1.5 \mathrm{~T}$," Journal of Magnetic Resonance Imaging, vol. 1, pp. 275-283, May 1991.

[22] J. Jensen and R. Chandra, "NMR relaxation in tissues with weak magnetic inhomogeneities," Magnetic Resonance in Medicine, vol. 44, pp. 144-156, July 2000 .

[23] K. R. Thulborn, J. C. Waterton, P. M. Matthews, and G. K. Radda, “Oxygenation dependence of the transverse relaxation time of water protons in whole blood at high field," Biochimica Et Biophysica Acta, vol. 714, pp. 265-270, Feb. 1982.

[24] H. Stadelmann, E. Muller, and K. Geibel, "Relaxation times for venous blood as a function of field strength," Electromedica (English Edition), vol. 59, no. 3, p. 82, 1991.

[25] M. Silvennoinen, C. Clingman, X. Golay, R. Kauppinen, and P. van Zijl, "Comparison of the dependence of blood $\mathrm{R} 2$ and $\mathrm{R} 2 *$ on oxygen saturation at 1.5 and 4.7 Tesla," Magnetic Resonance in Medicine, vol. 49, pp. 47-60, Jan. 2003.

[26] B. Stefanovic and G. B. Pike, "Human whole-blood relaxometry at 1.5T: Assessment of diffusion and exchange models," Magnetic Resonance in Medicine, vol. 52, pp. 716-723, Oct. 2004.

[27] J. J. Chen and G. B. Pike, "Human whole blood T2 relaxometry at 3 Tesla," Magnetic Resonance in Medicine, vol. 61, pp. 249-254, Feb. 2009.

[28] A. G. Gardener, S. T. Francis, M. Prior, A. Peters, and P. A. Gowland, "Dependence of blood R2 relaxivity on CPMG echo-spacing at 2.35 and $7 \mathrm{~T}$," Magnetic Resonance in Medicine, vol. 64, pp. 967-974, Oct. 2010.

[29] M.-E. Meyer, O. Yu, B. Eclancher, D. Grucker, and J. Chambron, "NMR relaxation rates and blood oxygenation level," Magnetic Resonance in Medicine, vol. 34, pp. 234-241, Aug. 1995. 
[30] J. M. Zhao, C. S. Clingman, M. J. Närväinen, R. A. Kauppinen, and P. C. van Zijl, "Oxygenation and hematocrit dependence of transverse relaxation rates of blood at 3T," Magnetic Resonance in Medicine, vol. 58, pp. 592-597, Sept. 2007.

[31] K. Grgac, W. Li, A. Huang, Q. Qin, and P. C. M. van Zijl, “Transverse water relaxation in whole blood and erythrocytes at 3T, 7T, 9.4T, 11.7T and 16.4T; determination of intracellular hemoglobin and extracellular albumin relaxivities," Magnetic Resonance Imaging, vol. 38, pp. 234-249, May 2017.

[32] Q. Qin, K. Grgac, and P. C. van Zijl, "Determination of whole-brain oxygen extraction fractions by fast measurement of blood T2 in the jugular vein," Magnetic Resonance in Medicine, vol. 65, pp. 471-479, Feb. 2011.

[33] Z. B. Rodgers, J. A. Detre, and F. W. Wehrli, "MRI-based methods for quantification of the cerebral metabolic rate of oxygen," Journal of Cerebral Blood Flow \& Metabolism, vol. 36, Feb. 2016.

[34] H. Lu and Y. Ge, "Quantitative evaluation of oxygenation in venous vessels using T2-Relaxation-Under-Spin-Tagging MRI," Magnetic Resonance in Medicine, vol. 60, pp. 357-363, Aug. 2008.

[35] J. Varghese, L. C. Potter, R. LaFountain, X. Pan, S. V. Raman, R. Ahmad, and O. P. Simonetti, "CMR-based blood oximetry via multi-parametric estimation using multiple T2 measurements," Journal of Cardiovascular Magnetic Resonance, vol. 19, p. 88, Nov. 2017.

[36] E. M. Haacke, S. Lai, J. R. Reichenbach, K. Kuppusamy, F. G. Hoogenraad, H. Takeichi, and W. Lin, "In vivo measurement of blood oxygen saturation using magnetic resonance imaging: A direct validation of the blood oxygen level-dependent concept in functional brain imaging," Human Brain Mapping, vol. 5, pp. 341-346, Jan. 1997.

[37] M. A. Fernández-Seara, A. Techawiboonwong, J. A. Detre, and F. W. Wehrli, "MR susceptometry for measuring global brain oxygen extraction," Magnetic Resonance in Medicine, vol. 55, pp. 967-973, May 2006.

[38] M. C. Langham, A. E. Rodríguez-Soto, N. Schwartz, and F. W. Wehrli, “In vivo whole-blood $\mathrm{T} 2$ versus $\mathrm{HbO} 2$ calibration by modulating blood oxygenation level in the femoral vein through intermittent cuff occlusion," Magnetic Resonance in Medicine, vol. 79, pp. 2290-2296, Apr. 2018. 
[39] J. R. Hess, "An update on solutions for red cell storage," Vox Sanguinis, vol.91, pp. 13-19, July 2006.

[40] Abbott Point of Care, "Cartridge and Test Information: pO2 and Calculated Oxygen Saturation," tech. rep., Abbott Park, IL US, Mar. 2013.

[41] B. Manz, A. Coy, R. Dykstra, C. D. Eccles, M. W. Hunter, B. J. Parkinson, and P. T. Callaghan, "A mobile one-sided NMR sensor with a homogeneous magnetic field: The NMR-MOLE," Journal of Magnetic Resonance, vol. 183, pp. 25-31, Nov. 2006.

[42] Y. Ma, A. J. Berman, and G. B. Pike, "The effect of dissolved oxygen on the relaxation rates of blood plasma: Implications for hyperoxia calibrated BOLD," Magnetic Resonance in Medicine, vol. 76, pp. 1905-1911, Dec. 2016.

[43] X. Golay, M. J. Silvennoinen, J. Zhou, C. S. Clingman, R. A. Kauppinen, J. J. Pekar, and P. C. van Zij, "Measurement of tissue oxygen extraction ratios from venous blood T2: Increased precision and validation of principle," Magnetic Resonance in Medicine, vol. 46, pp. 282-291, Aug. 2001.

[44] S. Portnoy, M. Osmond, M. Y. Zhu, M. Seed, J. G. Sled, and C. K. Macgowan, "Relaxation properties of human umbilical cord blood at 1.5 Tesla," Magnetic Resonance in Medicine, vol. 77, pp. 1678-1690, Apr. 2017.

[45] R. A. Malinauskas, "Plasma Hemoglobin Measurement Techniques for the In Vitro Evaluation of Blood Damage Caused by Medical Devices," Artificial Organs, vol. 21, pp. 1255-1267, Dec. 1997.

[46] V. F. Fairbanks, S. C. Ziesmer, and P. C. O'Brien, "Methods for measuring plasma hemoglobin in micromolar concentration compared.," Clinical Chemistry, vol. 38, pp. 132-140, Jan. 1992.

[47] S. O. Sowemimo-Coker, "Red blood cell hemolysis during processing," Transfusion Medicine Reviews, vol. 16, pp. 46-60, Jan. 2002.

[48] L. Vercaemst, "Hemolysis in cardiac surgery patients undergoing cardiopulmonary bypass: A review in search of a treatment algorithm," The Journal of Extra-Corporeal Technology, vol. 40, pp. 257-267, Dec. 2008.

[49] S. Portnoy, M. Seed, J. G. Sled, and C. K. Macgowan, "Non-invasive evaluation of blood oxygen saturation and hematocrit from $\mathrm{T} 1$ and $\mathrm{T} 2$ relaxation 
times: In-vitro validation in fetal blood," Magnetic Resonance in Medicine, vol. 78, pp. 2352-2359, Feb. 2017.

[50] M. D. Herbst and J. H. Goldstein, "A review of water diffusion measurement by NMR in human red blood cells," American Journal of Physiology - Cell Physiology, vol. 256, pp. C1097-C1104, May 1989.

[51] J. G. Li, G. J. Stanisz, and R. M. Henkelman, "Integrated analysis of diffusion and relaxation of water in blood," Magnetic Resonance in Medicine, vol. 40, pp. 79-88, July 1998.

[52] N. A. Matwiyoff, C. Gasparovic, R. Mazurchuk, and G. Matwiyoff, "The line shapes of the water proton resonances of red blood cells containing carbonyl hemoglobin, deoxyhemoglobin, and methemoglobin: Implications for the interpretation of proton MRI at fields of $1.5 \mathrm{~T}$ and below," Magnetic Resonance Imaging, vol. 8, pp. 295-301, Jan. 1990. 